\title{
Standards of Medical Care in Diabeles-2009
}

American Diabetes Association

D iabetes is a chronic illness that requires continuing medical care and patient self-management education to prevent acute complications and to reduce the risk of long-term complications. Diabetes care is complex and requires that many issues, beyond glycemic control, be addressed. A large body of evidence exists that supports a range of interventions to improve diabetes outcomes.

These standards of care are intended to provide clinicians, patients, researchers, payors, and other interested individuals with the components of diabetes care, treatment goals, and tools to evaluate the quality of care. While individual preferences, comorbidities, and other patient factors may require modification of goals, targets that are desirable for most patients with diabetes are provided. These standards are not intended to preclude more extensive evaluation and management of the patient by other specialists as needed. For more detailed information, refer to references $1-3$.

The recommendations included are screening, diagnostic, and therapeutic actions that are known or believed to favorably affect health outcomes of patients with diabetes. A grading system (Table 1), developed by the American Diabetes Association (ADA) and modeled after existing methods, was utilized to clarify and codify the evidence that forms the basis for the recommendations. The level of evidence that supports each recommendation is listed after each recommendation using the letters A, B, C, or E.

\section{CLASSIFICATION AND DIAGNOSIS}

\section{A. Classification}

In 1997, ADA issued new diagnostic and classification criteria (4); in 2003, modifications were made regarding the diagnosis of impaired fasting glucose (5). The classification of diabetes includes four clinical classes:

- type 1 diabetes (results from $\beta$-cell destruction, usually leading to absolute insulin deficiency)

- type 2 diabetes (results from a progressive insulin secretory defect on the background of insulin resistance)

- other specific types of diabetes due to other causes, e.g., genetic defects in $\beta$-cell function, genetic defects in insulin action, diseases of the exocrine pancreas (such as cystic fibrosis), and drugor chemical-induced (such as in the treatment of AIDS or after organ transplantation)

- gestational diabetes mellitus (GDM) (diabetes diagnosed during pregnancy)

Some patients cannot be clearly classified as type 1 or type 2 diabetes. Clinical presentation and disease progression vary considerably in both types of diabetes. Occasionally, patients who otherwise have type 2 diabetes may present with ketoacidosis. Similarly, patients with type 1 may have a late onset and slow (but relentless) progression of disease despite having features of autoimmune disease. Such difficulties in diagnosis may occur in children, adolescents, and adults. The true diagnosis may become more obvious over time.

The recommendations in this article are based on the evidence reviewed in the following publication: Standards of Care for Diabetes (Technical Review). Diabetes Care 17:1514-1522, 1994.

Originally approved 1988. Most recent review/revision October 2008.

DOI: $10.2337 / \mathrm{dc} 09-S 013$

C 2009 by the American Diabetes Association. Readers may use this article as long as the work is properly cited, the use is educational and not for profit, and the work is not altered. See http://creativecommons. org/licenses/by-nc-nd/3.0/ for details.

\section{B. Diagnosis of diabetes}

Current criteria for the diagnosis of diabetes in nonpregnant adults are shown in Table 2. Three ways to diagnose diabetes are recommended at the time of this statement, and each must be confirmed on a subsequent day unless unequivocal symptoms of hyperglycemia are present. Although the 75-g oral glucose tolerance test (OGTT) is more sensitive and modestly more specific than the fasting plasma glucose (FPG) to diagnose diabetes, it is poorly reproducible and difficult to perform in practice. Because of ease of use, acceptability to patients, and lower cost, the FPG has been the preferred diagnostic test. Though FPG is less sensitive than the OGTT, the vast majority of people who do not meet diagnostic criteria for diabetes by FPG but would by OGTT will have an AlC value well under 7.0\% (6).

Though the OGTT is not recommended for routine clinical use, it may be useful for further evaluation of patients in whom diabetes is still strongly suspected but who have normal FPG or IFG (see Section I.C).

The use of the AlC for the diagnosis of diabetes has previously not been recommended due to lack of global standardization and uncertainty about diagnostic thresholds. However, with a world-wide move toward a standardized assay and with increasing observational evidence about the prognostic significance of $\mathrm{AlC}$, an Expert Committee on the Diagnosis of Diabetes was convened in 2008. This joint committee of ADA, the European Association for the Study of Diabetes, and the International Diabetes Federation will likely recommend that the AlC become the preferred diagnostic test for diabetes. Diagnostic cut-points are being discussed at the time of publication of this statement. Updated recommendations will be published in Diabetes Care and will be available at diabetes.org.

\section{Diagnosis of pre-diabetes}

Hyperglycemia not sufficient to meet the diagnostic criteria for diabetes is catego- 
Table $1-A D A$ evidence grading system for clinical practice recommendations

Level of

evidence

Description

A

B

C

E

Clear evidence from well-conducted, generalizable, randomized controlled trials that are adequately powered, including:

- Evidence from a well-conducted multicenter trial

- Evidence from a meta-analysis that incorporated quality ratings in the analysis

Compelling nonexperimental evidence, i.e., "all or none" rule developed by the Centre for Evidence-Based Medicine at Oxford

Supportive evidence from well-conducted randomized controlled trials that are adequately powered, including:

- Evidence from a well-conducted trial at one or more institutions

- Evidence from a meta-analysis that incorporated quality ratings in the analysis

Supportive evidence from well-conducted cohort studies, including:

- Evidence from a well-conducted prospective cohort study or registry

- Evidence from a well-conducted meta-analysis of cohort studies

Supportive evidence from a well-conducted case-control study

Supportive evidence from poorly controlled or uncontrolled studies

- Evidence from randomized clinical trials with one or more major or three or more minor methodological flaws that could invalidate the results

- Evidence from observational studies with high potential for bias (such as case series with comparison to historical controls)

- Evidence from case series or case reports

Conflicting evidence with the weight of evidence supporting the recommendation

Expert consensus or clinical experience rized as either impaired fasting glucose (IFG) or impaired glucose tolerance (IGT), depending on whether it is identified through the FPG or the OGTT:

- $\mathrm{IFG}=\mathrm{FPG} 100 \mathrm{mg} / \mathrm{dl}(5.6 \mathrm{mmol} / \mathrm{l})$ to $125 \mathrm{mg} / \mathrm{dl}(6.9 \mathrm{mmol} / \mathrm{l})$

- $\mathrm{IGT}=2$-h plasma glucose $140 \mathrm{mg} / \mathrm{dl}$ (7.8 mmol/l) to $199 \mathrm{mg} / \mathrm{dl}(11.0$ $\mathrm{mmol} / \mathrm{l})$

IFG and IGT have been officially termed diabetes are risk factors for future diabetes and for cardiovascular disease (CVD) (7).

\section{TESTING FOR PRE- DIABETES AND DIABETES IN ASYMPTOMATIC PATIENTS}

\section{Recommendations}

- Testing to detect pre-diabetes and type 2 diabetes in asymptomatic people should be considered in adults of any age who are overweight or obese (BMI $\geq 25 \mathrm{~kg} / \mathrm{m}^{2}$ ) and who have one or more additional risk factors for diabetes ( $\mathrm{Ta}$ ble 3). In those without these risk factors, testing should begin at age 45 years. (B)

- If tests are normal, repeat testing should "pre-diabetes." Both categories of pre- be carried out at least at 3-year intervals. (E)

- To test for pre-diabetes or diabetes, an FPG test or 2-h OGTT (75-g glucose load) or both are appropriate. (B)

- An OGTT may be considered in patients with IFG to better define the risk of diabetes. (E)

- In those identified with pre-diabetes, identify and, if appropriate, treat other CVD risk factors. (B)

For many illnesses, there is a major distinction between screening and diagnostic testing. However, for diabetes, the same tests would be used for "screening" as for diagnosis. Type 2 diabetes has a long asymptomatic phase and significant clinical risk markers. Diabetes may be identified anywhere along a spectrum of clinical scenarios ranging from a seemingly low-risk individual who happens to have glucose testing, to a higher-risk individual whom the provider tests because of high suspicion of diabetes, to the symptomatic patient. The discussion herein is primarily framed as testing for diabetes in those without symptoms. Testing for diabetes will also detect individuals with prediabetes.

\section{A. Testing for pre-diabetes and type 2 diabetes in adults}

Type 2 diabetes is frequently not diagnosed until complications appear, and approximately one-third of all people with diabetes may be undiagnosed. Although the effectiveness of early identification of pre-diabetes and diabetes through mass testing of asymptomatic individuals has not been definitively proven (and rigorous trials to provide such proof are unlikely to occur), pre-diabetes and diabetes meet established criteria for conditions in which early detection is appropriate. Both conditions are common, increasing in prevalence, and impose significant public health burdens. There is a long presymptomatic phase before the diagnosis of type 2 diabetes is usually made. Relatively simple tests are available to detect preclinical disease (8). Additionally, the duration of glycemic burden is a strong predictor of adverse outcomes, and effective interventions exist to prevent progression of pre-diabetes to diabetes (see Section IV) and to reduce risk of complications of diabetes (see Section VI).

Recommendations for testing for pre-
Table 2-Criteria for the diagnosis of diabetes

\begin{tabular}{|c|c|}
\hline 1. & $\begin{array}{l}\mathrm{FPG} \geq 126 \mathrm{mg} / \mathrm{dl}(7.0 \mathrm{mmol} / \mathrm{l}) \text {. Fasting is defined as no caloric intake for at } \\
\text { least } 8 \mathrm{~h} .{ }^{*}\end{array}$ \\
\hline & OR \\
\hline 2. & $\begin{array}{l}\text { Symptoms of hyperglycemia and a casual (random) plasma glucose } \geq 200 \\
\mathrm{mg} / \mathrm{dl}(11.1 \mathrm{mmol} / \mathrm{l}) \text {. Casual (random) is defined as any time of day withou } \\
\text { regard to time since last meal. The classic symptoms of hyperglycemia } \\
\text { include polyuria, polydipsia, and unexplained weight loss. } \\
\text { OR }\end{array}$ \\
\hline 3 & $\begin{array}{l}\text { 2-h plasma glucose } \geq 200 \mathrm{mg} / \mathrm{dl}(11.1 \mathrm{mmol} / \mathrm{l}) \text { during an OGTT. The test } \\
\text { should be performed as described by the World Health Organization using } \\
\text { a glucose load containing the equivalent of } 75 \text {-g anhydrous glucose } \\
\text { dissolved in water.* }\end{array}$ \\
\hline
\end{tabular}

*In the absence of unequivocal hyperglycemia, these criteria should be confirmed by repeat testing on a different day (5). 
Table 3-Criteria for testing for pre-diabetes and diabetes in asymptomatic adult individuals

1. Testing should be considered in all adults who are overweight (BMI $\geq 25 \mathrm{~kg} / \mathrm{m}^{2 *}$ ) and have additional risk factors:

- physical inactivity

- first-degree relative with diabetes

- members of a high-risk ethnic population (e.g., African American, Latino, Native American, Asian American, Pacific Islander)

- women who delivered a baby weighing $>9$ lb or were diagnosed with GDM

- hypertension ( $\geq 140 / 90 \mathrm{mmHg}$ or on therapy for hypertension)

- HDL cholesterol level $<35 \mathrm{mg} / \mathrm{dl}(0.90 \mathrm{mmol} / \mathrm{l})$ and/or a triglyceride level $>250$ $\mathrm{mg} / \mathrm{dl}(2.82 \mathrm{mmol} / \mathrm{l})$

- women with polycystic ovarian syndrome (PCOS)

- IGT or IFG on previous testing

- other clinical conditions associated with insulin resistance (e.g., severe obesity, acanthosis nigricans)

- history of CVD

2. In the absence of the above criteria, testing for pre-diabetes and diabetes should begin at age 45 years

3. If results are normal, testing should be repeated at least at 3-year intervals, with consideration of more frequent testing depending on initial results and risk status.

*At-risk BMI may be lower in some ethnic groups.

diabetes and diabetes in asymptomatic, undiagnosed adults are listed in Table 3. Testing should be considered in adults of any age with $\mathrm{BMI} \geq 25 \mathrm{~kg} / \mathrm{m}^{2}$ and one or more risk factors for diabetes. Because age is a major risk factor for diabetes, testing of those without other risk factors should begin no later than age 45 years.

Either FPG testing or the 2-h OGTT is appropriate for testing. The 2-h OGTT identifies people with either IFG or IGT, and thus, more pre-diabetic people at increased risk for the development of diabetes and CVD. It should be noted that the two tests do not necessarily detect the same pre-diabetic individuals (9). The efficacy of interventions for primary prevention of type 2 diabetes $(10-16)$ has primarily been demonstrated among individuals with IGT, not individuals with IFG (who do not also have IGT). As noted in the diagnosis section (Section I.B), the FPG test is more convenient, more reproducible, less costly, and easier to administer than the 2-h OGTT $(4,5)$. An OGTT may be useful in patients with IFG to better define the risk of diabetes.

The appropriate interval between tests is not known (17). The rationale for the 3-year interval is that false-negatives will be repeated before substantial time elapses, and there is little likelihood that an individual will develop significant complications of diabetes within 3 years of a negative test result.

Because of the need for follow-up and discussion of abnormal results, testing should be carried out within the health care setting. Community screening outside a health care setting is not recommended because people with positive tests may not seek, or have access to, appropriate follow-up testing and care. Conversely, there may be failure to ensure appropriate repeat testing for individuals who test negative. Community screening may also be poorly targeted, i.e., it may fail to reach the groups most at risk and inappropriately test those at low risk (the worried well) or even those already diagnosed $(18,19)$.

\section{B. Testing for type 2 diabetes in children}

The incidence of type 2 diabetes in adolescents has increased dramatically in the last decade, especially in minority populations (20), although the disease remains rare in the general adolescent population (21). Consistent with recommendations for adults, children and youth at increased risk for the presence or the development of type 2 diabetes should be tested within the health care setting (22). The recommendations of the ADA consensus statement on type 2 diabetes in children and youth, with some modifications, are summarized in Table 4.

\section{Screening for type 1 diabetes}

Generally, people with type 1 diabetes present with acute symptoms of diabetes and markedly elevated blood glucose levels, and most cases are diagnosed soon after the onset of hyperglycemia. However, evidence from type 1 prevention studies suggests that measurement of islet autoantibodies identifies individuals who are at risk for developing type 1 diabetes. Such testing may be appropriate in highrisk individuals, such as those with prior transient hyperglycemia or those who have relatives with type 1 diabetes, in the context of clinical research studies (see, for example, http://www2.diabetestrialnet.org). Widespread clinical testing of asymptomatic low-risk individuals cannot currently be recommended, as it would identify very few individuals in the general population who are at risk. Individuals who screen positive should be counseled about their risk of developing diabetes. Clinical studies are being conducted to test various methods of preventing type 1 diabetes, or reversing early type 1 diabetes, in those with evidence of autoimmunity.

\section{DETECTION AND DIAGNOSIS OF GDM}

\section{Recommendations}

- Screen for GDM using risk factor analysis and, if appropriate, use of an OGTT. (C)

- Women with GDM should be screened for diabetes 6-12 weeks postpartum and should be followed up with subsequent screening for the development of diabetes or pre-diabetes. (E)

GDM is defined as any degree of glucose intolerance with onset or first recognition during pregnancy (4). Although most cases resolve with delivery, the definition applies whether or not the condition persists after pregnancy and does not exclude the possibility that unrecognized glucose intolerance may have antedated or begun concomitantly with the pregnancy. Ap-

Table 4-Testing for type 2 diabetes in asymptomatic children

Criteria:

- Overweight (BMI $>85$ th percentile for age and sex, weight for height $>85$ th percentile, or weight $>120 \%$ of ideal for height)

Plus any two of the following risk factors:

- Family history of type 2 diabetes in firstor second-degree relative

- Race/ethnicity (Native American, African American, Latino, Asian American, Pacific Islander)

- Signs of insulin resistance or conditions associated with insulin resistance (acanthosis nigricans, hypertension, dyslipidemia, PCOS, or small-forgestational-age birthweight)

- Maternal history of diabetes or GDM during the child's gestation

Age of initiation: age 10 years or at onset of puberty, if puberty occurs at a younger age

Frequency: every 3 years

Test: FPG preferred 
Table 5-Screening for and diagnosis of GDM

Carry out GDM risk assessment at the first prenatal visit.

Women at very high risk for GDM should be screened for diabetes as soon as possible after the confirmation of pregnancy. Criteria for very high risk are:

- severe obesity

- prior history of GDM or delivery of large-for-gestational-age infant

- presence of glycosuria

- diagnosis of PCOS

- strong family history of type 2 diabetes

Screening/diagnosis at this stage of pregnancy should use standard diagnostic testing (Table 2).

All women of greater than low risk of GDM, including those above not found to have diabetes early in pregnancy, should undergo GDM testing at 24-28 weeks of gestation. Low risk status, which does not require GDM screening, is defined as women with ALL of the following characteristics:

- age $<25$ years

- weight normal before pregnancy

- member of an ethnic group with a low prevalence of diabetes

- no known diabetes in first-degree relatives

- no history of abnormal glucose tolerance

- no history of poor obstetrical outcome

Two approaches may be followed for GDM screening at 24-28 weeks:

1. Two-step approach:

A. Perform initial screening by measuring plasma or serum glucose $1 \mathrm{~h}$ after a 50-g oral glucose load. A glucose threshold after 50 -g load of $\geq 140 \mathrm{mg} /$ dl identifies $\sim 80 \%$ of women with GDM, while the sensitivity is further increased to $\sim 90 \%$ by a threshold of $\geq 130 \mathrm{mg} / \mathrm{dl}$.

B. Perform a diagnostic 100-g OGTT on a separate day in women who exceed the chosen threshold on 50 -g screening.

2. One-step approach (may be preferred in clinics with high prevalence of GDM): Perform a diagnostic 100-g OGTT in all women to be tested at 24-28 weeks.

The 100-g OGTT should be performed in the morning after an overnight fast of at least $8 \mathrm{~h}$. To make a diagnosis of GDM, at least two of the following plasma glucose values must be found:

Fasting: $\geq 95 \mathrm{mg} / \mathrm{dl}$

$1 \mathrm{~h}: \geq 180 \mathrm{mg} / \mathrm{dl}$

$2 \mathrm{~h}: \geq 155 \mathrm{mg} / \mathrm{dl}$

$3 \mathrm{~h}: \geq 140 \mathrm{mg} / \mathrm{dl}$

proximately $7 \%$ of all pregnancies (ranging from 1 to $14 \%$ depending on the population studied and the diagnostic tests employed) are complicated by GDM, resulting in more than 200,000 cases annually.

Because of the risks of GDM to the mother and neonate, screening and diagnosis are warranted. The screening and diagnostic strategies, based on the 2004 ADA position statement on gestational diabetes mellitus (23), are outlined in Table 5.

Results of the Hyperglycemia and Adverse Pregnancy Outcomes study (24), a large-scale (including 25,000 pregnant women) multinational epidemiologic study, demonstrated that risk of adverse maternal, fetal, and neonatal outcomes continuously increased as a function of maternal glycemia at 24-28 weeks, even within ranges previously considered normal for pregnancy. For most complications, there was no threshold for risk.
These results have led to careful reconsideration of the diagnostic criteria for GDM. An international group representing multiple obstetrical and diabetes organizations, including ADA, is currently working on consensus toward 1) a worldwide standard for which diagnostic test to use for GDM and 2) rational diagnostic cut points.

Because women with a history of GDM have a greatly increased subsequent risk for diabetes (25), they should be screened for diabetes 6-12 weeks postpartum, using nonpregnant OGTT criteria, and should be followed up with subsequent screening for the development of diabetes or pre-diabetes, as outlined in Section II. For information on the National Diabetes Education Program (NDEP) campaign to prevent type 2 diabetes in women with GDM, go to www.ndep.nih.gov/diabetes/pubs/ NeverTooEarly_Tipsheet.pdf.

\section{PREVENTION/DELAY OF TYPE 2 DIABETES}

\section{Recommendations}

- Patients with IGT (A) or IFG (E) should be referred to an effective ongoing support program for weight loss of 5-10\% of body weight and for increasing physical activity to at least 150 min per week of moderate activity such as walking.

- Follow-up counseling appears to be important for success. (B)

- Based on potential cost savings of diabetes prevention, such counseling should be covered by third-party payors. (E)

- In addition to lifestyle counseling, metformin may be considered in those who are at very high risk for developing diabetes (combined IFG and IGT plus other risk factors such as $\mathrm{AlC}>6 \%$, hypertension, low HDL cholesterol, elevated triglycerides, or family history of diabetes in a first-degree relative) and who are obese and under 60 years of age. (E)

- Monitoring for the development of diabetes in those with pre-diabetes should be performed every year. (E)

Randomized controlled trials have shown that individuals at high risk for developing diabetes (those with IFG, IGT, or both) can be given interventions that significantly decrease the rate of onset of diabetes (10-16). These interventions include intensive lifestyle modification programs that have been shown to be very effective ( $\geq 58 \%$ reduction after 3 years) and use of the pharmacologic agents metformin, acarbose, orlistat, and thiazolidinediones (TZDs), each of which has been shown to decrease incident diabetes to various degrees. A summary of major diabetes prevention trials is shown in Table 6 .

Two studies of lifestyle intervention have shown persistent reduction in the rate of conversion to type 2 diabetes with 3 (26) to 14 years (27) of postintervention follow-up.

Based on the results of clinical trials and the known risks of progression of pre-diabetes to diabetes, an ADA Consensus Development Panel (7) concluded that persons with pre-diabetes (IGT and/or IFG) should be counseled on lifestyle changes with goals similar to those of the Diabetes Prevention Program (DPP) (5-10\% weight loss and moderate physical activity of $\sim 30$ min per day). Regarding the more difficult issue of drug 


\begin{tabular}{|c|c|c|c|c|c|c|c|}
\hline Study (ref.) & $n$ & Population & $\begin{array}{c}\text { Mean } \\
\text { age } \\
\text { (years) }\end{array}$ & $\begin{array}{l}\text { Duration } \\
\text { (years) }\end{array}$ & $\begin{array}{c}\text { Intervention } \\
\text { (daily dose) }\end{array}$ & $\begin{array}{c}\text { Conversion in } \\
\text { control subjects } \\
(\% / \text { year })\end{array}$ & Relative risk \\
\hline \multicolumn{8}{|l|}{ Lifestyle } \\
\hline Finnish DPS (11) & 522 & IGT, BMI $\geq 25 \mathrm{~kg} / \mathrm{m}^{2}$ & 55 & 3.2 & $\begin{array}{l}\text { Individual } \\
\text { diet/exercise }\end{array}$ & 6 & $0.42(0.30-0.70)$ \\
\hline $\operatorname{DPP}(10)$ & $2,161^{*}$ & $\begin{array}{l}\mathrm{IGT}, \mathrm{BMI} \geq 24 \mathrm{~kg} / \mathrm{m}^{2} \\
\mathrm{FPG}>5.3 \mathrm{mmol} / \mathrm{l}\end{array}$ & 51 & 3 & $\begin{array}{l}\text { Individual } \\
\text { diet/exercise }\end{array}$ & 10 & $0.42(0.34-0.52)$ \\
\hline Da Qing (12) & $259 *$ & $\begin{array}{l}\text { IGT (randomized } \\
\text { groups) }\end{array}$ & 45 & 6 & Group diet/exercise & 16 & $0.62(0.44-0.86)$ \\
\hline Toranomon study (28) & 458 & $\begin{array}{l}\text { IGT (men), BMI }=24 \\
\mathrm{~kg} / \mathrm{m}^{2}\end{array}$ & 55 & 4 & $\begin{array}{l}\text { Individual } \\
\text { diet/exercise }\end{array}$ & 2 & $0.33(0.10-1.0) \dagger$ \\
\hline Indian DPP (16) & $269^{*}$ & IGT & 46 & 2.5 & $\begin{array}{l}\text { Individual } \\
\text { diet/exercise }\end{array}$ & 22 & $0.71(0.63-0.79)$ \\
\hline \multicolumn{8}{|l|}{ Medications } \\
\hline $\operatorname{DPP}(10)$ & $2,155^{*}$ & $\begin{array}{l}\mathrm{IGT}, \mathrm{BMI}>24 \mathrm{~kg} / \mathrm{m}^{2} \\
\quad \mathrm{FPG}>5.3 \mathrm{mmol} / \mathrm{l}\end{array}$ & 51 & 2.8 & Metformin (1,700 mg) & 10 & $0.69(0.57-0.83)$ \\
\hline Indian DPP (16) & $269 *$ & IGT & 46 & 2.5 & Metformin (500 mg) & 22 & $0.74(0.65-0.81)$ \\
\hline STOP NIDDM (14) & 1,419 & IGT, FPG $>5.6 \mathrm{mmol} / \mathrm{l}$ & 54 & 3.2 & Acarbose (300 mg) & 13 & $0.75(0.63-0.90)$ \\
\hline XENDOS (29) & 3,277 & $\mathrm{BMI}>30 \mathrm{~kg} / \mathrm{m}^{2}$ & 43 & 4 & Orlistat (360 mg) & 2 & $0.63(0.46-0.86)$ \\
\hline DREAM (15) & 5,269 & IGT or IFG & 55 & 3.0 & Rosiglitazone (8 mg) & 9 & $0.40(0.35-0.46)$ \\
\hline
\end{tabular}

*Number of participants in the indicated comparisons, not necessarily in the entire study. †Calculated from information in the article. DPP, Diabetes Prevention Program; DREAM, Diabetes REduction Assessment with ramipril and rosiglitazone Medication; DPS, Diabetes Prevention Study; STOP NIDDM, Study to Prevent Non-Insulin Dependent Diabetes; XENDOS, Xenical in the prevention of Diabetes in Obese Subjects. This table has been reprinted with permission (30) with some modification.

therapy for diabetes prevention, the consensus panel felt that metformin should be the only drug considered for use in diabetes prevention. For other drugs, the issues of cost, side effects, and lack of persistence of effect in some studies led the panel to not recommend their use for diabetes prevention. Metformin use was recommended only for very-high-risk individuals (those with combined IGT and IFG who are obese and under 60 years of age with at least one other risk factor for diabetes). In addition, the panel highlighted the evidence that in the DPP, metformin was most effective compared to lifestyle in those with BMI of at least 35 $\mathrm{kg} / \mathrm{m}^{2}$ and those under age 60 years.

\section{DIABETES CARE}

\section{A. Initial evaluation}

A complete medical evaluation should be performed to classify the diabetes, detect the presence of diabetes complications, review previous treatment and glycemic control in patients with established diabetes, assist in formulating a management plan, and provide a basis for continuing care. Laboratory tests appropriate to the evaluation of each patient's medical condition should be performed. A focus on the components of comprehensive care (Table 7) will assist the health care team to ensure optimal management of the patient with diabetes.

\section{B. Management}

People with diabetes should receive medical care from a physician-coordinated team. Such teams may include, but are not limited to, physicians, nurse practitioners, physician's assistants, nurses, dietitians, pharmacists, and mental health professionals with expertise and a special interest in diabetes. It is essential in this collaborative and integrated team approach that individuals with diabetes assume an active role in their care.

The management plan should be formulated as an individualized therapeutic alliance among the patient and family, the physician, and other members of the health care team. A variety of strategies and techniques should be used to provide adequate education and development of problem-solving skills in the various aspects of diabetes management. Implementation of the management plan requires that each aspect is understood and agreed on by the patient and the care providers and that the goals and treatment plan are reasonable. Any plan should recognize diabetes self-management education (DSME) as an integral component of care. In developing the plan, consideration should be given to the patient's age, school or work schedule and conditions, physical activity, eating patterns, social situation and personality, cultural factors, and presence of complications of diabetes or other medical conditions.

\section{Glycemic control}

1. Assessment of glycemic control

Two primary techniques are available for health providers and patients to assess the effectiveness of the management plan on glycemic control: patient self-monitoring of blood glucose (SMBG) or of interstitial glucose and measurement of AlC.

\section{a. Glucose monitoring}

\section{Recommendations}

- SMBG should be carried out three or more times daily for patients using multiple insulin injections or insulin pump therapy. (A)

- For patients using less frequent insulin injections, noninsulin therapies, or medical nutrition therapy (MNT) and physical activity alone, SMBG may be useful as a guide to the success of therapy. (E)

- To achieve postprandial glucose targets, postprandial SMBG may be appropriate. (E) 


\section{Table 7-Components of the comprehensive diabetes evaluation}

Medical history

- age and characteristics of onset of diabetes (e.g., DKA, asymptomatic laboratory finding)

- eating patterns, physical activity habits, nutritional status, and weight history; growth and development in children and adolescents

- diabetes education history

- review of previous treatment regimens and response to therapy (AlC records)

- current treatment of diabetes, including medications, meal plan, physical activity patterns, and results of glucose monitoring and patient's use of data

- DKA frequency, severity, and cause

- hypoglycemic episodes

- hypoglycemia awareness

- any severe hypoglycemia: frequency and cause

- history of diabetes-related complications

- microvascular: retinopathy, nephropathy, neuropathy (sensory, including history of foot lesions; autonomic, including sexual dysfunction and gastroparesis)

- macrovascular: CHD, cerebrovascular disease, PAD

- other: psychosocial problems, ${ }^{*}$ dental disease*

Physical examination

- height, weight, BMI

- blood pressure determination, including orthostatic measurements when indicated

- fundoscopic examination*

- thyroid palpation

- skin examination (for acanthosis nigricans and insulin injection sites)

- comprehensive foot examination:

- inspection

- palpation of dorsalis pedis and posterior tibial pulses

- presence/absence of patellar and Achilles reflexes

- determination of proprioception, vibration, and monofilament sensation

Laboratory evaluation

- A1C, if results not available within past 2-3 months

If not performed/available within past year:

- fasting lipid profile, including total, LDL- and HDL-cholesterol and triglycerides

- liver function tests

- test for urine albumin excretion with spot urine albumin/creatinine ratio

- serum creatinine and calculated GFR

- thyroid-stimulating hormone in type 1 diabetes, dyslipidemia or women over age 50 Referrals

- annual dilated eye exam

- family planning for women of reproductive age

- registered dietitian for MNT

- diabetes self-management education

- dental examination

- mental Health professional, if needed

*See appropriate referrals for these categories.

- When prescribing SMBG, ensure that patients receive initial instruction in, and routine follow-up evaluation of, SMBG technique and their ability to use data to adjust therapy. (E)

- Continuous glucose monitoring (CGM) in conjunction with intensive insulin regimens can be a useful tool to lower AlC in selected adults (age $\geq 25$ years) with type 1 diabetes (A).

- Although the evidence for AlC lowering is less strong in children, teens, and younger adults, CGM may be helpful in these groups. Success correlates with adherence to ongoing use of the device. (C)
- CGM may be a supplemental tool to SMBG in those with hypoglycemia unawareness and/or frequent hypoglycemic episodes. (E)

The ADA's consensus and position statements on SMBG provide a comprehensive review of the subject $(31,32)$. Major clinical trials of insulin-treated patients that demonstrated the benefits of intensive glycemic control on diabetes complications have included SMBG as part of multifactorial interventions, suggesting that SMBG is a component of effective therapy. SMBG allows patients to evaluate their individual response to therapy and assess whether glycemic targets are being achieved. Results of SMBG can be useful in preventing hypoglycemia and adjusting medications (particularly prandial insulin doses), MNT, and physical activity.

The frequency and timing of SMBG should be dictated by the particular needs and goals of the patients. SMBG is especially important for patients treated with insulin to monitor for and prevent asymptomatic hypoglycemia and hyperglycemia. For most patients with type 1 diabetes and pregnant women taking insulin, SMBG is recommended three or more times daily. For this population, significantly more frequent testing may be required to reach AlC targets safely without hypoglycemia. The optimal frequency and timing of SMBG for patients with type 2 diabetes on noninsulin therapy is unclear. A meta-analysis of SMBG in noninsulin-treated patients with type 2 diabetes concluded that some regimen of SMBG was associated with a reduction in $\mathrm{AlC}$ of $\geq 0.4 \%$. However, many of the studies in this analysis also included patient education with diet and exercise counseling and, in some cases, pharmacologic intervention, making it difficult to assess the contribution of SMBG alone to improved control (33). Several recent trials have called into question the clinical utility and cost-effectiveness of routine SMBG in non-insulin-treated patients (34-36).

Because the accuracy of SMBG is instrument and user dependent (37), it is important to evaluate each patient's monitoring technique, both initially and at regular intervals thereafter. In addition, optimal use of SMBG requires proper interpretation of the data. Patients should be taught how to use the data to adjust food intake, exercise, or pharmacological therapy to achieve specific glycemic goals, and these skills should be reevaluated periodically.

CGM through the measurement of interstitial glucose (which correlates well with plasma glucose) is available. These sensors require calibration with SMBG, and the latter are still recommended for making acute treatment decisions. CGM devices also have alarms for hypo- and hyperglycemic excursions. Small studies in selected patients with type 1 diabetes have suggested that CGM use reduces the time spent in hypo- and hyperglycemic ranges and may modestly improve glycemic control. A larger 26-week randomized trial of 322 type 1 patients showed that adults age 25 years and older using 
intensive insulin therapy and CGM experienced a $0.5 \%$ reduction in $\mathrm{AlC}$ (from $\sim 7.6$ to $7.1 \%$ ) compared with usual intensive insulin therapy with SMBG (38). Sensor use in children, teens, and adults to age 24 years did not result in significant AlC lowering, and there was no significant difference in hypoglycemia in any group. Importantly, the greatest predictor of AlC lowering in this study for all agegroups was frequency of sensor use, which was lower in younger age-groups. Although CGM is an evolving technology, emerging data suggest that, in appropriately selected patients who are motivated to wear it most of the time, it may offer benefit. CGM may be particularly useful in those with hypoglycemia unawareness and/or frequent episodes of hypoglycemia, and studies in this area are ongoing.

\section{b. A1C}

\section{Recommendations}

- Perform the AlC test at least two times a year in patients who are meeting treatment goals (and who have stable glycemic control). (E)

- Perform the AlC test quarterly in patients whose therapy has changed or who are not meeting glycemic goals. (E)

- Use of point-of-care testing for AlC allows for timely decisions on therapy changes, when needed. (E)

Because $\mathrm{AlC}$ is thought to reflect average glycemia over several months (37), and has strong predictive value for diabetes complications $(10,39)$, AlC testing should be performed routinely in all patients with diabetes at initial assessment and then as part of continuing care. Measurement approximately every 3 months determines whether a patient's glycemic targets have been reached and maintained. For any individual patient, the frequency of AlC testing should be dependent on the clinical situation, the treatment regimen used, and the judgment of the clinician. Some patients with stable glycemia well within target may do well with testing only twice per year, while unstable or highly intensively managed patients (e.g., pregnant type 1 women) may be tested more frequently than every 3 months. The availability of the $\mathrm{AlC}$ result at the time that the patient is seen (point-of-care testing) has been reported to result in increased intensification of therapy and improvement in glycemic control $(40,41)$.

The AlC test is subject to certain lim-
Table 8-Correlation of A1C with average glucose

\begin{tabular}{lcc} 
& \multicolumn{2}{c}{ Mean plasma glucose } \\
\cline { 2 - 3 } AlC (\%) & $\mathrm{mg} / \mathrm{dl}$ & $\mathrm{mmol} / \mathrm{l}$ \\
\hline 6 & 126 & 7.0 \\
7 & 154 & 8.6 \\
8 & 183 & 10.2 \\
9 & 212 & 11.8 \\
10 & 240 & 13.4 \\
11 & 269 & 14.9 \\
12 & 298 & 16.5 \\
\hline
\end{tabular}

Estimates based on ADAG data of $\sim 2,700$ glucose measurements over 3 months per AlC measurement in 507 adults with type 1 , type 2 , and no diabetes. Correlation between $\mathrm{AlC}$ and average glucose: 0.92 (42). A calculator for converting AlC results into eAG, in either $\mathrm{mg} / \mathrm{dl}$ or $\mathrm{mmol} / \mathrm{l}$, is available at http://professional.diabetes.org/eAG.

itations. Conditions that affect erythrocyte turnover (hemolysis, blood loss) and hemoglobin variants must be considered, particularly when the AlC result does not correlate with the patient's clinical situation (37). In addition, AlC does not provide a measure of glycemic variability or hypoglycemia. For patients prone to glycemic variability (especially type 1 patients, or type 2 patients with severe insulin deficiency), glycemic control is best judged by the combination of results of SMBG testing and the AlC. The AlC may also serve as a check on the accuracy of the patient's meter (or the patient's reported SMBG results) and the adequacy of the SMBG testing schedule.

Table 8 contains the correlation between AlC levels and mean plasma glucose levels based on data from the international AlC-Derived Average Glucose (ADAG) trial utilizing frequent SMBG and continuous glucose monitoring in 507 adults (83\% Caucasian) with type 1 , type 2 , and no diabetes (49) The ADA and American Association of Clinical Chemists have determined that the correlation ( $r=0.92)$ is strong enough to justify reporting both an $\mathrm{AlC}$ result and an estimated average glucose (eAG) result when a clinician orders the AlC test. The table in previous versions of the Standards of Medical Care in Diabetes describing the correlation between AlC and mean glucose was derived from relatively sparse data (one seven-point profile over 1 day per $\mathrm{AlC}$ reading) in the primarily Caucasian type 1 participants in the Diabetes Control and Complications Trial (DCCT) trial (43). Clinicians should note that the numbers in the table are now dif- ferent, as they are based on $\sim 2,800$ readings per $\mathrm{AlC}$ in the $\mathrm{ADAG}$ trial.

In the ADAG study, there were no significant differences among racial and ethnic groups in the regression lines between $\mathrm{AlC}$ and mean glucose, although there was a trend toward a difference between African/African-American and Caucasian participants' regression lines that might have been significant had more African/ African-American participants been studied. A recent study comparing $\mathrm{AlC}$ to CGM data in 48 type 1 children found a highly statistically significant correlation between A1C and mean blood glucose, although the correlation $(r=0.7)$ was significantly lower than in the ADAG trial (44). Whether there are significant differences in how $\mathrm{AlC}$ relates to average glucose in children or in African-American patients is an area for further study. For the time being, the question has not led to different recommendations about testing $\mathrm{AlC}$ or to different interpretations of the clinical meaning of given levels of AlC in those populations.

For patients in whom AlC/eAG and measured blood glucose appear discrepant, clinicians should consider the possibilities of hemoglobinopathy or altered red cell turnover and the options of more frequent and/or different timing of SMBG or use of CGM. Other measures of chronic glycemia such as fructosamine are available, but their linkage to average glucose and their prognostic significance are not as clear as is the case for AlC.

\section{Glycemic goals in adults}

- Lowering A1C to below or around 7\% has been shown to reduce microvascular and neuropathic complications of type 1 and type 2 diabetes. Therefore, for microvascular disease prevention, the AlC goal for nonpregnant adults in general is $<7 \%$. (A)

- In type 1 and type 2 diabetes, randomized controlled trials of intensive versus standard glycemic control have not shown a significant reduction in CVD outcomes during the randomized portion of the trials. Long-term follow-up of the DCCT and UK Prospective Diabetes Study (UKPDS) cohorts suggests that treatment to AlC targets below or around $7 \%$ in the years soon after the diagnosis of diabetes is associated with long-term reduction in risk of macrovascular disease. Until more evidence becomes available, the general goal of $<7 \%$ appears reasonable for many 
adults for macrovascular risk reduction. (B)

- Subgroup analyses of clinical trials such as the DCCT and UKPDS and the microvascular evidence from the Action in Diabetes and Vascular Disease: Preterax and Diamicron MR Controlled Evaluation (ADVANCE) trial suggest a small but incremental benefit in microvascular outcomes with $\mathrm{AlC}$ values closer to normal. Therefore, for selected individual patients, providers might reasonably suggest even lower AlC goals than the general goal of $<7 \%$, if this can be achieved without significant hypoglycemia or other adverse effects of treatment. Such patients might include those with short duration of diabetes, long life expectancy, and no significant CVD. (B)

- Conversely, less stringent AlC goals than the general goal of $<7 \%$ may be appropriate for patients with a history of severe hypoglycemia, limited life expectancy, advanced microvascular or macrovascular complications, extensive comorbid conditions, and those with longstanding diabetes in whom the general goal is difficult to attain despite DSME, appropriate glucose monitoring, and effective doses of multiple glucose-lowering agents including insulin. (C)

Glycemic control is fundamental to the management of diabetes. The DCCT, a prospective, randomized, controlled trial of intensive versus standard glycemic control in patients with relatively recently diagnosed type 1 diabetes, showed definitively that improved glycemic control is associated with significantly decreased rates of microvascular (retinopathy and nephropathy) as well as neuropathic complications (45). Follow-up of the DCCT cohorts in the Epidemiology of Diabetes Interventions and Complications (EDIC) study has shown persistence of this effect in previously intensively treated subjects, even though their glycemic control has been equivalent to that of previous standard arm subjects during follow-up $(46,47)$.

In type 2 diabetes, the Kumamoto study $(48)$ and the UKPDS $(49,50)$ demonstrated significant reductions in microvascular and neuropathic complications with intensive therapy. Similar to the DCCT-EDIC findings, long-term follow-up of the UKPDS cohort has recently demonstrated a "legacy effect" of early intensive glycemic control on long-term rates of microvascular complications, even with loss of glycemic separation between the intensive and standard cohorts after the end of the randomized controlled (51).

In each of these large randomized prospective clinical trials, treatment regimens that reduced average AlC to $\geq 7 \%$ ( $\geq 1 \%$ above the upper limits of normal) were associated with fewer long-term microvascular complications; however, intensive control was found to increase the risk of severe hypoglycemia, most notably in the DCCT, and led to weight gain $(39,52)$.

Epidemiological analyses of the DCCT and UKPDS $(39,45)$ demonstrate a curvilinear relationship between AlC and microvascular complications. Such analyses suggest that, on a population level, the greatest number of complications will be averted by taking patients from very poor control to fair or good control. These analyses also suggest that further lowering of AlC from 7 to $6 \%$ is associated with further reduction in the risk of microvascular complications, albeit the absolute risk reductions become much smaller. Given the substantially increased risk of hypoglycemia (particularly in those with type 1 diabetes) and the relatively much greater effort required to achieve nearnormoglycemia, the risks of lower targets may outweigh the potential benefits on microvascular complications on a population level. However, selected individual patients, especially those with little comorbidity and long life expectancy (who may reap the benefits of further lowering of glycemia below 7\%) may, at patient and provider judgment, adopt glycemic targets as close to normal as possible as long as significant hypoglycemia does not become a barrier.

Whereas many epidemiologic studies and meta-analyses $(53,54)$ have clearly shown a direct relationship between AlC and CVD, the potential of intensive glycemic control to reduce CVD has been less clearly defined. In the DCCT, there was a trend toward lower risk of CVD events with intensive control (risk reduction $41 \%, 95 \%$ CI 10-68\%), but the number of events was small. However, 9-year post-DCCT follow-up of the cohort has shown that participants previously randomized to the intensive arm had a $42 \%$ reduction $(P=0.02)$ in CVD outcomes and a $57 \%$ reduction $(P=0.02)$ in the risk of nonfatal myocardial infarction (MI), stroke, or CVD death compared with those previously in the standard arm (55).

The UKPDS trial of type 2 diabetes observed a $16 \%$ reduction in cardiovascular complications (combined fatal or nonfatal MI and sudden death) in the intensive glycemic control arm, although this difference was not statistically significant $(P=0.052)$, and there was no suggestion of benefit on other CVD outcomes such as stroke. In an epidemiologic analysis of the study cohort, a continuous association was observed, such that for every percentage point lower median onstudy AlC (e.g., 8 to $7 \%$ ) there was a statistically significant $18 \%$ reduction in CVD events, again with no glycemic threshold. A recent report of 10 years of follow-up of the UKPDS cohort describes, for the participants originally randomized to intensive glycemic control compared with those randomized to conventional glycemic control, long-term reductions in MI (15\% with sulfonylurea or insulin as initial pharmacotherapy, 33\% with metformin as initial pharmacotherapy, both statistically significant) and in all-cause mortality (13 and 27\%, respectively, both statistically significant) (51).

Because of ongoing uncertainty regarding whether intensive glycemic control can reduce the increased risk of CVD events in people with type 2 diabetes, several large long-term trials were launched in the past decade to compare the effects of intensive versus standard glycemic control on CVD outcomes in relatively high-risk participants with established type 2 diabetes.

The Action to Control Cardiovascular Risk in Diabetes (ACCORD) study randomized 10,251 participants with either history of a CVD event (ages $40-79$ years) or significant CVD risk (ages 55-79) to a strategy of intensive glycemic control (target AlC $<6.0 \%$ ) or standard glycemic control (AlC target 7.0-7.9\%). Investigators used multiple glycemic medications in both arms. ACCORD participants were on average 62 years old and had a mean duration of diabetes of 10 years, with 35\% already treated with insulin at baseline. From a baseline median AlC of $8.1 \%$, the intensive arm reached a median AlC of $6.4 \%$ within 12 months of randomization, while the standard group reached a median AlC of $7.5 \%$. Other risk factors were treated aggressively and equally in both groups. The intensive glycemic control group had more use of insulin in combination with multiple oral agents, significantly more weight gain, and more 
episodes of severe hypoglycemia than the standard group.

In February 2008, the glycemic control study of ACCORD was halted on the recommendation of the study's data safety monitoring board due to the finding of an increased rate of mortality in the intensive arm compared with the standard arm (1.41\%/year vs. $1.14 \%$ /year; HR 1.22 [95\% CI 1.01-1.46]), with a similar increase in cardiovascular deaths. The primary outcome of ACCORD (MI, stroke, or cardiovascular death) was lower in the intensive glycemic control group due to a reduction in nonfatal MI, although this finding was not statistically significant when the study was terminated (HR 0.90 [95\% CI 0.78-1.04]; $P=0.16$ ) (56).

Exploratory analyses of the mortality findings of ACCORD (evaluating variables including weight gain, use of any specific drug or drug combination, and hypoglycemia) were reportedly unable to identify an explanation for the excess mortality in the intensive arm. Prespecified subset analyses showed that participants with no previous CVD event and those who had a baseline AlC $<8 \%$ had a statistically significant reduction in the primary CVD outcome.

The ADVANCE study randomized 11,140 participants to a strategy of intensive glycemic control (with primary therapy being the sulfonylurea gliclizide and additional medications as needed to achieve a target $\mathrm{AlC}$ of $\leq 6.5 \%$ ) or to standard therapy (in which any medication but gliclizide could be used and the glycemic target was according to "local guidelines"). ADVANCE participants (who had to be at least 55 years of age with either known vascular disease or at least one other vascular risk factor) were slightly older and of similar high CVD risk as those in ACCORD. However, they had an average duration of diabetes 2 years shorter, lower baseline AlC (median $7.2 \%$ ), and almost no use of insulin at enrollment. The median AlC levels achieved in the intensive and standard arms were 6.3 and $7.0 \%$, respectively, and maximal separation between the arms took several years to achieve. Use of other drugs that favorably impact CVD risk (aspirin, statins, ACE inhibitors) was lower in ADVANCE than in the ACCORD or Veterans Affairs Diabetes Trial (VADT).

The primary outcome of ADVANCE was a combination of microvascular events (nephropathy and retinopathy) and major adverse cardiovascular events (MI, stroke, and cardiovascular death).
Intensive glycemic control significantly reduced the primary endpoint (HR 0.90 [95\% CI 0.82-0.98]; $P=0.01$ ), although this was due to a significant reduction in the microvascular outcome (0.86 [0.77$0.97], P=0.01$ ), primarily development of macroalbuminuria, with no significant reduction in the macrovascular outcome (0.94 [0.84-1.06]; $P=0.32$ ). There was no difference in overall or cardiovascular mortality between the intensive and the standard glycemic control arms (57).

The VADT randomized 1,791 participants with type 2 diabetes uncontrolled on insulin or maximal dose oral agents (median entry AlC 9.4\%) to a strategy of intensive glycemic control (goal AlC $<6.0 \%$ ) or standard glycemic control, with a planned AlC separation of at least $1.5 \%$. Medication treatment algorithms were used to achieve the specified glycemic goals, with a goal of using similar medications in both groups. Median AlC levels of 6.9 and $8.4 \%$ were achieved in the intensive and standard arms, respectively, within the first year of the study. Other CVD risk factors were treated aggressively and equally in both groups.

The primary outcome of the VADT was a composite of CVD events (MI, stroke, cardiovascular death, revascularization, hospitalization for heart failure, and amputation for ischemia). During a mean 6-year follow-up period, the cumulative primary outcome was nonsignificantly lower in the intensive arm (HR 0.87 [95\% CI 0.73-1.04]; $P=0.12$ ). There were more CVD deaths in the intensive arm than in the standard arm (40 vs. 33; sudden deaths 11 vs. 4 ), but the difference was not statistically significant. Post hoc subgroup analyses suggested that duration of diabetes interacted with randomization such that participants with duration of diabetes less than about 12 years appeared to have a CVD benefit of intensive glycemic control while those with longer duration of disease before study entry had a neutral or even adverse effect of intensive glycemic control. Other exploratory analyses suggested that severe hypoglycemia within the past 90 days was a strong predictor of the primary outcome and of CVD mortality (58).

The cause of the excess deaths in the intensive glycemic control arm of ACCORD compared with the standard arm has been difficult to pinpoint. By design of the trial, randomization to the intensive arm was associated with or led to many downstream effects, such as higher rates of severe hypoglycemia; more frequent use of insulin, TZDs, other drugs, and drug combinations; and greater weight gain. Such factors may be associated statistically with the higher mortality rate in the intensive arm but may not be causative. It is biologically plausible that severe hypoglycemia could increase the risk of cardiovascular death in participants with high underlying CVD risk. Other plausible mechanisms for the increase in mortality in ACCORD include weight gain, unmeasured drug effects or interactions, or the overall "intensity" of the ACCORD intervention (use of multiple oral glucose-lowering drugs along with multiple doses of insulin, frequent therapy adjustments to push AlC and selfmonitored blood glucose to very low targets, and an intense effort to aggressively reduce AlC by $\sim 2 \%$ in participants entering the trial with advanced diabetes and multiple comorbidities).

Since the ADVANCE trial did not show any increase in mortality in the intensive glycemic control arm, examining the differences between ADVANCE and ACCORD supports additional hypotheses. ADVANCE participants on average appeared to have earlier or less advanced diabetes, with shorter duration by $2-3$ years and lower AlC at entry despite very little use of insulin at baseline. AlC was also lowered less and more gradually in the ADVANCE trial, and there was no significant weight gain with intensive glycemic therapy. Although severe hypoglycemia was defined somewhat differently in the three trials, it appears that this occurred in fewer than 3\% of intensively treated ADVANCE participants for the entire study duration (median 5 years) compared with $\sim 16 \%$ of intensively treated subjects in ACCORD and $21 \%$ in VADT

It is likely that the increase in mortality in ACCORD was related to the overall treatment strategies for intensifying glycemic control in the study population, not the achieved AlC per se. The ADVANCE study achieved a median AlC in its intensive arm similar to that in the ACCORD study, with no increased mortality hazard. Thus, the ACCORD mortality findings do not imply that patients with type 2 diabetes who can easily achieve or maintain low AlC levels with lifestyle modifications with or without pharmacotherapy are at risk and need to "raise" their AlC.

The three trials compared treatments to AlC levels in the "flatter" part of the observational glycemia-CVD risk curves (median AlC of $6.4-6.9 \%$ in the inten- 
Table 9-Summary of glycemic recommendations for non-pregnant adults with diabetes

AlC

Preprandial capillary plasma glucose

Peak postprandial capillary plasma glucose

Key concepts in setting glycemic goals:

- $\mathrm{AlC}$ is the primary target for glycemic control.

- Goals should be individualized based on:

- duration of diabetes

- age/life expectancy

- comorbid conditions

- known CVD or advanced microvascular complications

- hypoglycemia unawareness

- individual patient considerations

- More or less stringent glycemic goals may be appropriate for individual patients.

- Postprandial glucose may be targeted if AlC goals are not met despite reaching preprandial glucose goals.

*Referenced to a nondiabetic range of $4.0-6.0 \%$ using a DCCT-based assay. Postprandial glucose measurements should be made $1-2 \mathrm{~h}$ after the beginning of the meal, generally peak levels in patients with diabetes.

sive arms compared with $7.0-8.4 \%$ in the standard arms). Importantly, their results should not be extrapolated to imply that there would be no cardiovascular benefit of glucose lowering from very poor control (e.g., AlC >9\%) to good control (e.g., AlC <7\%).

All three trials were carried out in participants with established diabetes (mean duration 8-11 years) and either known CVD or multiple risk factors suggesting the presence of established atherosclerosis. Subset analyses of the three trials suggested a significant benefit of intensive glycemic control on CVD in participants with shorter duration of diabetes, lower $\mathrm{AlC}$ at entry, and/or or absence of known CVD. The DCCT-EDIC study and the long-term follow-up of the UKPDS cohort both suggest that intensive glycemic control initiated soon after diagnosis of diabetes in patients with a lower level of CVD risk may impart long-term protection from CVD events. As is the case with microvascular complications, it may be that glycemic control plays a greater role before macrovascular disease is well developed and minimal or no role when it is advanced.

The benefits of intensive glycemic control on microvascular and neuropathic complications are well established for both type 1 and type 2 diabetes. The ADVANCE trial has added to that evidence base by demonstrating a significant reduction in the risk of new or worsening albuminuria when $\mathrm{AlC}$ was lowered to 6.3\% compared with standard glycemic control achieving an AlC of $7.0 \%$. The lack of significant reduction in CVD events with intensive glycemic control in ACCORD, ADVANCE, and VADT should not lead clinicians to abandon the general target of an AlC $<7.0 \%$ and thereby discount the benefit of good control on what are serious and debilitating microvascular complications.

The evidence for a cardiovascular benefit of intensive glycemic control primarily rests on long-term follow-up of study cohorts treated early in the course of type 1 and type 2 diabetes and subset analyses of ACCORD, ADVANCE, and VADT. Conversely, the mortality findings in ACCORD suggest that the potential risks of very intensive glycemic control may outweigh its benefits in some patients, such as those with very long duration of diabetes, known history of severe hypoglycemia, advanced atherosclerosis, and advanced age/frailty. Certainly, providers should be vigilant in preventing severe hypoglycemia in patients with advanced disease and should not aggressively attempt to achieve near-normal AlC levels in patients in whom such a target cannot be reasonably easily and safely achieved.

Recommended glycemic goals for nonpregnant adults are shown in Table 9. The recommendations are based on those for AlC, with listed blood glucose levels that appear to correlate with achievement of an AlC of $<7 \%$. The issue of pre- versus postprandial SMBG targets is complex (59). Elevated postchallenge (2-h OGTT) glucose values have been associated with increased cardiovascular risk independent of FPG in some epidemiological studies. In diabetic subjects, some surro- gate measures of vascular pathology, such as endothelial dysfunction, are negatively affected by postprandial hyperglycemia (60). It is clear that postprandial hyperglycemia, like preprandial hyperglycemia, contributes to elevated AlC levels, with its relative contribution being higher at AlC levels that are closer to 7\%. However, outcome studies have clearly shown AlC to be the primary predictor of complications, and landmark glycemic control trials such as the DCCT and UKPDS relied overwhelmingly on preprandial SMBG. Additionally, a randomized controlled trial presented at the 68th Scientific Sessions of the American Diabetes Association in June 2008 found no CVD benefit of insulin regimens targeting postprandial glucose compared with those targeting preprandial glucose. A reasonable recommendation for postprandial testing and targets is that for individuals who have premeal glucose values within target but have AlC values above target, monitoring postprandial plasma glucose (PPG) $1-2 \mathrm{~h}$ after the start of the meal and treatment aimed at reducing PPG values to $<180 \mathrm{mg} / \mathrm{dl}$ may help lower AlC.

As noted above, less stringent treatment goals may be appropriate for adults with limited life expectancies or advanced vascular disease. Glycemic goals for children are provided in Section VII.A.1.a. Severe or frequent hypoglycemia is an absolute indication for the modification of treatment regimens, including setting higher glycemic goals.

Regarding goals for glycemic control for women with GDM, recommendations from the Fifth International WorkshopConference on Gestational Diabetes Mellitus (61) were to target the following maternal capillary glucose concentrations:

- preprandial: $\leq 95 \mathrm{mg} / \mathrm{dl}(5.3 \mathrm{mmol} / \mathrm{l})$ and either

- 1-h postmeal: $\leq 140 \mathrm{mg} / \mathrm{dl}$ (7.8 mmol/) or

- 2-h postmeal: $\leq 120 \mathrm{mg} / \mathrm{dl}(6.7 \mathrm{mmol} / \mathrm{l})$

For women with preexisting type 1 or type 2 diabetes who become pregnant, a recent consensus statement (62) recommended the following as optimal glycemic goals, if they can be achieved without excessive hypoglycemia:

- premeal, bedtime, and overnight glucose 60-99 mg/dl 
- peak postprandial glucose 100-129 $\mathrm{mg} / \mathrm{dl}$

- $\mathrm{AlC}<6.0 \%$

\section{Approach to treatment}

a. Therapy for type 1 diabetes. The DCCT clearly showed that intensive insulin therapy (three or more injections per day of insulin or continuous subcutaneous insulin infusion (CSII, or insulin pump therapy) was a key part of improved glycemia and better outcomes (45). At the time of the study, therapy was carried out with short- and intermediateacting human insulins. Despite better microvascular outcomes, intensive insulin therapy was associated with a marked increase in severe hypoglycemia (62 episodes per 100 patient-years of therapy). Since the time of the DCCT, a number of rapid-acting and long-acting insulin analogs have been developed. These analogs were designed to be more "physiological" in their pharmacokinetics and pharmacodynamics and are associated with less hypoglycemia with equal AlC lowering in type 1 diabetes $(63,64)$.

Therefore, recommended therapy for type 1 diabetes consists of the following components: 1) use of multiple dose insulin injections (3-4 injections per day of basal and prandial insulin) or CSII therapy; 2) matching of prandial insulin to carbohydrate intake, premeal blood glucose, and anticipated activity; and 3) for many patients (especially if hypoglycemia is a problem), use of insulin analogs. There are excellent reviews available that guide the initiation and management of insulin therapy to achieve desired glycemic goals $(3,63,65)$.

b. Therapy for type 2 diabetes. The ADA and the European Association for the Study of Diabetes published a consensus statement on the approach to management of hyperglycemia in individuals with type 2 diabetes (66) and recently published an update (67). Highlights of this approach are: intervention at the time of diagnosis with metformin in combination with lifestyle changes (MNT and exercise) and continuing timely augmentation of therapy with additional agents (including early initiation of insulin therapy) as a means of achieving and maintaining recommended levels of glycemic control (i.e., AlC $<7 \%$ for most patients). The overall objective is to achieve and maintain glycemic control and to change interventions when therapeutic goals are not being met.
The algorithm took into account the evidence for AlC-lowering of the individual interventions, their additive effects, and their expense. The precise drugs used and their exact sequence may not be as important as achieving and maintaining glycemic targets safely. Medications not included in the consensus algorithm, owing to less glucose-lowering effectiveness, limited clinical data, and/or relative expense, still may be appropriate choices in individual patients to achieve glycemic goals. Initiation of insulin at time of diagnosis is recommended for individuals presenting with weight loss or other severe hyperglycemic symptoms or signs. For a list of currently approved diabetes medications, see http://ndep.nih.gov/diabetes/ pubs/Drug_tables_supplement.pdf.

\section{MNT}

\section{General recommendations}

- Individuals who have pre-diabetes or diabetes should receive individualized MNT as needed to achieve treatment goals, preferably provided by a registered dietitian familiar with the components of diabetes MNT. (B)

- MNT should be covered by insurance and other payors. (E)

\section{Energy balance, overweight, and obesity}

- In overweight and obese insulinresistant individuals, modest weight loss has been shown to reduce insulin resistance. Thus, weight loss is recommended for all overweight or obese individuals who have or are at risk for diabetes. (A)

- For weight loss, either low-carbohydrate or low-fat calorie restricted diets may be effective in the short-term (up to 1 year). (A)

- For patients on low-carbohydrate diets, monitor lipid profiles, renal function, and protein intake (in those with nephropathy) and adjust hypoglycemic therapy as needed. (E)

- Physical activity and behavior modification are important components of weight loss programs and are most helpful in maintenance of weight loss. (B)

\section{Primary prevention of diabetes}

- Among individuals at high risk for developing type 2 diabetes, structured programs that emphasize lifestyle changes that include moderate weight loss (7\% body weight) and regular physical activity (150 min/week), with dietary strategies including reduced calories and reduced intake of dietary fat, can reduce the risk for developing diabetes and are therefore recommended. (A)

- Individuals at high risk for type 2 diabetes should be encouraged to achieve the U.S. Department of Agriculture recommendation for dietary fiber (14 g fiber/1,000 $\mathrm{kcal}$ ) and foods containing whole grains (one-half of grain intake). (B)

\section{Dietary fat intake in diabetes management}

- Saturated fat intake should be $<7 \%$ of total calories. (A)

- Intake of trans fat should be minimized. (B)

\section{Carbohydrate intake in diabetes management}

- Monitoring carbohydrate, whether by carbohydrate counting, exchanges, or experience-based estimation, remains a key strategy in achieving glycemic control. (A)

- For individuals with diabetes, the use of the glycemic index and glycemic load may provide a modest additional benefit for glycemic control over that observed when total carbohydrate is considered alone. (B)

\section{Other nutrition recommendations}

- Sugar alcohols and nonnutritive sweeteners are safe when consumed within the acceptable daily intake levels established by the Food and Drug Administration (FDA). (A)

- If adults with diabetes choose to use alcohol, daily intake should be limited to a moderate amount (one drink per day or less for adult women and two drinks per day or less for adult men). (E)

- Routine supplementation with antioxidants, such as vitamins $E$ and $C$ and carotene, is not advised because of lack of evidence of efficacy and concern related to long-term safety. (A)

- Benefit from chromium supplementation in people with diabetes or obesity has not been conclusively demonstrated and, therefore, cannot be recommended. (E)

MNT is an integral component of diabetes prevention, management, and selfmanagement education. In addition to its role in preventing and controlling diabetes, ADA recognizes the importance of nutrition as an essential component of an overall healthy lifestyle. A full review of the evidence regarding nutrition in pre- 
venting and controlling diabetes and its complications and additional nutritionrelated recommendations can be found in the ADA position statement "Nutrition Recommendations and Interventions for Diabetes," published in 2007 and updated for 2008 (68). Achieving nutritionrelated goals requires a coordinated team effort that includes the active involvement of the person with pre-diabetes or diabetes. Because of the complexity of nutrition issues, it is recommended that a registered dietitian who is knowledgeable and skilled in implementing nutrition therapy into diabetes management and education be the team member who provides MNT.

Clinical trials/outcome studies of MNT have reported decreases in AlC at 3-6 months ranging from 0.25 to $2.9 \%$ with higher reductions seen in type 2 diabetes of shorter duration. Multiple studies have demonstrated sustained improvements in AlC at 12 months and longer when a registered dietician provided follow-up visits ranging from monthly to three sessions per year (6976). Meta-analyses of studies in nondiabetic, free-living subjects report that MNT reduces LDL cholesterol by $15-25 \mathrm{mg} / \mathrm{dl}$ (77) or can lower LDL cholesterol by up to $16 \%$ (78), while clinical trials support a role for lifestyle modification in treating hypertension $(78,79)$.

Because of the effects of obesity on insulin resistance, weight loss is an important therapeutic objective for overweight or obese individuals with prediabetes or diabetes (80). Short-term studies have demonstrated that moderate weight loss ( $5 \%$ of body weight) in subjects with type 2 diabetes is associated with decreased insulin resistance, improved measures of glycemia and lipemia, and reduced blood pressure (81); longerterm studies (52 weeks) showed mixed effects on A1C in adults with type 2 diabetes (82-85), and results were confounded by pharmacologic weight loss therapy. A systematic review of 80 weight loss studies of $\geq 1$ year duration demonstrated that moderate weight loss achieved through diet alone, diet and exercise, and meal replacements can be achieved and maintained over the long term (4.8-8\% weight loss at 12 months) (86). The multifactorial intensive lifestyle intervention employed in the DPP, which included reduced intake of fat and calories, led to weight loss averaging $7 \%$ at 6 months and maintenance of 5\% weight loss at 3 years, associated with a 58\% reduction in incidence of type 2 diabetes
(10). Look AHEAD (Action for Health in Diabetes) is a large clinical trial designed to determine whether long-term weight loss will improve glycemia and prevent cardiovascular events in subjects with type 2 diabetes. One-year results of the intensive lifestyle intervention in this trial show an average of $8.6 \%$ weight loss, significant reduction of $\mathrm{AlC}$, and reduction in several CVD risk factors (87). When completed, the Look AHEAD trial should provide insight into the effects of longterm weight loss on important clinical outcomes.

The optimal macronutrient distribution of weight loss diets has not been established. Although low-fat diets have traditionally been promoted for weight loss, several randomized controlled trials found that subjects on low-carbohydrate diets ( $<130 \mathrm{~g} /$ day of carbohydrate) lost more weight at 6 months than subjects on low-fat diets $(88,89)$; however, at 1 year, the difference in weight loss between the low-carbohydrate and low-fat diets was not significant, and weight loss was modest with both diets. Another study of overweight women randomized to one of four diets showed significantly more weight loss at 12 months with the Atkins lowcarbohydrate diet than with highercarbohydrate diets (90). Changes in serum triglyceride and HDL cholesterol were more favorable with the lowcarbohydrate diets. In one study, those subjects with type 2 diabetes demonstrated a greater decrease in AlC with a low-carbohydrate diet than with a low-fat diet (89). A recent meta-analysis showed that at 6 months, low-carbohydrate diets were associated with greater improvements in triglyceride and HDL cholesterol concentrations than low-fat diets; however, LDL cholesterol was significantly higher on the low-carbohydrate diets (91). In a 2-year dietary intervention study, Mediterranean and low-carbohydrate diets were found to be effective and safe alternatives to a low-fat diet for weight reduction in moderately obese participants (85).

The recommended dietary allowance for digestible carbohydrate is $130 \mathrm{~g} /$ day and is based on providing adequate glucose as the required fuel for the central nervous system without reliance on glucose production from ingested protein or fat. Although brain fuel needs can be met on lower carbohydrate diets, long-term metabolic effects of very-low-carbohydrate diets are unclear, and such diets eliminate many foods that are important sources of energy, fiber, vitamins, and minerals that are important in dietary palatability (92).

Although numerous studies have attempted to identify the optimal mix of macronutrients for meal plans of people with diabetes, it is unlikely that one such combination of macronutrients exists. The best mix of carbohydrate, protein, and fat appears to vary depending on individual circumstances. For those individuals seeking guidance as to macronutrient distribution in healthy adults, the Dietary Reference Intake (DRI) system may be helpful (92). It must be clearly recognized that regardless of the macronutrient mix, total caloric intake must be appropriate for the weight management goal. Further, individualization of the macronutrient composition will depend on the metabolic status of the patient (e.g., lipid profile, renal function) and/or food preferences. Individuals who choose to consume plant-based diets that are well planned and nutritionally adequate (i.e., vegetarian) may continue, as this can be done without being deleterious to metabolic control $(93,94)$.

The primary goal with respect to dietary fat in individuals with diabetes is to limit saturated fatty acids, trans fatty acids, and cholesterol intake so as to reduce risk for CVD. Saturated and trans fatty acids are the principal dietary determinants of plasma LDL cholesterol. There is a lack of evidence on the effects of specific fatty acids on people with diabetes, so the recommended goals are consistent with those for individuals with CVD $(78,95)$.

The FDA has approved five nonnutritive sweeteners for use in the U.S.: acesulfame potassium, aspartame, neotame, saccharin, and sucralose. Before being allowed on the market, all underwent rigorous scrutiny and were shown to be safe when consumed by the public, including people with diabetes and women during pregnancy. Reduced calorie sweeteners approved by the FDA include sugar alcohols (polyols) such as erythritol, isomalt, lactitol, maltitol, mannitol, sorbitol, xylitol, tagatose, and hydrogenated starch hydrolysates. The use of sugar alcohols appears to be safe; however, they may cause diarrhea, especially in children.

\section{Reimbursement for MNT}

MNT, when delivered by a registered dietitian according to nutrition practice guidelines, is reimbursed as part of the Medicare program as overseen by the Centers for Medicare and Medicaid Ser- 
vices (CMS) (www.cms.hhs.gov/ medicalnutritiontherapy)

\section{E. Bariatric surgery}

\section{Recommendations}

- Bariatric surgery should be considered for adults with BMI $\geq 35 \mathrm{~kg} / \mathrm{m}^{2}$ and type 2 diabetes, especially if the diabetes is difficult to control with lifestyle and pharmacologic therapy. (B)

- Patients with type 2 diabetes who have undergone bariatric surgery need lifelong lifestyle support and medical monitoring. (E)

- Although small trials have shown glycemic benefit of bariatric surgery in patients with type 2 diabetes and BMI of 30-35 $\mathrm{kg} / \mathrm{m}^{2}$, there is currently insufficient evidence to generally recommend surgery in patients with BMI $<35 \mathrm{~kg} / \mathrm{m}^{2}$ outside of a research protocol. (E)

- The long-term benefits, costeffectiveness, and risks of bariatric surgery in individuals with type 2 diabetes should be studied in well-designed randomized controlled trials with optimal medical and lifestyle therapy as the comparator. (E)

Gastric reduction surgery, either gastric banding or procedures that involve bypassing or transposing sections of the small intestine, when part of a comprehensive team approach, can be an effective weight loss treatment for severe obesity, and national guidelines support its consideration for people with type 2 diabetes who have BMI at or exceeding 35 $\mathrm{kg} / \mathrm{m}^{2}$. Bariatric surgery has been shown to lead to near or complete normalization of glycemia in 55-95\% of patients with type 2 diabetes, depending on the surgical procedure. A meta-analysis of studies of bariatric surgery reported that $78 \%$ of individuals with type 2 diabetes had complete "resolution" of diabetes (normalization of blood glucose levels in the absence of medications), and that the resolution rates were sustained in studies that had follow-up exceeding 2 years (96). Resolution rates are lowest with procedures that only constrict the stomach and higher with those that bypass portions of the small intestine. Additionally, there is increasing evidence that intestinal bypass procedures may have glycemic effects that are independent of, and additive to, their effects on weight.

A recent randomized controlled trial compared adjustable gastric banding to "best available" medical and lifestyle therapy in subjects with type 2 diabetes diagnosed less than 2 years before randomization and BMI $30-40 \mathrm{~kg} / \mathrm{m}^{2}$ (97). In this trial, $73 \%$ of surgically treated patients achieved "remission" of their diabetes, compared with $13 \%$ of those treated medically. The latter group lost only $1.7 \%$ of body weight, suggesting that their therapy was not optimal. Overall, the trial had 60 subjects, and only 13 had a BMI under $35 \mathrm{~kg} / \mathrm{m}^{2}$, making it difficult to generalize these results widely to diabetic patients who are less severely obese or with longer duration of diabetes.

Bariatric surgery is costly in the short term and has some risks. Rates of morbidity and mortality directly related to the surgery have been reduced considerably in recent years, with 30-day mortality rates now $0.28 \%$, similar to those of laparoscopic cholecystectomy (98). Longerterm concerns include vitamin and mineral deficiencies, osteoporosis, and rare but often severe hypoglycemia from insulin hypersecretion. Cohort studies attempting to match subjects suggest that the procedure may reduce longer-term mortality rates (99), and it is reasonable to postulate that there may be recouping of costs over the long run. However, studies of the mechanisms of glycemic improvement, long-term benefits and risks, and cost-effectiveness of bariatric surgery in individuals with type 2 diabetes will require well-designed randomized clinical trials, with optimal medical and lifestyle therapy of diabetes and cardiovascular risk factors as the comparitor.

\section{F. DSME}

\section{Recommendations}

- People with diabetes should receive DSME according to national standards when their diabetes is diagnosed and as needed thereafter. (B)

- Self-management behavior change is the key outcome of DSME and should be measured and monitored as part of care. (E)

- DSME should address psychosocial issues, since emotional well-being is strongly associated with positive diabetes outcomes. (C)

- DSME should be reimbursed by thirdparty payors. (E)

DSME is an essential element of diabetes care (100-106), and National Standards for DSME (107) are based on evidence for its benefits. Education helps people with diabetes initiate effective self-care when they are first diagnosed. Ongoing DSME also helps people with diabetes maintain effective self-management as their diabetes presents new challenges and treatment advances become available. DSME helps patients optimize metabolic control, prevent and manage complications, and maximize quality of life in a cost-effective manner (108).

Since the 1990s, there has been a shift from a didactic approach, with DSME focusing on providing information, to a skill-based approach that focuses on helping those with diabetes make informed self-management choices. Care of diabetes has shifted to an approach that is more patient centered and that places the person with diabetes, and joint decisionmaking with heath care professionals, at the center of the care model. Patientcentered care is respectful of and responsive to individual patient preferences, needs, and values and ensures that patient values guide all decision making (109).

\section{Evidence for the benefits of DSME}

Several studies have found that DSME is associated with improved diabetes knowledge and improved self-care behavior (101), improved clinical outcomes such as lower AlC $(102,103,105,106$, 110), lower self-reported weight (101), and improved quality of life (104). Better outcomes were reported for DSME interventions that were longer and included follow-up support (101), that were tailored to individual needs and preferences (100), and that addressed psychosocial issues $(100,101,105)$. Both individual and group approaches have been found effective $((111,112)$. There is increasing evidence for the role of a community health worker in delivering diabetes education in addition to the core team (113).

\section{National standards for DSME}

ADA-recognized DSME programs have staff that must be certified diabetes educators or have recent experience in diabetes education and management. The curriculum of ADA-recognized DSME programs must cover all nine areas of diabetes management, with the assessed needs of the individual determining which areas are addressed. The ADA Education Recognition Program (ERP) is a mechanism to ensure diabetes education programs meet the national standards and provide quality diabetes care. 


\section{Reimbursement for DSME}

DSME, when provided by a program that meets ADA ERP standards, is reimbursed as part of the Medicare program as overseen by the Centers for Medicare and Medicaid Services (CMS) (www.cms.hhs. gov/DiabetesSelfManagement).

\section{G. Physical activity}

\section{Recommendations}

- People with diabetes should be advised to perform at least $150 \mathrm{~min} /$ week of moderate-intensity aerobic physical activity $(50-70 \%$ of maximum heart rate). (A)

- In the absence of contraindications, people with type 2 diabetes should be encouraged to perform resistance training three times per week. (A)

ADA technical reviews on exercise in patients with diabetes have summarized the value of exercise in the diabetes management plan $(114,115)$. Regular exercise has been shown to improve blood glucose control, reduce cardiovascular risk factors, contribute to weight loss, and improve well being. Furthermore, regular exercise may prevent type 2 diabetes in high-risk individuals $(10-12)$. Structured exercise interventions of at least 8 weeks' duration have been shown to lower A1C by an average of $0.66 \%$ in people with type 2 diabetes, even with no significant change in BMI (116). Higher levels of exercise intensity are associated with greater improvements in AlC and in fitness (117).

\section{Frequency and type of exercise}

The U.S. Surgeon General's report (118) recommended that most adults accumulate at least 30 min of moderate-intensity activity on most, ideally all, days of the week. The studies included in the metaanalysis of effects of exercise interventions on glycemic control (116) had a mean number of sessions per week of 3.4, with a mean of 49 min per session. The DPP lifestyle intervention, which included 150 min per week of moderate intensity exercise, had a beneficial effect on glycemia in those with pre-diabetes. Therefore, it seems reasonable to recommend $\sim 150$ min of exercise per week for people with diabetes.

Resistance exercise improves insulin sensitivity to about the same extent as aerobic exercise (119). Clinical trials have provided strong evidence for the AlClowering value of resistance training in older adults with type 2 diabetes $(120,121)$ and for an additive benefit of combined aerobic and resistance exercise in adults with type 2 diabetes (122).

\section{Evaluation of the diabetic patient before recommending an exercise program}

Prior guidelines suggested that before recommending a program of physical activity, the provider should assess patients with multiple cardiovascular risk factors for coronary artery disease (CAD). As discussed more fully in Section VI.A.5, the area of screening asymptomatic diabetic patients for CAD remains unclear, and a recent $\mathrm{ADA}$ consensus statement on this issue concluded that routine screening is not recommended (123). Providers should use clinical judgment in this area. Certainly, high-risk patients should be encouraged to start with short periods of low-intensity exercise and increase the intensity and duration slowly.

Providers should assess patients for conditions that might contraindicate certain types of exercise or predispose to injury, such as uncontrolled hypertension, severe autonomic neuropathy, severe peripheral neuropathy or history of foot lesions, and advanced retinopathy. The patient's age and previous physical activity level should be considered.

\section{Exercise in the presence of nonoptimal glycemic control} Hyperglycemia. When people with type 1 diabetes are deprived of insulin for 12-48 $\mathrm{h}$ and are ketotic, exercise can worsen hyperglycemia and ketosis (124); therefore, vigorous activity should be avoided in the presence of ketosis. However, it is not necessary to postpone exercise based simply on hyperglycemia, provided the patient feels well and urine and/or blood ketones are negative.

Hypoglycemia. In individuals taking insulin and/or insulin secretagogues, physical activity can cause hypoglycemia if medication dose or carbohydrate consumption is not altered. For individuals on these therapies, added carbohydrate should be ingested if pre-exercise glucose levels are $<100 \mathrm{mg} / \mathrm{dl}(5.6 \mathrm{mmol} / \mathrm{l})$ $(125,126)$. Hypoglycemia is rare in diabetic individuals who are not treated with insulin or insulin secretagogues, and no preventive measures for hypoglycemia are usually advised in these cases.
Exercise in the presence of specific long-term complications of diabetes Retinopathy. In the presence of proliferative diabetic retinopathy (PDR) or severe nonproliferative diabetic retinopathy (NPDR), vigorous aerobic or resistance exercise may be contraindicated because of the risk of triggering vitreous hemorrhage or retinal detachment (127).

Peripheral neuropathy. Decreased pain sensation in the extremities results in increased risk of skin breakdown and infection and of Charcot joint destruction. Therefore, in the presence of severe peripheral neuropathy, it may be best to encourage non-weight-bearing activities such as swimming, bicycling, or arm exercises $(128,129)$.

Autonomic neuropathy. Autonomic neuropathy can increase the risk of exercise-induced injury or adverse events through decreased cardiac responsiveness to exercise, postural hypotension, impaired thermoregulation, impaired night vision due to impaired papillary reaction, and unpredictable carbohydrate delivery from gastroparesis predisposing to hypoglycemia (128). Autonomic neuropathy is also strongly associated with CVD in people with diabetes $(130,131)$. People with diabetic autonomic neuropathy should undergo cardiac investigation before beginning physical activity more intense than that to which they are accustomed.

Albuminuria and nephropathy. Physical activity can acutely increase urinary protein excretion. However, there is no evidence that vigorous exercise increases the rate of progression of diabetic kidney disease and likely no need for any specific exercise restrictions for people with diabetic kidney disease (132).

\section{H. Psychosocial assessment and care}

\section{Recommendations}

- Assessment of psychological and social situation should be included as an ongoing part of the medical management of diabetes. (E)

- Psychosocial screening and follow-up should include, but is not limited to, attitudes about the illness, expectations for medical management and outcomes, affect/mood, general and diabetes-related quality of life, resources (financial, social, and emotional), and psychiatric history. (E)

- Screen for psychosocial problems such as depression, anxiety, eating disorders, and cognitive impairment when 
adherence to the medical regimen is poor. (E)

Psychological and social problems can impair the individual's (133-138) or family's (139) ability to carry out diabetes care tasks and therefore compromise health status. There are opportunities for the clinician to assess psychosocial status in a timely and efficient manner so that referral for appropriate services can be accomplished.

Key opportunities for screening of psychosocial status occur at diagnosis, during regularly scheduled management visits, during hospitalizations, at discovery of complications, or when problems with glucose control, quality of life, or adherence are identified (140). Patients are likely to exhibit psychological vulnerability at diagnosis and when their medical status changes, i.e., the end of the honeymoon period, when the need for intensified treatment is evident, and when complications are discovered $(135,137)$.

Issues known to impact selfmanagement and health outcomes include but are not limited to attitudes about the illness, expectations for medical management and outcomes, affect/mood, general and diabetes-related quality of life, resources (financial, social, and emotional) (136), and psychiatric history $(137,140,141)$. Screening tools are available for a number of these areas (142). Indications for referral to a mental health specialist familiar with diabetes management may include gross noncompliance with medical regimen (by self or others) (141), depression with the possibility of self-harm $(134,143)$, debilitating anxiety (alone or with depression), indications of an eating disorder (144), or cognitive functioning that significantly impairs judgment (143). It is preferable to incorporate psychological assessment and treatment into routine care rather than waiting for identification of a specific problem or deterioration in psychological status (142). Although the clinician may not feel qualified to treat psychological problems, utilizing the patient-provider relationship as a foundation for further treatment can increase the likelihood that the patient will accept referral for other services. It is important to establish that emotional well-being is part of diabetes management (140).

\section{When treatment goals are not met}

For a variety of reasons, some people with diabetes and their health care providers do not achieve the desired goals of treat- ment (Table 9). Re-thinking the treatment regimen may require assessment of barriers to adherence including income, educational attainment, and competing demands, including those related to family responsibilities and family dynamics. Other strategies may include culturally appropriate and enhanced DSME, comanagement with a diabetes team, referral to a medical social worker for assistance with insurance coverage or change in pharmacological therapy. Initiation of or increase in SMBG, utilization of continuous glucose monitoring, frequent contact with the patient, or referral to an endocrinologist may be useful.

\section{J. Intercurrent illness}

The stress of illness, trauma, and/or surgery frequently aggravates glycemic control and may precipitate diabetic ketoacidosis (DKA) or nonketotic hyperosmolar state, life-threatening conditions that require immediate medical care to prevent complications and death (145). Any condition leading to deterioration in glycemic control necessitates more frequent monitoring of blood glucose and (in ketosis-prone patients) urine or blood ketones. Marked hyperglycemia requires temporary adjustment of the treatment program and, if accompanied by ketosis, vomiting, or alteration in level of consciousness, immediate interaction with the diabetes care team. The patient treated with noninsulin therapies or MNT alone may temporarily require insulin. Adequate fluid and caloric intake must be assured. Infection or dehydration are more likely to necessitate hospitalization of the person with diabetes than the person without diabetes.

The hospitalized patient should be treated by a physician with expertise in the management of diabetes. For further information on management of patients with hyperglycemia in the hospital, see Section VIII.A. For further information on management of DKA or nonketotic hyperosmolar state, refer to the ADA position statement on hyperglycemic crises (145)

\section{K. Hypoglycemia}

\section{Recommendations}

- Glucose (15-20 g) is the preferred treatment for the conscious individual with hypoglycemia, although any form of carbohydrate that contains glucose may be used. If SMBG 15 min after treatment shows continued hypoglycemia, the treatment should be repeated.
Once SMBG glucose returns to normal, the individual should consume a meal or snack to prevent recurrence of hypoglycemia. (E)

- Glucagon should be prescribed for all individuals at significant risk of severe hypoglycemia, and caregivers or family members of these individuals should be instructed in its administration. Glucagon administration is not limited to health care professionals. (E)

- Individuals with hypoglycemia unawareness or one or more episodes of severe hypoglycemia should be advised to raise their glycemic targets to strictly avoid further hypoglycemia for at least several weeks to partially reverse hypoglycemia unawareness and reduce risk of future episodes. (B)

Hypoglycemia is the leading limiting factor in the glycemic management of type 1 and insulin-treated type 2 diabetes (146). Treatment of hypoglycemia (plasma glucose $<70 \mathrm{mg} / \mathrm{dl}$ ) requires ingestion of glucose- or carbohydrate-containing foods. The acute glycemic response correlates better with the glucose content than with the carbohydrate content of the food. Although pure glucose is the preferred treatment, any form of carbohydrate that contains glucose will raise blood glucose. Added fat may retard and then prolong the acute glycemic response (147). Ongoing activity of insulin or insulin secretagogues may lead to recurrence of hypoglycemia unless further food is ingested after recovery.

Severe hypoglycemia (where the individual requires the assistance of another person and cannot be treated with oral carbohydrate due to confusion or unconsciousness) should be treated using emergency glucagon kits, which require a prescription. Those in close contact with, or having custodial care of, people with hypoglycemia-prone diabetes (family members, roommates, school personnel, child care providers, correctional institution staff, or coworkers) should be instructed in use of such kits. An individual does not need to be a health care professional to safely administer glucagon. Care should be taken to ensure that unexpired glucagon kits are available.

Prevention of hypoglycemia is a critical component of diabetes management. Teaching people with diabetes to balance insulin use, carbohydrate intake, and exercise is a necessary but not always sufficient strategy. In type 1 diabetes and severely insulin-deficient type 2 diabetes, 
the syndrome of hypoglycemia unawareness, or hypoglycemia-associated autonomic failure, can severely compromise stringent diabetes control and quality of life. The deficient counter-regulatory hormone release and autonomic responses in this syndrome are both risk factors for, and caused by, hypoglycemia. A corollary to this "vicious cycle" is that several weeks of avoidance of hypoglycemia has been demonstrated to improve counterregulation and awareness to some extent in many patients $(146,148,149)$. Hence, patients with one or more episodes of severe hypoglycemia may benefit from at least short-term relaxation of glycemic targets.

\section{Immunization}

\section{Recommendations}

- Annually provide an influenza vaccine to all diabetic patients $\geq 6$ months of age. (C)

- Administer pneumococcal polysaccharide vaccine to all diabetic patients $\geq 2$ years of age. A one-time revaccination is recommended for individuals $>64$ years of age previously immunized when they were $<65$ years of age if the vaccine was administered $>5$ years ago. Other indications for repeat vaccination include nephrotic syndrome, chronic renal disease, and other immunocompromised states, such as after transplantation. (C)

Influenza and pneumonia are common, preventable infectious diseases associated with high mortality and morbidity in the elderly and in people with chronic diseases. Though there are limited studies reporting the morbidity and mortality of influenza and pneumococcal pneumonia specifically in people with diabetes, observational studies of patients with a variety of chronic illnesses, including diabetes, show that these conditions are associated with an increase in hospitalizations for influenza and its complications. People with diabetes may be at increased risk of the bacteremic form of pneumococcal infection and have been reported to have a high risk of nosocomial bacteremia, which has a mortality rate as high as $50 \%$ (150).

Safe and effective vaccines are available that can greatly reduce the risk of serious complications from these diseases $(151,152)$. In a case-control series, influenza vaccine was shown to reduce diabetes-related hospital admission by as much as 79\% during flu epidemics (151). There is sufficient evidence to support that people with diabetes have appropriate serologic and clinical responses to these vaccinations. The Centers for Disease Control and Prevention's Advisory Committee on Immunization Practices recommends influenza and pneumococcal vaccines for all individuals with diabetes (http://www.cdc.gov/vaccines/recs/). For a complete discussion on the prevention of influenza and pneumococcal disease in people with diabetes, consult the technical review and position statement on this subject $(150,153)$.

\section{PREVENTION AND MANACEMENT OF diabetes complications}

\section{A. CVD}

CVD is the major cause of morbidity and mortality for individuals with diabetes and the largest contributor to the direct and indirect costs of diabetes. The common conditions coexisting with type 2 diabetes (e.g., hypertension and dyslipidemia) are clear risk factors for CVD, and diabetes itself confers independent risk. Numerous studies have shown the efficacy of controlling individual cardiovascular risk factors in preventing or slowing CVD in people with diabetes. Large benefits are seen when multiple risk factors are addressed globally (154). Evidence is summarized in the following sections and reviewed in detail in the ADA technical reviews on hypertension (155), dyslipidemia (156), aspirin therapy (157), and smoking cessation (158) and in the American Heart Association (AHA)/ADA scientific statement on prevention of CVD in people with diabetes (159).

\section{Hypertension/blood pressure control}

\section{Recommendations}

\section{Screening and diagnosis}

- Blood pressure should be measured at every routine diabetes visit. Patients found to have a systolic blood pressure of $\geq 130 \mathrm{mmHg}$ or a diastolic blood pressure of $\geq 80 \mathrm{mmHg}$ should have blood pressure confirmed on a separate day. Repeat systolic blood pressure of $\geq 130 \mathrm{mmHg}$ or diastolic blood pressure of $\geq 80 \mathrm{mmHg}$ confirms a diagnosis of hypertension. (C)
Goals

- Patients with diabetes should be treated to a systolic blood pressure $<130$ mmHg. (C)

- Patients with diabetes should be treated to a diastolic blood pressure $<80$ mmHg. (B)

\section{Treatment}

- Patients with a systolic blood pressure of 130-139 mmHg or a diastolic blood pressure of 80-89 mmHg may be given lifestyle therapy alone for a maximum of 3 months and then, if targets are not achieved, be treated with the addition of pharmacological agents. (E)

- Patients with more severe hypertension (systolic blood pressure $\geq 140$ mmHg or diastolic blood pressure $\geq 90 \mathrm{mmHg}$ ) at diagnosis or follow-up should receive pharmacologic therapy in addition to lifestyle therapy. (A)

- Pharmacologic therapy for patients with diabetes and hypertension should be with a regimen that includes either an ACE inhibitor or an angiotensin receptor blocker (ARB). If one class is not tolerated, the other should be substituted. If needed to achieve blood pressure targets, a thiazide diuretic should be added to those with an estimated GFR (see below) $\geq 30 \mathrm{ml} / \mathrm{min}$ per 1.73 $\mathrm{m}^{2}$ and a loop diuretic for those with an estimated GFR $<30 \mathrm{ml} / \mathrm{min}$ per 1.73 $\mathrm{m}^{2}$. (C)

- Multiple drug therapy (two or more agents at maximal doses) is generally required to achieve blood pressure targets. (B)

- If ACE inhibitors, ARBs, or diuretics are used, kidney function and serum potassium levels should be closely monitored. (E)

- In pregnant patients with diabetes and chronic hypertension, blood pressure target goals of 110-129/65-79 mmHg are suggested in the interest of longterm maternal health and minimizing impaired fetal growth. ACE inhibitors and ARBs are contraindicated during pregnancy. (E)

Hypertension is a common comorbidity of diabetes, affecting the majority of patients, with prevalence depending on type of diabetes, age, obesity, and ethnicity. Hypertension is a major risk factor for both CVD and microvascular complications. In type 1 diabetes, hypertension is often the result of underlying nephropa- 
thy, while in type 2 it usually coexists with other cardiometabolic risk factors.

\section{Screening and diagnosis}

Measurement of blood pressure in the office should be done by a trained individual and follow the guidelines established for nondiabetic individuals: measurement in the seated position, with feet on the floor and arm supported at heart level, after 5 min of rest. Cuff size should be appropriate for the upper arm circumference. Elevated values should be confirmed on a separate day. Because of the clear synergistic risks of hypertension and diabetes, the diagnostic cutoff for a diagnosis of hypertension is lower in people with diabetes (blood pressure $\geq 130 / 80$ ) than in those without diabetes (blood pressure $\geq 140 / 90 \mathrm{mmHg}$ ) (160).

Home blood pressure self-monitoring and 24-h ambulatory blood pressure monitoring may provide additional evidence of "white coat" and masked hypertension and other discrepancies between office and "true" blood pressure, and studies in nondiabetic populations show that home measurements may better correlate with CVD risk than office measurements $(161,162)$. However, the preponderance of the clear evidence of benefits of treatment of hypertension in people with diabetes is based on office measurements.

\section{Treatment goals}

Randomized clinical trials have demonstrated the benefit (reduction of CHD events, stroke, and nephropathy) of lowering blood pressure to $<140 \mathrm{mmHg}$ systolic and $<80 \mathrm{mmHg}$ diastolic in individuals with diabetes (160,163-165). Epidemiologic analyses show that blood pressure $>115 / 75 \mathrm{mmHg}$ is associated with increased cardiovascular event rates and mortality in individuals with diabetes $(160,166,167)$. Therefore, a target blood pressure goal of $<130 / 80 \mathrm{mmHg}$ is reasonable if it can be safely achieved. The ongoing ACCORD trial is designed to determine whether blood pressure lowering to systolic blood pressure $<120 \mathrm{mmHg}$ provides greater cardiovascular protection than a systolic blood pressure level of $<140 \mathrm{mmHg}$ in patients with type 2 diabetes (www.accord.org).

\section{Treatment strategies}

Although there are no well-controlled studies of diet and exercise in the treatment of hypertension in individuals with diabetes, studies in nondiabetic individu- als have shown anti-hypertensive effects similar to pharmacologic monotherapy of reducing sodium intake and excess body weight; increasing consumption of fruits, vegetables, and low-fat dairy products; avoiding excessive alcohol consumption; and increasing activity levels $(160,168)$. These nonpharmacological strategies may also positively affect glycemia and lipid control. Their effects on cardiovascular events have not been established. An initial trial of nonpharmacologic therapy may be reasonable in diabetic individuals with mild hypertension (systolic blood pressure 130-139 mmHg or diastolic blood pressure $80-89 \mathrm{mmHg}$ ). If the blood pressure is $\geq 140 \mathrm{mmHg}$ systolic and/or $\geq 90 \mathrm{mmHg}$ diastolic at the time of diagnosis, pharmacologic therapy should be initiated along with nonpharmacologic therapy (160).

Lowering of blood pressure with regimens based on a variety of antihypertensive drugs, including ACE inhibitors, ARBs, $\beta$-blockers, diuretics, and calcium channel blockers, has been shown to be effective in reducing cardiovascular events. Several studies have suggested that ACE inhibitors may be superior to dihydropyridine calcium channel blockers in reducing cardiovascular events (169-171). However, a variety of other studies have shown no specific advantage to ACE inhibitors as initial treatment of hypertension in the general hypertensive population, but rather an advantage on cardiovascular outcomes of initial therapy with low-dose thiazide diuretics (160, 172,173).

In people with diabetes, inhibitors of the renin-angiotensin system (RAS) may have unique advantages for initial or early therapy of hypertension. In a nonhypertension trial of high-risk individuals, including a large subset with diabetes, an ACE inhibitor reduced CVD outcomes (174). In patients with congestive heart failure (CHF), including diabetic subgroups, ARBs have been shown to reduce major CVD outcomes (175-178), and in type 2 patients with significant nephropathy, ARBs were superior to calcium channel blockers for reducing heart failure (179-181). Though evidence for distinct advantages of RAS inhibitors on CVD outcomes in diabetes remains conflicting $(163,182)$, the high CVD risks associated with diabetes, and the high prevalence of undiagnosed CVD, may still favor recommendations for their use as first-line hypertension therapy in people with diabetes (160). Recently, the blood pressure arm of the ADVANCE trial demonstrated that routine administration of a fixed combination of the ACE inhibitor perindopril and the diuretic indapamide significantly reduced combined microvascular and macrovascular outcomes, as well as CVD and total mortality. The improved outcomes could also have been due to lower achieved blood pressure in the perindopril-indapamide arm (183). The compelling benefits of RAS inhibitors in diabetic patients with albuminuria or renal insufficiency provide additional rationale for use of these agents (see section VI.B below).

An important caveat is that most patients with hypertension require multidrug therapy to reach treatment goals, especially diabetic patients whose targets are lower. Many patients will require three or more drugs to reach target goals (160). If blood pressure is refractory to multiple agents, clinicians should consider an evaluation for secondary forms of hypertension.

During pregnancy in diabetic women with chronic hypertension, target blood pressure goals of systolic blood pressure 110-129 mmHg and diastolic blood pressure 65-79 $\mathrm{mmHg}$ are reasonable, as they contribute to long-term maternal health. Lower blood pressure levels may be associated with impaired fetal growth. During pregnancy, treatment with ACE inhibitors and ARBs is contraindicated, since they are likely to cause fetal damage. Antihypertensive drugs known to be effective and safe in pregnancy include methyldopa, labetalol, diltiazem, clonidine, and prazosin. Chronic diuretic use during pregnancy has been associated with restricted maternal plasma volume, which might reduce uteroplacental perfusion (184).

\section{Dyslipidemia/lipid management}

\section{Recommendations}

\section{Screening}

- In most adult patients, measure fasting lipid profile at least annually. In adults with low-risk lipid values (LDL cholesterol $<100 \mathrm{mg} / \mathrm{dl}$, HDL cholesterol $>50 \mathrm{mg} / \mathrm{dl}$, and triglycerides <150 $\mathrm{mg} / \mathrm{dl}$ ), lipid assessments may be repeated every 2 years. (E)

Treatment recommendations and goals

- Lifestyle modification focusing on the reduction of saturated fat, trans fat, and cholesterol intake; weight loss (if indicated); and increased physical activity 
Table 10-Reduction in 10-year risk of major CVD end points (CHD death/non-fatal MI) in major statin trials, or substudies of major trials, in diabetic subjects $(\mathrm{n}=16,032)$

\begin{tabular}{|c|c|c|c|c|c|c|}
\hline Study (ref.) & $\begin{array}{c}\text { CVD } \\
\text { prevention } \\
\end{array}$ & Statin dose and comparator & $\begin{array}{l}\text { Risk reduction } \\
(\%)\end{array}$ & $\begin{array}{c}\text { Relative risk } \\
\text { reduction } \\
(\%)\end{array}$ & $\begin{array}{c}\text { Absolute risk } \\
\text { reduction } \\
(\%)\end{array}$ & $\begin{array}{c}\text { LDL cholesterol } \\
\text { reduction }\end{array}$ \\
\hline 4S-DM (186) & $2^{\circ}$ & Simvastatin $20-40 \mathrm{mg}$ vs. placebo & 85.7 to 43.2 & 50 & 42.5 & 186 to $119 \mathrm{mg} / \mathrm{dl}(36 \%)$ \\
\hline ASPEN $2^{\circ}(191)$ & $2^{\circ}$ & Atorvastatin $10 \mathrm{mg}$ vs. placebo & 39.5 to 24.5 & 34 & 12.7 & 112 to $79 \mathrm{mg} / \mathrm{dl}(29 \%)$ \\
\hline HPS-DM (187) & $2^{\circ}$ & Simvastatin $40 \mathrm{mg}$ vs. placebo & 43.8 to 36.3 & 17 & 7.5 & 123 to $84 \mathrm{mg} / \mathrm{dl}(31 \%)$ \\
\hline CARE-DM (188) & $2^{\circ}$ & Pravastatin 40 mg vs. placebo & 40.8 to 35.4 & 13 & 5.4 & 136 to $99 \mathrm{mg} / \mathrm{dl}(27 \%)$ \\
\hline TNT-DM (189) & $2^{\circ}$ & Atorvastatin $80 \mathrm{mg}$ vs. $10 \mathrm{mg}$ & 26.3 to 21.6 & 18 & 4.7 & 99 to 77 mg/dl (22\%) \\
\hline HPS-DM (187) & $1^{\circ}$ & Simvastatin 40 mg vs. placebo & 17.5 to 11.5 & 34 & 6.0 & 124 to 86 mg/dl (31\%) \\
\hline CARDS (209) & $1^{\circ}$ & Atorvastatin $10 \mathrm{mg}$ vs. placebo & 11.5 to 7.5 & 35 & 4 & 118 to $71 \mathrm{mg} / \mathrm{dl}(40 \%)$ \\
\hline ASPEN (191) & $1^{\circ}$ & Atorvastatin $10 \mathrm{mg}$ vs. placebo & 9.8 to 7.9 & 19 & 1.9 & 114 to $80 \mathrm{mg} / \mathrm{dl}(30 \%)$ \\
\hline ASCOT-DM (190) & $1^{\circ}$ & Atorvastatin $10 \mathrm{mg}$ vs. placebo & 11.1 to 10.2 & 8 & 0.9 & 125 to $82 \mathrm{mg} / \mathrm{dl}(34 \%)$ \\
\hline
\end{tabular}

Studies were of differing lengths (3.3-5.4 years) and used somewhat different outcomes, but all reported rates of CVD death and non-fatal MI. In this tabulation, results of the statin on 10-year risk of major CVD end points (CHD death/non-fatal MI) are listed for comparison between studies. Correlation between 10-year CVD risk of the control group and the absolute risk reduction with statin therapy is highly significant $(P=0.0007)$. Analyses provided by Craig Williams, Pharm.D., Oregon Health \& Science University, 2007.

should be recommended to improve the lipid profile in patients with diabetes. (A)

- Statin therapy should be added to lifestyle therapy, regardless of baseline lipid levels, for diabetic patients:

- with overt CVD (A)

- without CVD who are over the age of 40 and have one or more other CVD risk factors. (A)

- For lower-risk patients than the above (e.g., without overt CVD and under the age of 40), statin therapy should be considered in addition to lifestyle therapy if LDL cholesterol remains above $100 \mathrm{mg} / \mathrm{dl}$ or in those with multiple CVD risk factors. (E)

- In individuals without overt CVD, the primary goal is an LDL cholesterol $<100 \mathrm{mg} / \mathrm{dl}$ (2.6 mmol/l). (A)

- In individuals with overt CVD, a lower LDL cholesterol goal of $<70 \mathrm{mg} / \mathrm{dl}$ (1.8 $\mathrm{mmol} / \mathrm{l})$, using a high dose of a statin, is an option. (B)

- If drug-treated patients do not reach the above targets on maximal tolerated statin therapy, a reduction in LDL cholesterol of $\sim 30-40 \%$ from baseline is an alternative therapeutic goal. (A)

- Triglycerides levels $<150$ mg/dl $(1.7$ $\mathrm{mmol} / \mathrm{l}$ ) and HDL cholesterol $>40$ $\mathrm{mg} / \mathrm{dl}(1.0 \mathrm{mmol} / \mathrm{l})$ in men and $>50$ $\mathrm{mg} / \mathrm{dl}(1.3 \mathrm{mmol} / \mathrm{l})$ in women are desirable. However, LDL cholesteroltargeted statin therapy remains the preferred strategy. (C)

- If targets are not reached on maximally tolerated doses of statins, combination therapy using statins and other lipidlowering agents may be considered to achieve lipid targets but has not been evaluated in outcome studies for either CVD outcomes or safety. (E)

- Statin therapy is contraindicated in pregnancy. (E)

\section{Evidence for benefits of lipid- lowering therapy}

Patients with type 2 diabetes have an increased prevalence of lipid abnormalities, contributing to their high risk of CVD. For the past decade or more, multiple clinical trials demonstrated significant effects of pharmacologic (primarily statin) therapy on CVD outcomes in subjects with CHD and for primary CVD prevention (185). Subanalyses of diabetic subgroups of larger trials (186-190) and trials specifically in subjects with diabetes $(191,192)$ showed significant primary and secondary prevention of CVD

Table 11-Summary of recommendations for glycemic, blood pressure, and lipid control for adults with diabetes

\begin{tabular}{ll}
\hline AlC & $<7.0 \% *$ \\
Blood pressure & $<130 / 80 \mathrm{mmHg}$ \\
Lipids & $<100 \mathrm{mg} / \mathrm{dl}(<2.6 \mathrm{mmol} / \mathrm{l}) \dagger$ \\
$\quad$ LDL cholesterol & $<$
\end{tabular}

* Referenced to a nondiabetic range of 4.0-6.0\% using a DCCT-based assay. $†$ In individuals with overt CVD, a lower LDL cholesterol goal of $<70 \mathrm{mg} / \mathrm{dl}(1.8 \mathrm{mmol} / \mathrm{l})$, using a high dose of a statin, is an option.

events \pm CHD deaths in diabetic populations. As shown in Table 10, and similar to findings in nondiabetic subjects, reduction in "hard" CVD outcomes (CHD death and nonfatal MI) can be more clearly seen in diabetic subjects with high baseline CVD risk (known CVD and/or very high LDL cholesterol levels), but overall the benefits of statin therapy in people with diabetes at moderate or high risk for CVD are convincing.

Low levels of HDL cholesterol, often associated with elevated triglyceride levels, are the most prevalent pattern of dyslipidemia in persons with type 2 diabetes. However, the evidence base for drugs that target these lipid fractions is significantly less robust than that for statin therapy (193). Nicotinic acid has been shown to reduce CVD outcomes (194), although the study was done in a nondiabetic cohort. Gemfibrozil has been shown to decrease rates of CVD events in subjects without diabetes $(195,196)$ and in the diabetic subgroup in one of the larger trials (195). However, in a large trial specific to diabetic patients, fenofibrate failed to reduce overall cardiovascular outcomes (197).

\section{Dyslipidemia treatment and target lipid levels}

For most patients with diabetes, the first priority of dyslipidemia therapy (unless severe hypertriglyceridemia is the immediate issue) is to lower LDL cholesterol to a target goal of $<100 \mathrm{mg} / \mathrm{dl}(2.60 \mathrm{mmol} / \mathrm{l})$ (198). Lifestyle intervention, including MNT, increased physical activity, weight loss, and smoking cessation, may allow 
some patients to reach lipid goals. Nutrition intervention should be tailored according to each patient's age, type of diabetes, pharmacological treatment, lipid levels, and other medical conditions and should focus on the reduction of saturated fat, cholesterol, and trans unsaturated fat intake. Glycemic control can also beneficially modify plasma lipid levels, particularly in patients with very high triglycerides and poor glycemic control.

In those with clinical CVD or over age 40 with other CVD risk factors, pharmacological treatment should be added to lifestyle therapy regardless of baseline lipid levels. Statins are the drugs of choice for LDL cholesterol lowering.

In patients other than those described above, statin treatment should be considered if there is an inadequate LDL cholesterol response to lifestyle modifications and improved glucose control, or if the patient has increased cardiovascular risk (e.g., multiple cardiovascular risk factors or long duration of diabetes). Very little clinical trial evidence exists for type 2 patients under the age of 40 , or for type 1 patients of any age. In the Heart Protection Study, the subgroup of 600 patients with type 1 diabetes (lower age limit 40 years) had a proportionately similar reduction in risk as patients with type 2 diabetes, although not statistically significant (187). Although the data are not definitive, consideration should be given to similar lipid-lowering goals in type 1 diabetic patients as those in type 2 diabetic patients, particularly if they have other cardiovascular risk factors.

\footnotetext{
Alternative LDL cholesterol goals

Virtually all trials of statins and CVD outcomes have tested specific doses of statins against placebo, other doses of statin, or other statins, rather than aiming for specific LDL cholesterol goals (199). As can be seen in Table 10, placebo-controlled trials generally achieved LDL cholesterol reductions of $30-40 \%$ from baseline. Hence, LDL cholesterol lowering of this magnitude is an acceptable outcome for patients who cannot reach LDL cholesterol goals due to severe baseline elevations in LDL cholesterol and/or intolerance of maximal, or any, statin doses. Additionally, for those with baseline LDL cholesterol minimally above 100 $\mathrm{mg} / \mathrm{dl}$, prescribing statin therapy to lower LDL cholesterol about 30-40\% from baseline is probably more effective than prescribing just enough to get LDL cholesterol slightly below $100 \mathrm{mg} / \mathrm{dl}$.
}

Recent clinical trials in high-risk patients, such as those with acute coronary syndromes or previous cardiovascular events (200-202), have demonstrated that more aggressive therapy with high doses of statins to achieve an LDL cholesterol of $<70 \mathrm{mg} / \mathrm{dl}$ led to a significant reduction in further events. Therefore, a reduction in LDL cholesterol to a goal of $<70 \mathrm{mg} / \mathrm{dl}$ is an option in very-high-risk diabetic patients with overt CVD (203).

In individual patients, LDL cholesterol lowering with statins is highly variable, and this variable response is poorly understood (204). Reduction of CVD events with statins correlates very closely with LDL cholesterol lowering (185). When maximally tolerated doses of statins fail to significantly lower LDL cholesterol $(<30 \%$ reduction from patients baseline), the primary aim of combination therapy should be to achieve additional LDL cholesterol lowering. Niacin, fenofibrate, ezetimibe, and bile acid sequestrants all offer additional LDL cholesterol lowering. The evidence that combination therapy provides a significant increment in CVD risk reduction over statin therapy alone is still elusive.

\section{Treatment of other lipoprotein fractions or targets}

Severe hypertriglyceridemia may warrant immediate therapy of this abnormality with lifestyle and usually pharmacologic therapy (fibric acid derivative or niacin) to reduce the risk of acute pancreatitis. In the absence of severe hypertriglyceridemia, therapy targeting HDL cholesterol or triglycerides has intuitive appeal but lacks the evidence base of statin therapy (162). If the HDL cholesterol is $<40 \mathrm{mg} / \mathrm{dl}$ and the LDL cholesterol is between 100 and $129 \mathrm{mg} / \mathrm{dl}$, gemfibrozil or niacin might be used, especially if a patient is intolerant to statins. Niacin is the most effective drug for raising HDL cholesterol. It can significantly increase blood glucose at high doses, but recent studies demonstrate that at modest doses (750-2,000 mg/day), significant improvements in LDL cholesterol, HDL cholesterol, and triglyceride levels are accompanied by only modest changes in glucose that are generally amenable to adjustment of diabetes therapy (205,206).

Combination therapy, with a statin and a fibrate or a statin and niacin, may be efficacious for treatment for all three lipid fractions, but this combination is associated with an increased risk for abnormal transaminase levels, myositis, or rhabdo- myolysis. The risk of rhabdomyolysis is higher with higher doses of statins and with renal insufficiency and seems to be lower when statins are combined with fenofibrate than gemfibrozil (207). Several ongoing trials may provide much-needed evidence for the effects of combination therapy on cardiovascular outcomes.

In 2008, a consensus panel convened by ADA and the American College of Cardiology recommended a greater focus on non-HDL cholesterol and apolipoprotein B (apo B) in patients who are likely to have small LDL particles, such as people with diabetes (208). The consensus panel suggested that for statin-treated patients in whom the LDL cholesterol goal would be $<70 \mathrm{mg} /$ dl (non-HDL cholesterol $<100$ $\mathrm{mg} / \mathrm{dl}$ ), apo B should be measured and treated to $<80 \mathrm{mg} / \mathrm{dl}$. For patients on statins with an LDL cholesterol goal of $<100$ mg/dl (non-HDL cholesterol <130 $\mathrm{mg} / \mathrm{dl}$ ), apo B should be measured and treated to below $90 \mathrm{mg} / \mathrm{dl}$.

Table 11 summarizes the general recommendations for glycemic, blood pressure, and lipid control for adults with diabetes.

\section{Antiplatelet agents}

\section{Recommendations}

- Use aspirin therapy (75-162 mg/day) as a primary prevention strategy in those with type 1 or type 2 diabetes at increased cardiovascular risk, including those who are $>40$ years of age or who have additional risk factors (family history of CVD, hypertension, smoking, dyslipidemia, or albuminuria). (C)

- Use aspirin therapy (75-162 mg/day) as a secondary prevention strategy in those with diabetes with a history of CVD. (A)

- For patients with CVD and documented aspirin allergy, clopidogrel (75 mg/day) should be used. (B)

- Combination therapy with ASA (75$162 \mathrm{mg} /$ day) and clopidogrel (75 mg/ day) is reasonable for up to a year after an acute coronary syndrome. (B)

- Aspirin therapy is not recommended in people under 30 years of age due to lack of evidence of benefit and is contraindicated in patients under the age of 21 years because of the associated risk of Reye's syndrome. (E)

The use of aspirin in diabetes is reviewed in detail in the ADA technical review (157) and position statement (210) on this topic. Aspirin has been recom- 
mended for primary $(211,212)$ and secondary $(213,214)$ prevention of cardiovascular events in high-risk diabetic and nondiabetic individuals. One large meta-analysis and several clinical trials demonstrate the efficacy of using aspirin as a preventive measure for cardiovascular events, including stroke and myocardial infarction. Many trials have shown an $\sim 30 \%$ decrease in myocardial infarction and a 20\% decrease in stroke in a wide range of patients, including young and middle-aged patients, patients with and without a history of CVD, men and women, and patients with hypertension.

Dosages used in most clinical trials ranged from 75 to $325 \mathrm{mg} /$ day. There is little evidence to support any specific dose, but using the lowest possible dosage may help reduce side effects (215). Conversely, a randomized trial of $100 \mathrm{mg}$ of aspirin daily showed less of a primary prevention effect, without statistical significance, in the large diabetic subgroup in contrast to significant benefit in those without diabetes (216), raising the issue of aspirin resistance in those with diabetes.

The systematic review of evidence for the U.S. Preventive Services Task Force (USPSTF) estimated that aspirin reduced the risk for nonfatal and fatal MI (odds ratio 0.72 [95\% CI 0.60-0.87]). The review acknowledged the low numbers of diabetic subjects in most trials but concluded that subset analyses and a single trial in diabetic patients suggested that the estimates extended to those with diabetes (211). The USPSTF stated that the riskto-benefit ratio favors aspirin use when 5 -year CHD risk equals or exceeds 3\% and suggested aspirin therapy be considered for men $>40$ years of age, postmenopausal women, and younger persons with CHD risk factors (including diabetes) (212).

There is no evidence for a specific age at which to start aspirin, but aspirin has not been studied at ages $<30$ years.

Clopidogrel has been demonstrated to reduce CVD events in diabetic individuals (217). Adjunctive therapy in the first year after acute coronary syndrome in very-high-risk patients, or as alternative therapy in aspirin-intolerant patients, should be considered.

\section{Smoking cessation}

\section{Recommendations}

- Advise all patients not to smoke. (A)

- Include smoking cessation counseling and other forms of treatment as a routine component of diabetes care. (B)

Issues of smoking in diabetes are reviewed in detail in the ADA technical review (158) and position statement (218) on this topic. A large body of evidence from epidemiological, case-control, and cohort studies provides convincing documentation of the causal link between cigarette smoking and health risks. Cigarette smoking contributes to one of every five deaths in the U.S. and is the most important modifiable cause of premature death. Much of the prior work documenting the impact of smoking on health did not separately discuss results on subsets of individuals with diabetes, suggesting that the identified risks are at least equivalent to those found in the general population. Other studies of individuals with diabetes consistently found a heightened risk of CVD and premature death among smokers. Smoking is also related to the premature development of microvascular complications of diabetes and may have a role in the development of type 2 diabetes.

A number of large randomized clinical trials have demonstrated the efficacy and cost-effectiveness of smoking cessation counseling in changing smoking behavior and reducing tobacco use. The routine and thorough assessment of tobacco use is important as a means of preventing smoking or encouraging cessation. Special considerations should include assessment of level of nicotine dependence, which is associated with difficulty in quitting and relapse $(219,220)$. Free telephone quit lines are available in each state (see www.naquitline.org).

\section{CHD screening and treatment}

\section{Recommendations}

\section{Screening}

- In asymptomatic patients, evaluate risk factors to stratify patients by 10-year risk, and treat risk factors accordingly. (B)

\section{Treatment}

- In patients with known CVD, ACE inhibitor (C), aspirin (A), and statin therapy (A) (if not contraindicated) should be used to reduce the risk of cardiovascular events.

- In patients with a prior myocardial infarction, add $\beta$-blockers (if not contraindicated) to reduce mortality. (A)

- In patients $>40$ years of age with another cardiovascular risk factor (hypertension, family history, dyslipidemia, microalbuminuria, cardiac autonomic neuropathy, or smoking), aspirin and statin therapy (if not contraindicated) should be used to reduce the risk of cardiovascular events. (B)

- In patients with CHF, TZD use is contraindicated. (C)

- Metformin may be used in patients with stable CHF if renal function is normal. It should be avoided in unstable or hospitalized patients with CHF. (C)

Screening for CAD is reviewed in a recently updated consensus statement (123). To identify the presence of CAD in diabetic patients without clear or suggestive symptoms, a risk factor-based approach to the initial diagnostic evaluation and subsequent follow-up has intuitive appeal. However, recent studies concluded that using this approach fails to identify which patients will have silent ischemia on screening tests $(130,221)$.

Candidates for cardiac testing include those with 1) typical or atypical cardiac symptoms and 2) an abnormal resting electrocardiogram (ECG). The screening of asymptomatic patients remains controversial, especially as intensive medical therapy indicated in diabetic patients at high risk for CVD has an increasing evidence base for providing equal outcomes to invasive revascularization, including in diabetic patients (222). There is also recent preliminary evidence that silent myocardial ischemia may reverse over time, adding to the controversy concerning aggressive screening strategies (223). Finally, a recent randomized observational trial presented at the ADA's Scientific Sessions in June 2008 demonstrated no clinical benefit to routine screening of asymptomatic patients with type 2 diabetes and normal ECGs. Despite abnormal myocardial perfusion imaging in more than one in five patients, cardiac outcomes were essentially equal (and very low) in screened versus unscreened patients.

In all patients with diabetes, cardiovascular risk factors should be assessed at least annually. These risk factors include dyslipidemia, hypertension, smoking, a positive family history of premature coronary disease, and the presence of microor macroalbuminuria. Abnormal risk factors should be treated as described elsewhere in these guidelines. Patients at increased CHD risk should receive aspirin and a statin and ACE inhibitor or ARB therapy if hypertensive, unless there are contraindications to a particular drug class. While clear benefit exists for ACE inhibitor and ARB therapy in patients 
with nephropathy or hypertension, the benefits in patients with CVD in the absence of these conditions is less clear, especially when LDL cholesterol is concomitantly controlled $(224,225)$

\section{B. Nephropathy screening and treatment}

\section{Recommendations}

\section{General recommendations}

- To reduce the risk or slow the progression of nephropathy, optimize glucose control. (A)

- To reduce the risk or slow the progression of nephropathy, optimize blood pressure control. (A)

\section{Screening}

- Perform an annual test to assess urine albumin excretion in type 1 diabetic patients with diabetes duration of $\geq 5$ years and in all type 2 diabetic patients, starting at diagnosis. (E)

- Measure serum creatinine at least annually in all adults with diabetes regardless of the degree of urine albumin excretion. The serum creatinine should be used to estimate GFR and stage the level of chronic kidney disease (CKD), if present. (E)

\section{Treatment}

- In the treatment of the nonpregnant patient with micro- or macroalbuminuria, either ACE inhibitors or ARBs should be used. (A)

- While there are no adequate head-tohead comparisons of ACE inhibitors and ARBs, there is clinical trial support for each of the following statements:

- In patients with type 1 diabetes, hypertension, and any degree of albuminuria, ACE inhibitors have been shown to delay the progression of nephropathy. (A)

- In patients with type 2 diabetes, hypertension, and microalbuminuria, both ACE inhibitors and ARBs have been shown to delay the progression to macroalbuminuria. (A)

- In patients with type 2 diabetes, hypertension, macroalbuminuria, and renal insufficiency (serum creatinine $>1.5 \mathrm{mg} / \mathrm{dl}$ ), ARBs have been shown to delay the progression of nephropathy. (A)

- If one class is not tolerated, the other should be substituted. (E)
- Reduction of protein intake to $0.8-1.0$ $\mathrm{g} \cdot \mathrm{kg}$ body $\mathrm{wt}^{-1} \cdot$ day $^{-1}$ in individuals with diabetes and the earlier stages of $\mathrm{CKD}$ and to $0.8 \mathrm{~g} \cdot \mathrm{kg}$ body $\mathrm{wt}^{-1}$. day $^{-1}$ in the later stages of CKD may improve measures of renal function (urine albumin excretion rate, GFR) and is recommended. (B)

- When ACE inhibitors, ARBs, or diuretics are used, monitor serum creatinine and potassium levels for the development of acute kidney disease and hyperkalemia. (E)

- Continued monitoring of urine albumin excretion to assess both response to therapy and progression of disease is recommended. (E)

- Consider referral to a physician experienced in the care of kidney disease when there is uncertainty about the etiology of kidney disease (active urine sediment, absence of retinopathy, rapid decline in GFR), difficult management issues, or advanced kidney disease. (B)

Diabetic nephropathy occurs in 20-40\% of patients with diabetes and is the single leading cause of end-stage renal disease (ESRD). Persistent albuminuria in the range of 30-299 $\mathrm{mg} / 24 \mathrm{~h}$ (microalbuminuria) has been shown to be the earliest stage of diabetic nephropathy in type 1 diabetes and a marker for development of nephropathy in type 2 diabetes. Microalbuminuria is also a well-established marker of increased CVD risk $(226,227)$. Patients with microalbuminuria who progress to macroalbuminuria (300 $\mathrm{mg} / 24 \mathrm{~h}$ ) are likely to progress to ESRD $(228,229)$. However, a number of interventions have been demonstrated to reduce the risk and slow the progression of renal disease.

Intensive diabetes management with the goal of achieving near normoglycemia has been shown in large prospective randomized studies to delay the onset of microalbuminuria and the progression of micro- to macroalbuminuria in patients with type $1(230,231)$ and type $2(49,50)$ diabetes. The UKPDS provided strong evidence that control of blood pressure can reduce the development of nephropathy (163). In addition, large prospective randomized studies in patients with type 1 diabetes have demonstrated that achievement of lower levels of systolic blood pressure $(<140 \mathrm{mmHg})$ resulting from treatment using ACE inhibitors provides a selective benefit over other antihypertensive drug classes in delaying the progression from micro- to macroalbuminuria and can slow the decline in GFR in patients with macroalbuminuria $(180,181,232)$. In type 2 diabetes with hypertension and normoalbuminuria, ACE inhibition has been demonstrated to delay progression to microalbuminuria (233).

In addition, ACE inhibitors have been shown to reduce major CVD outcomes (i.e., myocardial infarction, stroke, death) in patients with diabetes (174), thus further supporting the use of these agents in patients with microalbuminuria, a CVD risk factor. ARBs have also been shown to reduce the rate of progression from micro- to macroalbuminuria as well as ESRD in patients with type 2 diabetes (234236). Some evidence suggests that ARBs have a smaller magnitude of rise in potassium compared with ACE inhibitors in people with nephropathy $(237,238)$. It is important to note that the benefits of both ACE inhibitors and ARBs in those with diabetic nephropathy are strongly associated with the reduction in albuminuria. Combinations of drugs that block the rennin-angiotensin-aldosterone system (e.g., an ACE inhibitor plus an ARB, a mineralocorticoid antagonist, or a direct renin inhibitor) have been shown to provide additional lowering of albuminuria (239242). However, the long-term effects of such combinations on renal or cardiovascular outcomes have not yet been evaluated in clinical trials.

Other drugs, such as diuretics, calcium channel blockers, and $\beta$-blockers, should be used as additional therapy to further lower blood pressure in patients already treated with ACE inhibitors or ARBs (179) or as alternate therapy in the rare individual unable to tolerate ACE inhibitors or ARBs.

Studies in patients with varying stages of nephropathy have shown that protein restriction helps slow the progression of albuminuria, GFR decline, and occurrence of ESRD (243-246). Protein restriction should be considered particularly in patients whose nephropathy seems to be progressing despite optimal glucose and blood pressure control and use of ACE inhibitor and/or ARBs (246).

\section{Assessment of albuminuria status and renal function}

Screening for microalbuminuria can be performed by measurement of the albumin-to-creatinine ratio in a random spot collection (preferred method); 24-h or timed collections are more burdensome and add little to prediction or accuracy 
Table 12-Definitions of abnormalities in albumin excretion

Category Spot collection ( $\mu \mathrm{g} / \mathrm{mg}$ creatinine)

Normal

Microalbuminuria

$<30$

Macro (clinical)-albuminuria

30-299

$\geq 300$
$(247,248)$. Measurement of a spot urine for albumin only, whether by immunoassay or by using a dipstick test specific for microalbumin, without simultaneously measuring urine creatinine, is somewhat less expensive but susceptible to falsenegative and -positive determinations as a result of variation in urine concentration due to hydration and other factors.

Abnormalities of albumin excretion are defined in Table 12. Because of variability in urinary albumin excretion, two of three specimens collected within a 3- to 6-month period should be abnormal before considering a patient to have crossed one of these diagnostic thresholds. Exercise within $24 \mathrm{~h}$, infection, fever, CHF, marked hyperglycemia, and marked hypertension may elevate urinary albumin excretion over baseline values.

Information on presence of abnormal urine albumin excretion in addition to level of GFR may be used to stage CKD. The National Kidney Foundation classification (Table 13) is primarily based on GFR levels and therefore differs from other systems, in which staging is based primarily on urinary albumin excretion (249). Studies have found decreased GFR in the absence of increased urine albumin excretion in a substantial percentage of adults with diabetes $(250,251)$. Epidemiologic evidence suggests that a substantial fraction of those with CKD in the setting of diabetes have little or no detectable albuminuria (250). Serum creatinine should therefore be measured at least annually in all adults with diabetes, regardless of the degree of urine albumin excretion.

Serum creatinine should be used to estimate GFR and to stage the level of CKD, if present. GFR can be estimated using formulae such as the CockroftGault equation or a prediction formula using data from the Modification of Diet and Renal Disease study (252). GFR calculators are available at http://www. nkdep.nih.gov. Many clinical laboratories now report estimated GFR in addition to serum creatinine.

The role of continued annual quantitative assessment of albumin excretion af- ter diagnosis of microalbuminuria and institution of ACE inhibitor or ARB therapy and blood pressure control is unclear. Continued surveillance can assess both response to therapy and progression of disease. Some suggest that reducing abnormal albuminuria ( $>30 \mathrm{mg} / \mathrm{g}$ ) to the normal or near-normal range may improve renal and cardiovascular prognosis, but this approach has not been formally evaluated in prospective trials.

Complications of kidney disease correlate with level of kidney function. When the estimated GFR is $<60 \mathrm{ml} / \mathrm{min}$ per $1.73 \mathrm{~m}^{2}$, screening for anemia, malnutrition, and metabolic bone disease is indicated. Early vaccination against hepatitis $\mathrm{B}$ is indicated in patients likely to progress to end-stage kidney disease.

Consider referral to a physician experienced in the care of kidney disease when there is uncertainty about the etiology of kidney disease (active urine sediment, absence of retinopathy, rapid decline in GFR), difficult management issues, or advanced kidney disease. The threshold for referral may vary depending on the frequency with which a provider encounters diabetic patients with significant kidney disease. Consultation with a nephrologist when stage 4 CKD develops has been found to reduce cost, improve quality of care, and keep people off dialysis longer $(253,254)$. However, nonrenal specialists should not delay educating their patients about the progressive nature of diabetic kidney disease, the renal preservation benefits of aggressive treatment of blood pres- sure, blood glucose, and hyperlipidemia, and the potential need for renal replacement therapy.

\section{Retinopathy screening and treatment}

\section{Recommendations}

\section{General recommendations}

- To reduce the risk or slow the progression of retinopathy, optimize glycemic control. (A)

- To reduce the risk or slow the progression of retinopathy, optimize blood pressure control. (A)

\section{Screening}

- Adults and children aged 10 years or older with type 1 diabetes should have an initial dilated and comprehensive eye examination by an ophthalmologist or optometrist within 5 years after the onset of diabetes. (B)

- Patients with type 2 diabetes should have an initial dilated and comprehensive eye examination by an ophthalmologist or optometrist shortly after the diagnosis of diabetes. (B)

- Subsequent examinations for type 1 and type 2 diabetic patients should be repeated annually by an ophthalmologist or optometrist. Less frequent exams (every 2-3 years) may be considered following one or more normal eye exams. Examinations will be required more frequently if retinopathy is progressing. (B)

- Women with preexisting diabetes who are planning pregnancy or who have become pregnant should have a comprehensive eye examination and be counseled on the risk of development and/or progression of diabetic retinopathy. Eye examination should occur in the first trimester with close follow-up throughout pregnancy and 1 year postpartum. (B)
Table 13-Stages of CKD

\begin{tabular}{llc} 
Stage & \multicolumn{1}{c}{ Description } & $\begin{array}{c}\text { GFR (ml/min per } 1.73 \mathrm{~m}^{2} \\
\text { body surface area) }\end{array}$ \\
\hline 1 & Kidney damage* with normal or increased GFR & $\geq 90$ \\
2 & Kidney damage* with mildly decreased GFR & $60-89$ \\
3 & Moderately decreased GFR & $30-59$ \\
4 & Severely decreased GFR & $15-29$ \\
5 & Kidney failure & $<15$ or dialysis \\
\hline
\end{tabular}

*Kidney damage defined as abnormalities on pathologic, urine, blood, or imaging tests. Adapted and reprinted with permission (248). 


\section{Treatment}

- Promptly refer patients with any level of macular edema, severe NPDR, or any PDR to an ophthalmologist who is knowledgeable and experienced in the management and treatment of diabetic retinopathy. (A)

- Laser photocoagulation therapy is indicated to reduce the risk of vision loss in patients with high-risk PDR and clinically significant macular edema and in some cases of severe NPDR. (A)

- The presence of retinopathy is not a contraindication to aspirin therapy for cardioprotection, as this therapy does not increase the risk of retinal hemorrhage. (A)

Diabetic retinopathy is a highly specific vascular complication of both type 1 and type 2 diabetes, with prevalence strongly related to the duration of diabetes. Diabetic retinopathy is the most frequent cause of new cases of blindness among adults aged $20-74$ years. Glaucoma, cataracts, and other disorders of the eye occur earlier and more frequently in people with diabetes.

In addition to duration of diabetes, other factors that increase the risk of, or are associated with, retinopathy include chronic hyperglycemia (255), the presence of nephropathy (256), and hypertension (257). Intensive diabetes management with the goal of achieving near normoglycemia has been shown in large prospective randomized studies to prevent and/or delay the onset and progression of diabetic retinopathy $(45,49,50)$. Lowering blood pressure has been shown to decrease the progression of retinopathy (163). Several case series and a controlled prospective study suggest that pregnancy in type 1 diabetic patients may aggravate retinopathy $(258,259)$; laser photocoagulation surgery can minimize this risk (259).

One of the main motivations for screening for diabetic retinopathy is the established efficacy of laser photocoagulation surgery in preventing vision loss. Two large trials, the Diabetic Retinopathy Study (DRS) and the Early Treatment Diabetic Retinopathy Study (ETDRS), provide the strongest support for the therapeutic benefits of photocoagulation surgery.

The DRS (260) showed that panretinal photocoagulation surgery reduced the risk of severe vision loss from PDR from $15.9 \%$ in untreated eyes to $6.4 \%$ in treated eyes. The benefit was greatest among patients whose baseline evaluation revealed high-risk characteristics (chiefly disc neovascularization or vitreous hemorrhage). Given the risks of modest loss of visual acuity and contraction of the visual field from panretinal laser surgery, such therapy is primarily recommended for eyes with PDR approaching or having high-risk characteristics.

The ETDRS (261) established the benefit of focal laser photocoagulation surgery in eyes with macular edema, particularly those with clinically significant macular edema, with reduction of doubling of the visual angle (e.g., 20/50 to $20 / 100$ ) from $20 \%$ in untreated eyes to $8 \%$ in treated eyes. The ETDRS also verified the benefits of panretinal photocoagulation for high-risk PDR, but not for mild or moderate NPDR. In older-onset patients with severe NPDR or less-thanhigh-risk PDR, the risk of severe vision loss or vitrectomy was reduced $\sim 50 \%$ by early laser photocoagulation surgery at these stages.

Laser photocoagulation surgery in both trials was beneficial in reducing the risk of further vision loss, but generally not beneficial in reversing already diminished acuity. This preventive effect and the fact that patients with PDR or macular edema may be asymptomatic provide strong support for a screening program to detect diabetic retinopathy.

As retinopathy is estimated to take at least 5 years to develop after the onset of hyperglycemia (262), patients with type 1 diabetes should have an initial dilated and comprehensive eye examination within 5 years after the onset of diabetes. Patients with type 2 diabetes, who generally have had years of undiagnosed diabetes (263) and who have a significant risk of prevalent diabetic retinopathy at time of diabetes diagnosis, should have an initial dilated and comprehensive eye examination soon after diagnosis. Examinations should be performed by an ophthalmologist or optometrist who is knowledgeable and experienced in diagnosing the presence of diabetic retinopathy and is aware of its management. Subsequent examinations for type 1 and type 2 diabetic patients are generally repeated annually. Less frequent exams (every 2-3 years) may be cost effective after one or more normal eye exams (264-266), while examinations will be required more frequently if retinopathy is progressing.

Examinations can also be done with retinal photographs (with or without dilation of the pupil) read by experienced experts. In-person exams are still necessary when the photos are unacceptable and for follow-up of abnormalities detected. This technology has great potential in areas where qualified eye care professionals are not available and may enhance efficiency and reduce costs when the expertise of ophthalmologists can be utilized for more complex examinations and for therapy (267).

Results of eye examinations should be documented and transmitted to the referring health care professional. For a detailed review of the evidence and further discussion of diabetic retinopathy, see the ADA's technical review and position statement on this subject $(268,269)$.

\section{Neuropathy screening and treatment (270)}

\section{Recommendations}

- All patients should be screened for distal symmetric polyneuropathy (DPN) at diagnosis and at least annually thereafter using simple clinical tests. (B)

- Electrophysiological testing is rarely needed, except in situations where the clinical features are atypical. (E)

- Screening for signs and symptoms of cardiovascular autonomic neuropathy should be instituted at diagnosis of type 2 diabetes and 5 years after the diagnosis of type 1 diabetes. Special testing is rarely needed and may not affect management or outcomes. (E)

- Medications for the relief of specific symptoms related to DPN and autonomic neuropathy are recommended, as they improve the quality of life of the patient. (E)

The diabetic neuropathies are heterogeneous with diverse clinical manifestations. They may be focal or diffuse. Most common among the neuropathies are chronic sensorimotor DPN and autonomic neuropathy. Although DPN is a diagnosis of exclusion, complex investigations to exclude other conditions are rarely needed.

The early recognition and appropriate management of neuropathy in the patient with diabetes is important for a number of reasons: 1) nondiabetic neuropathies may be present in patients with diabetes and may be treatable; 2) a number of treatment options exist for symptomatic diabetic neuropathy; 3) up to $50 \%$ of DPN may be asymptomatic, and patients are at risk of insensate injury to their feet; 4) autonomic neuropathy may 
Table 14-Table of drugs to treat symptomatic DPN

\begin{tabular}{lll} 
Class & \multicolumn{1}{c}{ Examples } & \multicolumn{1}{c}{ Typical doses* } \\
\hline Tricyclic drugs & Amitriptyline & 10-75 mg at bedtime \\
& Nortriptyline & 25-75 mg at bedtime \\
& Imipramine & 25-75 mg at bedtime \\
Anticonvulsants & Gabapentin & $300-1,200 \mathrm{mg}$ t.i.d. \\
& Carbamazepine & 200-400 mg t.i.d. \\
& Pregabalin ${ }^{\dagger}$ & $100 \mathrm{mg}$ t.i.d. \\
5-hydroxytryptamine and & Duloxetine ${ }^{\dagger}$ & $60-120 \mathrm{mg}$ daily \\
norepinephrine uptake & & \\
inhibitor & & \\
Substance P inhibitor & Capsaicin cream & $0.025-0.075 \%$ applied t.i.d. or q.i.d.
\end{tabular}

*Dose response may vary; initial doses should be low and titrated up. †Has FDA indication for treatment of painful diabetic neuropathy.

involve every system in the body; and 5) cardiovascular autonomic neuropathy causes substantial morbidity and mortality. Specific treatment for the underlying nerve damage is currently not available, other than improved glycemic control, which may slow progression but not reverse neuronal loss. Effective symptomatic treatments are available for some manifestations of DPN and autonomic neuropathy.

\section{Diagnosis of neuropathy}

Distal symmetric polyneuropathy

Patients with diabetes should be screened annually for DPN using tests such as pinprick sensation, vibration perception (using a 128-Hz tuning fork), 10-g monofilament pressure sensation at the distal plantar aspect of both great toes and metatarsal joints, and assessment of ankle reflexes. Combinations of more than one test have $>87 \%$ sensitivity in detecting DPN. Loss of 10-g monofilament perception and reduced vibration perception predict foot ulcers (270).

Diabetic autonomic neuropathy (271) The symptoms and signs of autonomic dysfunction should be elicited carefully during the history and physical examination. Major clinical manifestations of diabetic autonomic neuropathy include resting tachycardia, exercise intolerance, orthostatic hypotension, constipation, gastroparesis, erectile dysfunction, sudomotor dysfunction, impaired neurovascular function, "brittle diabetes," and hypoglycemic autonomic failure.

Cardiovascular autonomic neuropathy, a CVD risk factor (93), is the most studied and clinically important form of diabetic autonomic neuropathy. Cardiovascular autonomic neuropathy may be indicated by resting tachycardia $(>100$ $\mathrm{bpm}$ ), orthostasis (a fall in systolic blood pressure $>20 \mathrm{mmHg}$ upon standing without an appropriate heart rate response), or other disturbances in autonomic nervous system function involving the skin, pupils, or gastrointestinal and genitourinary systems.

Gastrointestinal neuropathies (e.g., esophageal enteropathy, gastroparesis, constipation, diarrhea, fecal incontinence) are common, and any section of the gastrointestinal tract may be affected. Gastroparesis should be suspected in individuals with erratic glucose control or with upper gastrointestinal symptoms without other identified cause. Evaluation of solid-phase gastric emptying using double-isotope scintigraphy may be done if symptoms are suggestive, but test results often correlate poorly with symptoms. Constipation is the most common lower-gastrointestinal symptom but can alternate with episodes of diarrhea.

Diabetic autonomic neuropathy is also associated with genitourinary tract disturbances. In men, diabetic autonomic neuropathy may cause erectile dysfunction and/or retrograde ejaculation. Evaluation of bladder dysfunction should be performed for individuals with diabetes who have recurrent urinary tract infections, pyelonephritis, incontinence, or a palpable bladder.

\section{Symptomatic treatments \\ DPN}

The first step in management of patients with DPN should be to aim for stable and optimal glycemic control. Although controlled trial evidence is lacking, several observational studies suggest that neuropathic symptoms improve not only with optimization of control, but also with the avoidance of extreme blood glucose fluctuations. Patients with painful DPN may benefit from pharmacological treatment of their symptoms: many agents have efficacy confirmed in published randomized controlled trials, with several FDAapproved for the management of painful DPN. See Table 14 for examples of agents to treat DPN pain.

Treatment of autonomic neuropathy Gastroparesis symptoms may improve with dietary changes and prokinetic agents such as metoclopramide or erythromycin. Treatments for erectile dysfunction may include phosphodiesterase type 5 inhibitors, intracorporeal or intraurethral prostaglandins, vacuum devices, or penile prostheses. Interventions for other manifestations of autonomic neuropathy are described in the ADA statement on neuropathy (270). As with DPN treatments, these interventions do not change the underlying pathology and natural history of the disease process but may have a positive impact on the quality of life of the patient.

\section{E. Foot care}

\section{Recommendations}

- For all patients with diabetes, perform an annual comprehensive foot examination to identify risk factors predictive of ulcers and amputations. The foot examination should include inspection, assessment of foot pulses, and testing for loss of protective sensation (10-g monofilament plus testing any one of the following: vibration using 128-Hz tuning fork, pinprick sensation, ankle reflexes, or vibration perception threshold). (B)

- Provide general foot self-care education to all patients with diabetes. (B)

- A multidisciplinary approach is recommended for individuals with foot ulcers and high-risk feet, especially those with a history of prior ulcer or amputation. (B)

- Refer patients who smoke, have loss of protective sensation and structural abnormalities, or have history of prior lower-extremity complications to foot care specialists for ongoing preventive care and life-long surveillance. (C)

- Initial screening for peripheral arterial disease (PAD) should include a history for claudication and an assessment of the pedal pulses. Consider obtaining an 
ankle-brachial index (ABI), as many patients with PAD are asymptomatic. (C)

- Refer patients with significant claudication or a positive ABI for further vascular assessment and consider exercise, medications, and surgical options. (C)

Amputation and foot ulceration, consequences of diabetic neuropathy and/or $\mathrm{PAD}$, are common and major causes of morbidity and disability in people with diabetes. Early recognition and management of risk factors can prevent or delay adverse outcomes.

The risk of ulcers or amputations is increased in people who have the following risk factors:

- previous amputation

- past foot ulcer history

- peripheral neuropathy

- foot deformity

- peripheral vascular disease

- vision impairment

- diabetic nephropathy (especially patients on dialysis)

- poor glycemic control

- cigarette smoking

Many studies have been published proposing a range of tests that might usefully identify patients at risk of foot ulceration, creating confusion among practitioners as to which screening tests should be adopted in clinical practice. An ADA task force was therefore assembled in 2008 to concisely summarize recent literature in this area and then recommend what should be included in the comprehensive foot exam for adult patients with diabetes. Their recommendations are summarized below, but clinicians should refer to the task force report (272) for further details and practical descriptions of how to perform components of the comprehensive foot examination.

At least annually, all adults with diabetes should undergo a comprehensive foot examination to identify high-risk conditions. Clinicians should ask about history of previous foot ulceration or amputation, neuropathic or peripheral vascular symptoms, impaired vision, tobacco use, and foot care practices. A general inspection of skin integrity and musculoskeletal deformities should be done in a well-lit room. Vascular assessment should include inspection and assessment of pedal pulses.

The neurologic exam recommended is designed to identify loss of protective sensation (LOPS) rather than early neu- ropathy. The clinical examination to identify LOPS is simple and requires no expensive equipment. Five simple clinical tests (use of a 10-g monofilament, vibration testing using a 128- $\mathrm{Hz}$ tuning fork, tests of pinprick sensation, ankle reflex assessment, and testing vibration perception threshold with a biothesiometer), each with evidence from well-conducted prospective clinical cohort studies, are considered useful in the diagnosis of LOPS in the diabetic foot. The task force agrees that any of the five tests listed could be used by clinicians to identify LOPS, although ideally two of these should be regularly performed during the screening exam-normally the 10-g monofilament and one other test. One or more abnormal tests would suggest LOPS, while at least two normal tests (and no abnormal test) would rule out LOPS. The last test listed, vibration assessment using a biothesiometer or similar instrument, is widely used in the U.S.; however, identification of the patient with LOPS can easily be carried out without this or other expensive equipment.

Initial screening for PAD should include a history for claudication and an assessment of the pedal pulses. A diagnostic ABI should be performed in any patient with symptoms of PAD. Due to the high estimated prevalence of PAD in patients with diabetes and the fact that many patients with PAD are asymptomatic, an ADA consensus statement on PAD (273) suggested that a screening $A B I$ be performed in patients over 50 years of age and be considered in patients under 50 years of age who have other PAD risk factors (e.g., smoking, hypertension, hyperlipidemia, or duration of diabetes $>10$ years). Refer patients with significant symptoms or a positive ABI for further vascular assessment and consider exercise, medications, and surgical options (273)

Patients with diabetes and high-risk foot conditions should be educated regarding their risk factors and appropriate management. Patients at risk should understand the implications of the loss of protective sensation, the importance of foot monitoring on a daily basis, the proper care of the foot, including nail and skin care, and the selection of appropriate footwear. Patients with loss of protective sensation should be educated on ways to substitute other sensory modalities (hand palpation, visual inspection) for surveillance of early foot problems. The patient's understanding of these issues and their physical ability to conduct proper foot surveillance and care should be assessed. Patients with visual difficulties, physical constraints preventing movement, or cognitive problems that impair their ability to assess the condition of the foot and to institute appropriate responses will need other people, such as family members, to assist in their care.

People with neuropathy or evidence of increased plantar pressure (e.g., erythema, warmth, callus, or measured pressure) may be adequately managed with well-fitted walking shoes or athletic shoes that cushion the feet and redistribute pressure. Callus can be debrided with a scalpel by a foot care specialist or other health professional with experience and training in foot care. People with bony deformities (e.g., hammertoes, prominent metatarsal heads, bunions) may need extrawide or -depth shoes. People with extreme bony deformities (e.g., Charcot foot) who cannot be accommodated with commercial therapeutic footwear may need custom-molded shoes.

Foot ulcers and wound care may require care by a podiatrist, orthopedic or vascular surgeon, or rehabilitation specialist experienced in the management of individuals with diabetes. For a complete discussion, see the ADA's consensus statement on diabetic foot wound care (274).

\section{DIABETES CARE IN SPECIFIC POPULATIONS}

\section{A. Children and adolescents}

\section{Type 1 diabetes}

Three-quarters of all cases of type 1 diabetes are diagnosed in individuals $<18$ years of age. Because children are not simply "small adults," it is appropriate to consider the unique aspects of care and management of children and adolescents with type 1 diabetes. Children with diabetes differ from adults in many respects, including changes in insulin sensitivity related to sexual maturity and physical growth, ability to provide self-care, supervision in child care and school, and unique neurologic vulnerability to hypoglycemia and DKA. Attention to such issues as family dynamics, developmental stages, and physiologic differences related to sexual maturity are all essential in developing and implementing an optimal diabetes regimen. Although recommendations for children and adolescents are 


\begin{tabular}{|c|c|c|c|c|}
\hline \multirow[b]{2}{*}{ Values by age (years) } & \multicolumn{2}{|c|}{$\begin{array}{l}\text { Plasma blood glucose goal range } \\
\text { (mg/dl) }\end{array}$} & \multirow[b]{2}{*}{ A $1 C$} & \multirow[b]{2}{*}{ Rationale } \\
\hline & Before meals & Bedtime/overnight & & \\
\hline Toddlers and preschoolers (0-6) & $100-180$ & $110-200$ & $<8.5 \%($ but $>7.5 \%)$ & $\begin{array}{l}\text { High risk and vulnerability to } \\
\text { hypoglycemia }\end{array}$ \\
\hline School age (6-12) & $90-180$ & $100-180$ & $<8 \%$ & $\begin{array}{l}\text { Risks of hypoglycemia and relatively } \\
\text { low risk of complications prior to } \\
\text { puberty }\end{array}$ \\
\hline Adolescents and young adults (13-19) & $90-130$ & $90-150$ & $<7.5 \%$ & $\begin{array}{l}\text { - Risk of severe hypoglycemia } \\
\text { - Developmental and psychological } \\
\text { issues } \\
\text { - A lower goal }(<7.0 \%) \text { is } \\
\text { reasonable if it can be achieved } \\
\text { without excessive hypoglycemia }\end{array}$ \\
\hline
\end{tabular}

Key concepts in setting glycemic goals:

- Goals should be individualized and lower goals may be reasonable based on benefit-risk assessment.

- Blood glucose goals should be higher than those listed above in children with frequent hypoglycemia or hypoglycemia unawareness.

- Postprandial blood glucose values should be measured when there is a discrepancy between pre-prandial blood glucose values and AlC levels and to help assess glycemia in those on basal/bolus regimens.

less likely to be based on clinical trial evidence, because of current and historical restraints placed on conducting research in children, expert opinion and a review of available and relevant experimental data are summarized in the ADA statement on care of children and adolescents with type 1 diabetes (275).

Ideally, the care of a child or adolescent with type 1 diabetes should be provided by a multidisciplinary team of specialists trained in the care of children with pediatric diabetes. At the very least, education of the child and family should be provided by health care providers trained and experienced in childhood diabetes and sensitive to the challenges posed by diabetes in this age-group. At the time of initial diagnosis, it is essential that diabetes education be provided in a timely fashion, with the expectation that the balance between adult supervision and self-care should be defined by, and will evolve according to, physical, psychological, and emotional maturity. MNT should be provided at diagnosis, and at least annually thereafter, by an individual experienced with the nutritional needs of the growing child and the behavioral issues that have an impact on adolescent diets, including risk for disordered eating.

\section{a. Glycemic control}

\section{Recommendations}

- Consider age when setting glycemic goals in children and adolescents with type 1 diabetes, with less stringent goals for younger children. (E)
While current standards for diabetes management reflect the need to maintain glucose control as near to normal as safely possible, special consideration must be given to the unique risks of hypoglycemia in young children. Glycemic goals need to be modified to take into account the fact that most children $<6$ or 7 years of age have a form of hypoglycemic unawareness. Their counterregulatory mechanisms are immature and they may lack the cognitive capacity to recognize and respond to hypoglycemic symptoms, placing them at greater risk for severe hypoglycemia and its sequelae. In addition, and unlike the case in adults, young children below the age of 5 years are at risk for permanent cognitive impairment after episodes of severe hypoglycemia (276-278). Extensive evidence indicates that near normalization of blood glucose levels is seldom attainable in children and adolescents after the honeymoon (remission) period. The AlC level achieved in the "intensive" adolescent cohort of the DCCT group was $>1 \%$ higher than that achieved by adult DCCT subjects and above current ADA recommendations for patients in general. However, the increased frequency of use of basal bolus regimens (including insulin pumps) in youth from infancy through adolescence has been associated with more children reaching ADA blood glucose targets $(279,280)$ in those families in which both parents and the child with diabetes are motivated to perform the required diabetes-related tasks.

In selecting glycemic goals, the bene- fits on long-term health outcomes of achieving a lower AlC must be weighed against the unique risks of hypoglycemia and the difficulties achieving near normoglycemia in children and youth. Agespecific glycemic and A1C goals are presented in Table 15.

\section{b. Screening and management of chronic complications in children and adolescents with type 1 diabetes}

\section{i. Nephropathy}

\section{Recommendations}

- Annual screening for microalbuminuria, with a random spot urine sample for microalbumin-to-creatinine ratio, should be initiated once the child is 10 years of age and has had diabetes for 5 years. (E)

- Confirmed, persistently elevated microalbumin levels on two additional urine specimens should be treated with an ACE inhibitor, titrated to normalization of microalbumin excretion if possible. (E)

\section{ii. Hypertension}

\section{Recommendations}

- Treatment of high-normal blood pressure (systolic or diastolic blood pressure consistently between the 90-95th percentile for age, sex, and height) should include dietary intervention and exercise aimed at weight control and increased physical activity, if appropriate. If target blood pressure is not 
reached with 6-12 months of lifestyle intervention, pharmacologic treatment should be initiated. (E)

- Pharmacologic treatment of high blood pressure (systolic or diastolic blood pressure consistently above the 95th percentile for age, sex, and height or consistently $>130 / 80 \mathrm{mmHg}$ for adolescents) should be initiated along with lifestyle intervention as soon as the diagnosis is confirmed. (E)

- ACE inhibitors should be considered for the initial treatment of hypertension. (E)

- The goal of treatment is a blood pressure consistently $<130 / 80$ or below the 90th percentile for age, sex, and height, whichever is lower. (E)

Hypertension in childhood is defined as an average systolic or diastolic blood pressure 95th percentile for age, sex, and height percentile measured on at least 3 separate days. "High-normal" blood pressure is defined as an average systolic or diastolic blood pressure 90th but $<95$ th percentile for age, sex, and height percentile measured on at least 3 separate days. Normal blood pressure levels for age, sex, and height and appropriate methods for determinations are available online at www.nhlbi.nih.gov/health/prof/heart/ hbp/hbp_ped.pdf.

\section{iii. Dyslipidemia}

\section{Recommendations}

\section{Screening}

- If there is a family history of hypercholesterolemia (total cholesterol >240 $\mathrm{mg} / \mathrm{dl}$ ) or a cardiovascular event before age 55 years, or if family history is unknown, then a fasting lipid profile should be performed on children $>2$ years of age soon after diagnosis (after glucose control has been established). If family history is not of concern, then the first lipid screening should be performed at puberty ( $\geq 10$ years). All children diagnosed with diabetes at or after puberty should have a fasting lipid profile performed soon after diagnosis (after glucose control has been established). (E)

- For both age-groups, if lipids are abnormal, annual monitoring is recommended. If LDL cholesterol values are within the accepted risk levels $(<100$ $\mathrm{mg} / \mathrm{dl}$ [2.6 $\mathrm{mmol} / \mathrm{l}]$ ), a lipid profile should be repeated every 5 years. (E)

\section{Treatment}

- Initial therapy should consist of optimization of glucose control and MNT using a Step 2 AHA diet aimed at a decrease in the amount of saturated fat in the diet. (E)

- After the age of 10, the addition of a statin is recommended in patients who, after MNT and lifestyle changes, have LDL $>160 \mathrm{mg} / \mathrm{dl}(4.1 \mathrm{mmol} / \mathrm{l})$ or LDL cholesterol $>130 \mathrm{mg} / \mathrm{dl}(3.4 \mathrm{mmol} / \mathrm{l})$ and one or more CVD risk factors. (E)

- The goal of therapy is an LDL cholesterol value $<100 \mathrm{mg} / \mathrm{dl}(2.6 \mathrm{mmol} / \mathrm{l})$. (E)

People diagnosed with type 1 diabetes in childhood have a high risk of early subclinical (281-283) and clinical (284) CVD. Although intervention data are lacking, the AHA categorizes type 1 children in the highest tier for cardiovascular risk and recommends both lifestyle and pharmacologic treatment for those with elevated LDL cholesterol levels $(285,286)$. Initial therapy should be with a Step 2 AHA diet, which restricts saturated fat to $7 \%$ of total calories and restricts dietary cholesterol to $200 \mathrm{mg}$ per day. Data from randomized clinical trials in children as young as 7 months of age indicate that this diet is safe and does not interfere with normal growth and development $(287,288)$.

For children over the age of 10 with persistent elevation of LDL cholesterol despite lifestyle therapy, statins should be considered. Neither long-term safety nor cardiovascular outcome efficacy has been established for children. However, recent studies have shown short-term safety equivalent to that seen in adults and efficacy in lowering LDL cholesterol levels, improving endothelial function, and causing regression of carotid intimal thickening (289-291). No statin is approved for use under the age of 10 , and statin treatment should generally not be used in type 1 children before this age.

\section{iv. Retinopathy}

\section{Recommendations}

- The first ophthalmologic examination should be obtained once the child is $\geq 10$ years of age and has had diabetes for 3-5 years. (E)

- After the initial examination, annual routine follow-up is generally recommended. Less frequent examinations may be acceptable on the advice of an eye care professional. (E)
Although retinopathy most commonly occurs after the onset of puberty and after 5-10 years of diabetes duration, it has been reported in prepubertal children and with diabetes duration of only 1-2 years. Referrals should be made to eye care professionals with expertise in diabetic retinopathy, an understanding of the risk for retinopathy in the pediatric population, and experience in counseling the pediatric patient and family on the importance of early prevention/ intervention.

\section{v. Celiac disease}

\section{Recommendations}

- Patients with type 1 diabetes should be screened for celiac disease by measuring tissue transglutaminase or antiendomysial antibodies, with documentation of normal serum IgA levels, soon after the diagnosis of diabetes. (E)

- Testing should be repeated if growth failure, failure to gain weight, weight loss, or gastroenterologic symptoms occur. (E)

- Consideration should be given to periodic re-screening of asymptomatic individuals. (E)

- Children with positive antibodies should be referred to a gastroenterologist for evaluation. (E)

- Children with confirmed celiac disease should have consultation with a dietitian and placed on a gluten-free diet. (E)

Celiac disease is an immune-mediated disorder that occurs with increased frequency in patients with type 1 diabetes (1-16\% of individuals compared with $0.3-1 \%$ in the general population) $(292,293)$. Symptoms of celiac disease include diarrhea, weight loss or poor weight gain, growth failure, abdominal pain, chronic fatigue, malnutrition due to malabsorption, and other gastrointestinal problems and unexplained hypoglycemia or erratic blood glucose concentrations.

\section{vi. Hypothyroidism}

\section{Recommendations}

- Patients with type 1 diabetes should be screened for thyroid peroxidase and thyroglobulin antibodies at diagnosis. (E)

- TSH concentrations should be measured after metabolic control has been established. If normal, they should be rechecked every $1-2$ years, or if the pa- 
tient develops symptoms of thyroid dysfunction, thyromegaly, or an abnormal growth rate. Free T4 should be measured if TSH is abnormal. (E)

Autoimmune thyroid disease is the most common autoimmune disorder associated with diabetes, occurring in 17-30\% of patients with type 1 diabetes (294). The presence of thyroid autoantibodies is predictive of thyroid dysfunction, generally hypothyroidism but less commonly hyperthyroidism (295). Subclinical hypothyroidism may be associated with increased risk of symptomatic hypoglycemia (296) and with reduced linear growth (297). Hyperthyroidism alters glucose metabolism, potentially resulting in deterioration of metabolic control.

c. "Adherence." No matter how sound the medical regimen, it can only be as good as the ability of the family and/or individual to implement it. Family involvement in diabetes remains an important component of optimal diabetes management throughout childhood and into adolescence. Health care providers who care for children and adolescents, therefore, must be capable of evaluating the behavioral, emotional, and psychosocial factors that interfere with implementation and then must work with the individual and family to resolve problems that occur and/or to modify goals as appropriate.

d. School and day care. Since a sizable portion of a child's day is spent in school, close communication with school or day care personnel is essential for optimal diabetes management, safety, and maximal academic opportunities. See Section V.III.B, Diabetes Care in the School and Day Care Setting, for further discussion.

\section{Type 2 diabetes}

The incidence of type 2 diabetes in adolescents is increasing, especially in ethnic minority populations (20). Distinction between type 1 and type 2 diabetes in children can be difficult, since the prevalence of overweight in children continues to rise and since autoantigens and ketosis may be present in a substantial number of patients with features of type 2 diabetes (including obesity and acanthosis nigricans). Such a distinction at the time of diagnosis is critical because treatment regimens, educational approaches, and dietary counsel will differ markedly between the two diagnoses. Because type 2 diabetes has a significant incidence of hypertension, dyslipidemia, and mi- croalbuminuria at diagnosis (298), it is recommended that screening for the comorbidities and complications of diabetes, including fasting lipid profile, microalbuminuria assessment, and dilated eye examinations, begin at the time of diagnosis. The ADA consensus statement on this subject (22) provides guidance on the prevention, screening, and treatment of type 2 diabetes and its comorbidities in young people.

\section{B. Preconception care}

\section{Recommendations}

- AlC levels should be as close to normal as possible $(<7 \%)$ in an individual patient before conception is attempted. (B)

- Starting at puberty, preconception counseling should be incorporated in the routine diabetes clinic visit for all women of child-bearing potential. (C)

- Women with diabetes who are contemplating pregnancy should be evaluated and, if indicated, treated for diabetic retinopathy, nephropathy, neuropathy, and CVD. (E)

- Medications used by such women should be evaluated before conception, since drugs commonly used to treat diabetes and its complications may be contraindicated or not recommended in pregnancy, including statins, ACE inhibitors, ARBs, and most noninsulin therapies. (E)

Major congenital malformations remain the leading cause of mortality and serious morbidity in infants of mothers with type 1 and type 2 diabetes. Observational studies indicate that the risk of malformations increases continuously with increasing maternal glycemia during the first 6-8 weeks of gestation, as defined by firsttrimester AlC concentrations. There is no threshold for AlC values below which risk disappears entirely. However, malformation rates above the $1-2 \%$ background rate of nondiabetic pregnancies appear to be limited to pregnancies in which first-trimester AlC concentrations are $>1 \%$ above the normal range for a nondiabetic pregnant woman.

Preconception care of diabetes appears to reduce the risk of congenital malformations. Five nonrandomized studies compared rates of major malformations in infants between women who participated in preconception diabetes care programs and women who initiated intensive diabetes management after they were already pregnant. The preconception care pro- grams were multidisciplinary and designed to train patients in diabetes selfmanagement with diet, intensified insulin therapy, and SMBG. Goals were set to achieve normal blood glucose concentrations, and $>80 \%$ of subjects achieved normal $\mathrm{AlC}$ concentrations before they became pregnant (299-303). In all five studies, the incidence of major congenital malformations in women who participated in preconception care (range 1.0 $1.7 \%$ of infants) was much lower than the incidence in women who did not participate (range $1.4-10.9 \%$ of infants). One limitation of these studies is that participation in preconception care was selfselected rather than randomized. Thus, it is impossible to be certain that the lower malformation rates resulted fully from improved diabetes care. Nonetheless, the evidence supports the concept that malformations can be reduced or prevented by careful management of diabetes before pregnancy.

Planned pregnancies greatly facilitate preconception diabetes care. Unfortunately, nearly two-thirds of pregnancies in women with diabetes are unplanned, leading to a persistent excess of malformations in infants of diabetic mothers. To minimize the occurrence of these devastating malformations, standard care for all women with diabetes who have childbearing potential, beginning at the onset of puberty or at diagnosis, should include 1) education about the risk of malformations associated with unplanned pregnancies and poor metabolic control and 2) use of effective contraception at all times, unless the patient has good metabolic control and is actively trying to conceive.

Women contemplating pregnancy need to be seen frequently by a multidisciplinary team experienced in the management of diabetes before and during pregnancy. The goals of preconception care are to 1) involve and empower the patient in the management of her diabetes, 2) achieve the lowest AlC test results possible without excessive hypoglycemia, 3) assure effective contraception until stable and acceptable glycemia is achieved, and 4) identify, evaluate, and treat longterm diabetic complications such as retinopathy, nephropathy, neuropathy, hypertension, and CHD.

Among the drugs commonly used in the treatment of patients with diabetes, a number may be relatively or absolutely contraindicated during pregnancy. Statins are category $\mathrm{X}$ (contraindicated for use in pregnancy) and should be discon- 
tinued before conception, as should ACE inhibitors (304). ARBs are category C (risk cannot be ruled out) in the first trimester but category D (positive evidence of risk) in later pregnancy and should generally be discontinued before pregnancy. Among the oral antidiabetic agents, metformin and acarbose are classified as category B (no evidence of risk in humans) and all others as category C. Potential risks and benefits of oral antidiabetic agents in the preconception period must be carefully weighed, recognizing that data are insufficient to establish the safety of these agents in pregnancy.

For further discussion of preconception care, see the ADA's technical review (305) and position statement (306) on this subject.

\section{Older adults}

\section{Recommendations}

- Older adults who are functional, cognitively intact, and have significant life expectancy should receive diabetes treatment using goals developed for younger adults. (E)

- Glycemic goals for older adults not meeting the above criteria may be relaxed using individual criteria, but hyperglycemia leading to symptoms or risk of acute hyperglycemic complications should be avoided in all patients. (E)

- Other cardiovascular risk factors should be treated in older adults with consideration of the time frame of benefit and the individual patient. Treatment of hypertension is indicated in virtually all older adults, and lipid and aspirin therapy may benefit those with life expectancy at least equal to the time frame of primary or secondary prevention trials. (E)

- Screening for diabetes complications should be individualized in older adults, but particular attention should be paid to complications that would lead to functional impairment. (E)

Diabetes is an important health condition for the aging population; at least 20\% of patients over the age of 65 years have diabetes, and this number can be expected to grow rapidly in the coming decades. Older individuals with diabetes have higher rates of premature death, functional disability, and coexisting illnesses such as hypertension, CHD, and stroke than those without diabetes. Older adults with diabetes are also at greater risk than other older adults for several common ge- riatric syndromes, such as polypharmacy, depression, cognitive impairment, urinary incontinence, injurious falls, and persistent pain.

The American Geriatric Society's guidelines for improving the care of the older person with diabetes (307) have influenced the following discussion and recommendations. The care of older adults with diabetes is complicated by their clinical and functional heterogeneity. Some older individuals developed diabetes years earlier and may have significant complications; others who are newly diagnosed may have had years of undiagnosed diabetes with resultant complications or may have few complications from the disease. Some older adults with diabetes are frail and have other underlying chronic conditions, substantial diabetes-related comorbidity, or limited physical or cognitive functioning. Other older individuals with diabetes have little comorbidity and are active. Life expectancies are highly variable for this population, but often longer than clinicians realize. Providers caring for older adults with diabetes must take this heterogeneity into consideration when setting and prioritizing treatment goals.

There are few long-term studies in older adults demonstrating the benefits of intensive glycemic, blood pressure, and lipid control. Patients who can be expected to live long enough to reap the benefits of long-term intensive diabetes management and who are active, have good cognitive function, and are willing to undertake the responsibility of selfmanagement should be encouraged to do so and be treated using the goals for younger adults with diabetes.

For patients with advanced diabetes complications, life-limiting comorbid illness, or substantial cognitive or functional impairment, it is reasonable to set less intensive glycemic target goals. These patients are less likely to benefit from reducing the risk of microvascular complications and more likely to suffer serious adverse effects from hypoglycemia. However, patients with poorly controlled diabetes may be subject to acute complications of diabetes, including dehydration, poor wound healing, and hyperglycemic hyperosmolar coma. Glycemic goals at a minimum should avoid these consequences.

Although control of hyperglycemia may be important in older individuals with diabetes, greater reductions in morbidity and mortality may result from control of other cardiovascular risk factors rather than from tight glycemic control alone. There is strong evidence from clinical trials of the value of treating hypertension in the elderly $(308,309)$. There is less evidence for lipidlowering and aspirin therapy, although the benefits of these interventions for primary and secondary prevention are likely to apply to older adults whose life expectancies equal or exceed the time frames seen in clinical trials.

Special care is required in prescribing and monitoring pharmacologic therapy in older adults. Metformin is often contraindicated because of renal insufficiency or significant heart failure. TZDs can cause fluid retention, which may exacerbate or lead to heart failure. They are contraindicated in patients with CHF (New York Heart Association class III and IV) and if used at all should be used very cautiously in those with, or at risk for, milder degrees of CHF. Sulfonylureas, other insulin secretagogues, and insulin can cause hypoglycemia. Insulin use requires that patients or caregivers have good visual and motor skills and cognitive ability. Drugs should be started at the lowest dose and titrated up gradually until targets are reached or side effects develop.

Screening for diabetes complications in older adults also should be individualized. Particular attention should be paid to complications that can develop over short periods of time and/or that would significantly impair functional status, such as visual and lower-extremity complications.

\section{DIABETES CARE IN SPECIFIC SETTINGS}

\section{A. Diabetes care in the hospital}

\section{Recommendations}

- All patients with diabetes admitted to the hospital should have their diabetes clearly identified in the medical record. (E)

- All patients with diabetes should have an order for blood glucose monitoring, with results available to all members of the health care team. (E)

- Goals for blood glucose levels:

- Critically ill surgical patients' blood glucose levels should be kept as close to $110 \mathrm{mg} / \mathrm{dl}(6.1 \mathrm{mmol} / \mathrm{l})$ as possible and generally $<140 \mathrm{mg} / \mathrm{dl}$ (7.8 $\mathrm{mmol} / \mathrm{l})$. (A) These patients require an intravenous insulin protocol that has demonstrated efficacy and safety in achieving the desired glucose 
range without increasing risk for severe hypoglycemia. (E)

- Critically ill nonsurgical patients' glycemic targets are less well defined. Intravenous insulin infusion protocols targeting blood glucose levels $<110-140 \mathrm{mg} / \mathrm{dl}$ have been shown to reduced morbidity and mortality in some, but not all studies. Intravenous insulin infusion protocols that effectively and safely keep blood glucose $<140 \mathrm{mg} / \mathrm{dl}$ are recommended. (C)

- For non-critically ill patients, there is no clear evidence for specific blood glucose goals. Since cohort data suggest that outcomes are better in hospitalized patients with fasting glucose $<126 \mathrm{mg} / \mathrm{dl}$ and all random glucoses $<180-200$, these goals are reasonable if they can be safely achieved. Insulin is the preferred drug to treat hyperglycemia in most cases. (E)

- Due to concerns regarding the risk of hypoglycemia, some institutions may consider these blood glucose levels to be overly aggressive for initial targets. Through quality improvement, glycemic goals should systematically be reduced to the recommended levels. (E)

- Scheduled prandial insulin doses should be appropriately timed in relation to meals and should be adjusted according to point-of-care glucose levels. The traditional sliding-scale insulin regimens are ineffective as monotherapy and are generally not recommended. (C)

- Using correction dose or "supplemental" insulin to correct premeal hyperglycemia in addition to scheduled prandial and basal insulin is recommended. (E)

- Glucose monitoring with orders for correction insulin should be initiated in any patient not known to be diabetic who receives therapy associated with high risk for hyperglycemia, including high-dose glucocorticoids therapy, initiation of enteral or parenteral nutrition, or other medications such as octreotide or immunosuppressive medications. (B) If hyperglycemia is documented and persistent, initiation of basal/bolus insulin therapy may be necessary. Such patients should be treated to the same glycemic goals as patients with known diabetes. (E)

- A plan for treating hypoglycemia should be established for each patient. Episodes of hypoglycemia in the hospital should be tracked. (E)
- All patients with diabetes admitted to the hospital should have an AlC obtained if the result of testing in the previous 2-3 months is not available. (E)

- A diabetes education plan including "survival skills education" and follow-up should be developed for each patient. (E)

- Patients with hyperglycemia in the hospital who do not have a diagnosis of diabetes should have appropriate plans for follow-up testing and care documented at discharge. (E)

The management of diabetes in the hospital is extensively reviewed in an ADA technical review (310). This review, as well as a consensus statement by the American Association of Clinical Endocrinologists (AACE) with co-sponsorship by $\operatorname{ADA}(311,312)$ and a report of a joint ADA-AACE task force on the topic (313), form the basis for the discussion and guidelines in this section.

The literature on hospitalized patients with hyperglycemia typically describes three categories:

- Medical history of diabetes: diabetes previously diagnosed and acknowledged by the patient's treating physician.

- Unrecognized diabetes: hyperglycemia (fasting blood glucose $126 \mathrm{mg} / \mathrm{dl}$ or random blood glucose $200 \mathrm{mg} / \mathrm{dl}$ ) occurring during hospitalization and confirmed as diabetes after hospitalization by standard diagnostic criteria but unrecognized as diabetes by the treating physician during hospitalization.

- Hospital-related hyperglycemia: hyperglycemia (fasting blood glucose 126 $\mathrm{mg} / \mathrm{dl}$ or random blood glucose $\geq 200$ $\mathrm{mg} / \mathrm{dl}$ ) occurring during the hospitalization that reverts to normal after hospital discharge.

The prevalence of diabetes in hospitalized adult patients is not precisely known. In the year $2000,12.4 \%$ of hospital discharges in the U.S. listed diabetes as a diagnosis, but this is likely an underestimate. The prevalence of diabetes in hospitalized adults is conservatively estimated at $12-25 \%$, depending on the thoroughness used in identifying patients. In the year 2003, there were 5.1 million hospitalizations with diabetes as a listed diagnosis, a 2.3-fold increase over 1980 rates (314).

The management of hyperglycemia in the hospital was traditionally considered secondary in importance to the condition that prompted admission (313).

A rapidly growing body of literature supports targeted glucose control in the hospital setting for potential improved mortality, morbidity, and health economic outcomes. Hyperglycemia in the hospital may result from stress, decompensation of type 1 or type 2 or other forms of diabetes, and/or may be iatrogenic due to withholding of antihyperglycemic medications or administration of hyperglycemia-provoking agents such as glucocorticoids or vasopressors.

\section{In-hospital hyperglycemia and outcomes}

a. General medicine and surgery. Observational studies suggest an association between hyperglycemia and increased mortality. Surgical patients with at least one blood glucose value $>220 \mathrm{mg} / \mathrm{dl}$ (12.2 $\mathrm{mmol} / \mathrm{l}$ ) on the first postoperative day have significantly higher infection rates (315).

When admissions on general medicine and surgery units were studied, patients with new hyperglycemia had significantly increased in-hospital mortality, as did patients with known diabetes. In addition, length of stay was higher for the new hyperglycemic group, and patients in either hyperglycemic group were more likely to require intensive care unit (ICU) care and transitional or nursing home care. Better outcomes were demonstrated in patients with fasting and admission blood glucose $<126 \mathrm{mg} / \mathrm{dl}$ ( 7 $\mathrm{mmol} / \mathrm{l})$ and all random blood glucose levels <200 mg/dl (11.1 mmol/l) (316).

b. CVD and critical care. A significant relationship exists between blood glucose levels and mortality in the setting of acute myocardial infarction. A meta-analysis of 15 studies compared in-hospital mortality in both hyper- and normoglycemic patients with and without diabetes. In subjects without known diabetes whose admission blood glucose averaged 109.8 $\mathrm{mg} / \mathrm{dl}(6.1 \mathrm{mmol} / \mathrm{l})$, the relative risk for in-hospital mortality was increased significantly. When diabetes was present and admission glucose averaged $180 \mathrm{mg} / \mathrm{dl}$ $(10 \mathrm{mmol} / \mathrm{l})$, risk of death was moderately increased compared with patients who had diabetes but less hyperglycemia on admission (317). Another study (318) demonstrated a strong independent relationship between admission blood glucose values and both in-hospital and 1-year mortality; rates were significantly lower in subjects with admission plasma 
glucose $<100.8 \mathrm{mg} / \mathrm{dl}(5.6 \mathrm{mmol} / \mathrm{l})$ than in those with plasma glucose $199.8 \mathrm{mg} / \mathrm{dl}$ (11 mmol/l).

These studies focused on admission blood glucose as a predictor of outcomes, rather than inpatient glycemic management per se. Higher admission plasma glucose levels in patients with a prior history of diabetes could reflect the degree of glycemic control in the outpatient setting, thus linking outpatient glycemic control to outcomes in the inpatient population. In patients without a prior history of diabetes, admission hyperglycemia could represent case finding of patients with previously undiagnosed diabetes, an unmasking of risk in a population at high risk for diabetes or more severe illness at admission.

In the initial Diabetes and InsulinGlucose Infusion in Acute Myocardial Infarction (DIGAMI) study $(319,320)$, insulin-glucose infusion followed by at least 3 months of subcutaneous insulin treatment in diabetic patients with acute myocardial infarction improved longterm survival. Mean blood glucose in the intensive insulin intervention arm was $172.8 \mathrm{mg} / \mathrm{dl}$ (9.6 mmol/l), compared with $210.6 \mathrm{mg} / \mathrm{dl}(11.7 \mathrm{mmol} / \mathrm{l})$ in the "conventional" group. The broad range of blood glucose levels within each arm limits the ability to define specific blood glucose target thresholds.

Three more recent studies (321-323) using an insulin-glucose infusion did not show a reduction in mortality in the intervention groups. However, in each of these studies, blood glucose levels were positively correlated with mortality. In the Hyperglycemia: Intensive Insulin Infusion In Infarction (HI-5) study, a decrease in both CHF and reinfarction was observed in the group receiving intensive insulin therapy for at least $24 \mathrm{~h}$.

c. Cardiac surgery. Attainment of targeted glucose control in patients with diabetes undergoing cardiac surgery is associated with reduced mortality and risk of deep sternal wound infections $(324,325)$. Although these studies used historical controls and were not randomized, they support the concept that perioperative hyperglycemia is an independent predictor of infection in patients with diabetes (326), with the lowest mortality in patients with blood glucose $<150 \mathrm{mg} / \mathrm{dl}$ (8.3 mmol/l) (327).

d. Critical care. A mixed group of patients with and without diabetes admitted to a surgical ICU were randomized to receive intensive insulin therapy (target blood glucose $80-110 \mathrm{mg} / \mathrm{dl}$ [4.4-6.1 mmol/l]) or conventional therapy. Intensive insulin therapy achieved a mean blood glucose of $103 \mathrm{mg} / \mathrm{dl}(5.7 \mathrm{mmol} / \mathrm{l})$ and was associated with reduced mortality during the ICU stay and decreased overall inhospital mortality (328). Hospital and ICU survival were linearly associated with ICU glucose levels, with the highest survival rates occurring in patients achieving an average blood glucose $<110 \mathrm{mg} / \mathrm{dl}$ (6.1 mmol/l) (329).

A subsequent study of a similar intervention in patients in a medical ICU (330) showed that the group receiving intensive insulin therapy had reduced morbidity but no difference in mortality overall. Death rates were significantly lower in those patients who were treated for longer than 3 days; but these patients could not be identified before therapy. In another study using a similar intervention targeting a blood glucose range of 4.4-6.1 mmol in patients admitted with sepsis, no difference in mortality from the conventionally treated group was observed. There were more episodes of hypoglycemia, defined as a blood glucose $<40$ $\mathrm{mg} / \mathrm{dl}(2.2 \mathrm{mmol} / \mathrm{l})$, and more serious adverse events in the group receiving intensive insulin therapy (331).

While an earlier meta-analysis concluded that insulin therapy in critically ill patients had a beneficial effect on shortterm mortality in different clinical settings (332), a more recent meta-analysis, involving 29 studies and over 8,000 patients, failed to show any mortality benefit from intensive glucose control. Tight glucose control did reduce the relative risk of septicemia by $26 \%$ (333). While this latter meta-analysis investigated strategies with target blood glucose levels of $80-$ $110 \mathrm{mg} / \mathrm{dl}(4.4-6.1 \mathrm{mmol} / \mathrm{l})$, studies with less stringent glucose targets were also included. Stratification by glucose target did not demonstrate any heterogeneity. The authors of this analysis as well as an accompanying editorial both recommend that glycemic targets in critically ill patients be revisited (328-331).

While results from ongoing clinical trials are still pending, it is clear that uncontrolled hyperglycemia is associated with adverse outcomes in critically ill patients and that achieving levels of glucose control below $140 \mathrm{mg} / \mathrm{dl}$ are reasonable, provided that protocols that minimize risk for hypoglycemia are utilized and that personnel are well educated in the direct application of these protocols.

\section{Glycemic targets in hospitalized patients}

There is relatively strong evidence from randomized controlled trials for a glycemic target of blood glucose $<110 \mathrm{mg} / \mathrm{dl}$ $(6.1 \mathrm{mmol} / \mathrm{l})$ in surgical patients in critical care units (328-330). However, in several studies of critically ill medical patients $(330,331,333)$, the incidence of severe hypoglycemia (blood glucose $<40$ $\mathrm{mg} / \mathrm{dl}$ ) was approximately threefold greater in intensively treated patients. The identification of hypoglycemia as an independent risk factor for death in the medical ICU population (334) may merit caution in widely promoting the 80-110 $\mathrm{mg} / \mathrm{dl}$ target range for all critically ill populations.

For patients in general medicalsurgical units, the evidence for specific glycemic goals is less definitive. Epidemiologic and physiologic data suggest that higher blood glucose levels are associated with worse outcomes, but whether glucose is simply a marker of the severity of underlying illness or a mediator of adverse outcomes is unclear. Glycemic targets similar to those of outpatients may be difficult to achieve in the hospital due to the effects of stress hyperglycemia, altered nutritional intake, and multiple interruptions to medical care. Blood glucose levels shown to be associated with improved outcomes in these patients (fasting glucose $<126 \mathrm{mg} / \mathrm{dl}$ and all blood glucose readings $<180-200 \mathrm{mg} / \mathrm{dl}$ ) would appear reasonable, if they can be safely achieved.

In both the critical care and noncritical care venue, glycemic goals must take into account the individual patient's situation as well as hospital system support for achieving these goals. A continuous quality improvement strategy may facilitate gradual improvement in mean glycemia hospital wide.

\section{Treatment options in hospitalized patients}

a. Noninsulin glucose-lowering agents. No large studies have investigated the potential roles of various noninsulin glucose-lowering agents on outcomes of hospitalized patients with diabetes. Use of the various noninsulin classes in the inpatient setting presents some specific issues.

The long action of sulfonylureas and their predisposition to hypoglycemia in patients not consuming their normal nutrition serve as relative contraindications to routine use of these agents in the hospital (335). While the meglitinides, repa- 
glinide and neteglinide, theoretically would produce less hypoglycemia than sulfonylureas, lack of clinical trial data for these agents, and the fact that they are primarily prandial in effect, would preclude their use. The major limitation to metformin use in the hospital is a number of specific contraindications to its use, related to risk of lactic acidosis, many of which occur in the hospital. The most common risk factors for lactic acidosis in metformin-treated patients are cardiac disease, including decompensated CHF, hypoperfusion, renal insufficiency, old age, and chronic pulmonary disease (336). Lactic acidosis is a rare complication in the outpatient setting (337), despite the relative frequency of risk factors (338). However, in the hospital the risks of hypoxia, hypoperfusion, and renal insufficiency are much higher, and it is prudent to avoid the use of metformin in most patients.

TZDs are not suitable for initiation in the hospital because of their delayed onset of effect. In addition, they increase intravascular volume, a particular problem in those predisposed to CHF and potentially a problem for patients with hemodynamic changes related to admission diagnoses (e.g., acute coronary ischemia) or interventions common in hospitalized patients. Pramlintide and exenatide work mainly by reducing postprandial hyperglycemia and would therefore not be appropriate for patients not eating (nil per os, NPO) or with reduced caloric consumption. Furthermore, initiation of these drugs in the inpatient setting would be problematic due to alterations in normal food intake and their propensity to induce nausea initially. There is limited experience, and no published data, on the DPP-IV inhibitors in the hospital setting, although there are no specific safety concerns. They are mainly effective on postprandial glucose and therefore would have limited effect in patients who are not eating.

In summary, each of the major classes of noninsulin glucose-lowering drugs has significant limitations for inpatient use. Additionally, they provide little flexibility or opportunity for titration in a setting where acute changes often demand these characteristics. Therefore, insulin, when used properly, is preferred for the majority of hyperglycemic patients in the hospital setting.

\section{b. Insulin}

i. Subcutaneous insulin therapy. Subcutaneous insulin therapy may be used to at- tain glucose control in most hospitalized patients with diabetes outside of the critical care arena. The components of the daily insulin dose requirement can be met by a variety of insulins, depending on the particular hospital situation. Subcutaneous insulin therapy should cover both basal and nutritional needs and is subdivided into scheduled insulin and supplemental, or correction-dose, insulin. Correction-dose insulin therapy is an important adjunct to scheduled insulin, both as a dose-finding strategy and as a supplement when rapid changes in insulin requirements lead to hyperglycemia. If correction doses are frequently required, the appropriate scheduled insulin doses should be increased to accommodate the increased insulin needs. There are currently no published studies comparing human regular insulin with rapid-acting analogs for use as correction-dose insulin.

The traditional "sliding-scale" insulin regimens, usually consisting of regular insulin without any intermediate or longacting insulins, have been shown to be ineffective when used as monotherapy in patients with an established insulin requirement (339-341). Problems with sliding-scale insulin regimens include the fact that the sliding-scale regimen prescribed on admission is likely to be used throughout the hospital stay without modification, even when control remains poor. Additionally, sliding-scale insulin therapy treats hyperglycemia after it has already occurred, instead of preventing the occurrence of hyperglycemia. This "reactive" approach can lead to rapid changes in blood glucose levels, which may exacerbate both hyper- and hypoglycemia.

A recent study demonstrated the safety and efficacy of using basal-bolus insulin therapy utilizing weight-based dosing in insulin-naïve hospitalized patients with type 2 diabetes (342). Glycemic control, defined as a mean blood glucose $<140 \mathrm{mg} / \mathrm{dl}$, was achieved in $68 \%$ of patients receiving basal-bolus insulin versus only $38 \%$ of those receiving sliding-scale insulin alone. There were no differences in hypoglycemia between the two groups. It is important to note that the patients in this study were obese, and the doses used in this study ( 0.4 to 0.5 units $\cdot \mathrm{kg} \cdot$ day $^{-1}$ ) are higher than what may be required in patients who are more sensitive to insulin, such as those who are lean or who have type 1 diabetes.

ii. Intravenous insulin infusion. The only method of insulin delivery specifically de- veloped for use in the hospital is continuous intravenous infusion, using regular crystalline insulin. There is no advantage to using rapid acting analogs, whose structural modifications increase the rate of absorption from subcutaneous depots, in an intravenous insulin infusion. The medical literature supports the use of intravenous insulin infusion in preference to the subcutaneous route of insulin administration for several clinical indications among nonpregnant adults. These include DKA and nonketotic hyperosmolar state; general preoperative, intraoperative, and postoperative care; the postoperative period following heart surgery; following organ transplantation; with cardiogenic shock; exacerbated hyperglycemia during high-dose glucocorticoid therapy; type 1 patients who are NPO; or in critical care illness in general. It may be used as a dose-finding strategy in anticipation of initiation or reinitiation of subcutaneous insulin therapy in type 1 or type 2 diabetes.

Many institutions use insulin infusion algorithms that can be implemented by nursing staff. Although numerous algorithms have been published, there have been no head-to-head comparisons between insulin infusion strategies. Algorithms should incorporate the concepts that maintenance requirements differ between patients and change over the course of treatment. Ideally, intravenous insulin algorithms should consider both current and previous glucose levels, the rate of change of plasma glucose, and the current intravenous insulin infusion rate. For all algorithms, frequent (Q $1-2 \mathrm{~h}$ ) bedside glucose testing is required.

iii. Transition from intravenous to subcutaneous insulin therapy. For those who will require subcutaneous insulin, the very short half-life of intravenous insulin necessitates administering the first dose of subcutaneous insulin before discontinuation of the intravenous insulin infusion. If short or rapid-acting insulin is used, it should be injected $1-2 \mathrm{~h}$ before stopping the infusion. If intermediate- or longacting insulin is used alone, it should be injected 2-3 h before. A combination of short/rapid- and intermediate/longacting insulin is usually preferred. Basal insulin therapy can be initiated at any time of the day and should not be withheld to await a specific dosing time, such as bedtime. A recent clinical trial demonstrated that a regimen using $80 \%$ of the intravenous insulin requirement over the preceding $24 \mathrm{~h}$, divided into basal and 
bolus insulin components, was effective at achieving blood glucose levels between 80 and $150 \mathrm{mg} / \mathrm{dl}$ following discontinuation of the intravenous insulin (343).

\section{Self-management in the hospital} Self-management of diabetes in the hospital may be appropriate for competent adult patients who have a stable level of consciousness, have reasonably stable daily insulin requirements, successfully conduct self-management of diabetes at home, have physical skills needed to successfully self-administer insulin and perform SMBG, have adequate oral intake, and are proficient in carbohydrate counting, use of multiple daily insulin injections or insulin pump therapy, and sickday management. The patient and physician, in consultation with nursing staff, must agree that patient selfmanagement is appropriate under the conditions of hospitalization. For patients conducting self-management in the hospital, it is imperative that basal, prandial, and correction doses of insulin and results of bedside glucose monitoring be recorded as part of the patient's hospital medical record. While many institutions allow patients on insulin pumps to continue these devices in the hospital, others express concern regarding use of a device unfamiliar to staff, particularly in patients who are not able to manage their own pump therapy. If a patient is too ill to self-manage either multiple daily injections or CSII, then appropriate subcutaneous doses can be calculated on the basis of their basal and bolus insulin needs during hospitalization, with adjustments for changes in nutritional or metabolic status.

\section{Preventing hypoglycemia}

Hypoglycemia, especially in insulintreated patients, is the leading limiting factor in the glycemic management of type 1 and type 2 diabetes (146). In the hospital, multiple additional risk factors for hypoglycemia are present, even among patients who are neither "brittle" nor tightly controlled. Patients with or without diabetes may experience hypoglycemia in the hospital in association with altered nutritional state, heart failure, renal or liver disease, malignancy, infection, or sepsis $(344,345)$. Additional triggering events leading to iatrogenic hypoglycemia include sudden reduction of corticosteroid dose, altered ability of the patient to self-report symptoms, reduction of oral intake, emesis, new NPO status, inappropriate timing of short- or rapid-acting insulin in relation to meals, reduction of rate of administration of intravenous dextrose, and unexpected interruption of enteral feedings or parenteral nutrition.

Despite the preventable nature of many inpatient episodes of hypoglycemia, institutions are more likely to have nursing protocols for the treatment of hypoglycemia than for its prevention. Tracking such episodes and analyzing their causes are important quality improvement activities.

\section{Diabetes care providers in the hospital}

Inpatient diabetes management may be effectively provided by primary care physicians, endocrinologists, or hospitalists, but involvement of appropriately trained specialists or specialty teams may reduce length of stay, improve glycemic control, and improve outcomes (346-349). In the care of diabetes, implementation of standardized order sets for scheduled and correction-dose insulin may reduce reliance on sliding-scale management. A team approach is needed to establish hospital pathways. To achieve glycemic targets associated with improved hospital outcomes, hospitals will need multidisciplinary support for using insulin infusion therapy outside of critical care units or will need to develop protocols for subcutaneous insulin therapy that effectively and safely achieve glycemic targets (350).

\section{DSME in the hospital}

Teaching diabetes self-management to patients in hospitals is a challenging task. Patients are ill, under increased stress related to their hospitalization and diagnosis, and in an environment not conducive to learning. Ideally, people with diabetes should be taught at a time and place conducive to learning, as outpatients in a recognized program of diabetes education.

For the hospitalized patient, diabetes "survival skills" education is generally a feasible approach. Patients and/or family members receive sufficient information and training to enable safe care at home. Those newly diagnosed with diabetes or who are new to insulin and/or blood glucose monitoring need to be instructed before discharge. Those patients hospitalized because of a crisis related to diabetes management or poor care at home need education to prevent subsequent episodes of hospitalization. An assessment of the need for a home health referral or referral to an outpatient diabetes education pro- gram should be part of discharge planning for all patients.

\section{MNT in the hospital}

Hospital diets continue to be ordered by calorie levels based on the "ADA diet." However, since 1994 the ADA has not endorsed any single meal plan or specified percentages of macronutrients, and the term "ADA diet" should no longer be used. Current nutrition recommendations advise individualization based on treatment goals, physiologic parameters, and medication usage. Because of the complexity of nutrition issues in the hospital, a registered dietitian, knowledgeable and skilled in MNT, should serve as an inpatient team member. The dietitian is responsible for integrating information about the patient's clinical condition, eating, and lifestyle habits and for establishing treatment goals in order to determine a realistic plan for nutrition therapy $(351,352)$.

\section{Bedside blood glucose monitoring} Implementing intensive diabetes therapy in the hospital setting requires frequent and accurate blood glucose data. This measure is analogous to an additional "vital sign" for hospitalized patients with diabetes. Bedside glucose monitoring using capillary blood has advantages over laboratory venous glucose testing because the results can be obtained rapidly at the "point of care," where therapeutic decisions are made.

Bedside blood glucose testing is usually performed with portable meters that are similar or identical to devices for home SMBG. Staff training and ongoing quality control activities are important components of ensuring accuracy of the results. Ability to track the occurrence of hypo- and hyperglycemia is necessary. Results of bedside glucose tests should be readily available to all members of the care team.

For patients who are eating, commonly recommended testing frequencies are premeal and at bedtime. For patients not eating, testing every $4-6 \mathrm{~h}$ is usually sufficient for determining correction insulin doses. Patients on continuous intravenous insulin typically require hourly blood glucose testing until the blood glucose levels are stable, then every $2 \mathrm{~h}$.

\section{Discharge planning}

It is important to anticipate the postdischarge antihyperglycemic regimen in all patients with diabetes or newly discov- 
ered hyperglycemia. The optimal program will need to consider the type and severity of diabetes, the effects of the patient's illness on blood glucose levels, and the capacities and desires of the patient. Smooth transition to outpatient care should be ensured, especially in those new to insulin therapy or in whom the diabetes regimen has been substantially altered during the hospitalization. All patients in whom the diagnosis of diabetes is new should have, at minimum, "survival skills" training before discharge. More expanded diabetes education can be arranged in the community. For those with hyperglycemia who do not require treatment upon discharge, follow-up testing through their primary care physician should be arranged, since many of these individuals are found to have diabetes when tested after discharge.

\section{B. Diabetes care in the school and day care setting (353)}

\section{Recommendations}

- An individualized Diabetes Medical Management Plan (DMMP) should be developed by the parent/guardian and the student's personal diabetes health care team with input from the parent/ guardian. (E)

- All school staff members who have responsibility for a student with diabetes should receive training that provides a basic understanding of diabetes and a student's needs. (E)

- While the school nurse is the coordinator and primary provider of diabetes care, a small number of school personnel should be trained in routine and emergency diabetes procedures (including monitoring of blood glucose levels and administration of insulin and glucagon) and in the appropriate response to high and low blood glucose levels and should perform these diabetes care tasks when the school nurse is not available to do so. These school personnel need not be health care professionals. (E)

- As specified in the DMMP and as developmentally appropriate, the student with diabetes should have immediate access to diabetes supplies at all times and should be permitted to self-manage his or her diabetes in the classroom or anywhere the student may be in conjunction with a school activity. Such self-management should include blood glucose monitoring and responding to blood glucose levels with needed food and medication. (E)

There are $\sim 186,300$ individuals $<20$ years of age with diabetes in the U.S., most of whom attend school and/or some type of day care and need knowledgeable staff to provide a safe environment. Despite legal protections, including coverage of children with diabetes under Section 504 of the Rehabilitation Act of 1973, the Individuals with Disabilities Education Act, and the Americans with Disabilities Act, children in the school and day care setting still face discrimination. The ADA position statement on Diabetes Care in the School and Day Care Setting (353) provides the legal and medical justifications for the recommendations provided herein.

Appropriate diabetes care in the school and day care setting is necessary for the child's immediate safety, longterm well-being, and optimal academic performance. Parents and the health care team should provide school systems and day care providers with the information necessary for children with diabetes to participate fully and safely in the school/ day care experience by developing an individualized DMMP.

The school nurse should be the key coordinator and provider of care and should coordinate the training of an adequate number of school personnel and ensure that if the school nurse is not present at least one adult is present who is trained to perform the necessary diabetes procedures (e.g., blood glucose monitoring and insulin and glucagon administration) and provide the appropriate response to high and low blood glucose levels in a timely manner while the student is at school, on field trips, participating in school-sponsored extracurricular activities, and on transportation provided by the school or day care facility. These school personnel need not be health care professionals.

The student with diabetes should have immediate access to diabetes supplies at all times, with supervision as needed. The student should be able to obtain a blood glucose level and respond to the results as quickly and conveniently as possible in the classroom or wherever the child is in conjunction with a school-related activity, minimizing the need for missing instruction in the classroom and avoiding the risk of worsening hypoglycemia or hyperglycemia if the child must go somewhere else for treatment. The student's desire for privacy dur- ing blood glucose monitoring and insulin administration should also be accommodated.

The ADA and partner organizations have developed tools for school personnel to provide a safe and nondiscriminatory educational environment for all students with diabetes $(354,355)$.

\section{Diabetes care at diabetes camps (356)}

\section{Recommendations}

- Each camper should have a standardized medical form completed by his/her family and the physician managing the diabetes. (E)

- Camp medical staff should be led by with a physician with expertise in managing type 1 and type 2 diabetes and include nurses (including diabetes educators and diabetes clinical nurse specialists) and registered dietitians with expertise in diabetes. (E)

- All camp staff, including physicians, nurses, dietitians, and volunteers, should undergo background testing to ensure appropriateness in working with children. (E)

The concept of specialized residential and day camps for children with diabetes has become widespread throughout the U.S. and many other parts of the world. The mission of diabetes camps is to provide a camping experience in a safe environment. An equally important goal is to enable children with diabetes to meet and share their experiences with one another while they learn to be more personally responsible for their disease. For this to occur, a skilled medical and camping staff must be available to ensure optimal safety and an integrated camping/educational experience.

Each camper should have a standardized medical form completed by his/her family and the physician managing the diabetes that details the camper's past medical history, immunization record, and diabetes regimen. The home insulin dosage should be recorded for each camper, including type(s) of insulin used, number and timing of injections, and the correction factor and carbohydrate ratios used for determining bolus dosages for basalbolus regimens. Campers using CSII should also have their basal rates specified. Because camp is often associated with more physical activity than experienced at home, the insulin dose may have to be decreased during camp. 
The diabetes camping experience is short term, with food and activity different than the home environment. Thus, goals of glycemic control at camp are to avoid extremes in blood glucose levels rather than attempting optimization of intensive glycemic control.

During camp, a daily record of the camper's progress should be made, including all blood glucose levels and insulin dosages. To ensure safety and optimal diabetes management, multiple blood glucose determinations should be made throughout each 24-h period: before meals, at bedtime, after or during prolonged and strenuous activity, and in the middle of the night when indicated for prior hypoglycemia. If major alterations of a camper's regimen appear to be indicated, it is important to discuss this with the camper and the family in addition to the child's local physician. The record of what transpired during camp should be discussed with the family at the end of the camp session and a copy sent to the child's physician.

Each camp should secure a formal relationship with a nearby medical facility so that camp medical staff can refer to this facility for prompt treatment of medical emergencies. ADA requires that the camp medical director be a physician with expertise in managing type 1 and type 2 diabetes. Nursing staff should include diabetes educators and diabetes clinical nurse specialists. Registered dietitians with expertise in diabetes should have input into the design of the menu and the education program. All camp staff, including medical, nursing, nutrition, and volunteer, should undergo background testing to ensure appropriateness in working with children.

\section{Diabetes management in correctional institutions (357)}

\section{Recommendations}

- Correctional staff should be trained in the recognition, treatment, and appropriate referral for hypo- and hyperglycemia, including serious metabolic decompensation. (E)

- Patients with a diagnosis of diabetes should have a complete medical history and physical examination by a licensed health care provider with prescriptive authority in a timely manner upon entry. Insulin-treated patients should have a capillary blood glucose (CBG) determination within $1-2 \mathrm{~h}$ of arrival. Staff should identify patients with type
1 diabetes who are at high risk for DKA with omission of insulin. (E)

- Medications and MNT should be continued without interruption upon entry into the correctional environment. (E)

- In the correctional setting, policies and procedures should enable CBG monitoring to occur at the frequency necessitated by the patient's glycemic control and diabetes regimen and should require staff to notify a physician of all CBG results outside of a specified range, as determined by the treating physician. (E)

- For all inter-institutional transfers, a medical transfer summary should be transferred with the patient, and diabetes supplies and medication should accompany the patient. (E)

- Correctional staff should begin discharge planning with adequate lead time to ensure continuity of care and facilitate entry into community diabetes care. (E)

At any given time, over 2 million people are incarcerated in prisons and jails in the U.S., and it is estimated that nearly 80,000 of these inmates have diabetes. In addition, many more people with diabetes pass through the corrections system in a given year.

People with diabetes in correctional facilities should receive care that meets national standards. Correctional institutions have unique circumstances that need to be considered so that all standards of care may be achieved. Correctional institutions should have written policies and procedures for the management of diabetes and for training of medical and correctional staff in diabetes care practices.

Reception screening should emphasize patient safety. In particular, rapid identification of all insulin-treated individuals with diabetes is essential in order to identify those at highest risk for hypoand hyperglycemia and DKA. All insulintreated patients should have a CBG determination within $1-2 \mathrm{~h}$ of arrival. Patients with a diagnosis of diabetes should have a complete medical history and physical examination by a licensed health care provider with prescriptive authority in a timely manner. It is essential that medication and MNT be continued without interruption upon entry into the correctional system, as a hiatus in either medication or appropriate nutrition may lead to either severe hyper- or hypoglycemia.

Patients must have access to prompt treatment of hypo- and hyperglycemia. Correctional staff should be trained in the recognition and treatment of these conditions, and appropriate staff should be trained to administer glucagon. Institutions should implement a policy requiring staff to notify a physician of all CBG results outside of a specified range, as determined by the treating physician.

Correctional institutions should have systems in place to ensure that insulin administration and meals are coordinated to prevent hypo- and hyperglycemia, taking into consideration the transport of residents off site and the possibility of emergency schedule changes. The frequency of CBG monitoring will vary by patients' glycemic control and diabetes regimens. Policies and procedures should ensure that the health care staff has adequate knowledge and skills to direct the management and education of individuals with diabetes.

Patients in jails may be housed for a short period of time before being transferred or released, and patients in prison may be transferred within the system several times during their incarceration. Transferring a patient with diabetes from one correctional facility to another requires a coordinated effort, as does planning for discharge. The ADA position statement on Diabetes Management in Correctional Institutions (357) should be consulted for more information on this topic.

\section{E. Emergency and disaster preparedness (358)}

\section{Recommendations}

- People with diabetes should maintain a disaster kit that includes items important to their diabetes self-management and continuing medical care. (E)

- The kit should be reviewed and replenished at least twice yearly. (E)

The difficulties encountered by people with diabetes and their health care providers in the wake of Hurricane Katrina (359) highlight the need for people with diabetes to be prepared for emergencies, whether natural or otherwise, affecting a region or just their household. Such preparedness will lessen the impact an emergency may have on their condition. It is recommended that people with diabetes keep a waterproof and insulated disaster kit ready with items critically important to their self-management. These may include glucose testing strips, lancets, and a glucose-testing meter; medications in- 
cluding insulin in a cool bag; syringes; glucose tabs or gels; antibiotic ointments/ creams for external use; glucagon emergency kits; and photocopies of relevant medical information, particularly medication lists and recent lab tests/ procedures if available. If possible, prescription numbers should be noted, since many chain pharmacies throughout the country will refill medications based on the prescription number alone. In addition, it may be important to carry a list of contacts for national organizations, such as the American Red Cross and ADA. This disaster kit should be reviewed and replenished at least twice yearly.

\section{DIABETES AND EMPLOYMENT (360)}

\section{Recommendations}

- When questions arise about the medical fitness of a person with diabetes for a particular job, a health care professional with expertise in treating diabetes should perform an individualized assessment; input from the treating physician should always be included. (E)

- Proper safety assessments for employment should include review of blood glucose test results, history of severe hypoglycemia, presence of hypoglycemia unawareness, and presence of diabetes-related complications but should not include urine glucose or AlC/eAG tests or be based on a general assessment of level of control. (E)

Any person with diabetes, whether insulin treated or noninsulin treated, should be eligible for any employment for which he/she is otherwise qualified. Questions are sometimes raised by employers about the safety and effectiveness of individuals with diabetes in a given job. When such questions are legitimately raised, a person with diabetes should be individually assessed by a health care professional with expertise in diabetes to determine whether or not that person can safely and effectively perform the particular duties of the job in question.

Employment decisions should never be based on generalizations or stereotypes regarding the effects of diabetes. "Blanket bans" that restrict individuals with diabetes from certain jobs or classes of employment solely because of the diagnosis of diabetes or the use of insulin are medically and legally inappropriate and ignore the many advancements in diabetes management that range from the types of medications used to the tools used to administer them and to monitor blood glucose levels. For most types of employment, there is no reason to believe that the individual's diabetes will put employees or the public at risk. In certain safety-sensitive positions the safety concern is whether the employee will become suddenly disoriented or incapacitated. Episodes of severe hypoglycemia should be examined by a health care professional with expertise in diabetes to determine any impact on safe performance of the job. Hyperglycemia is not typically a barrier to employment unless long-term complications are present that interfere with the performance of the job.

Most accommodations that help an individual with diabetes do his or her job may be provided easily and with little or no cost to the employer. Typical accommodations include breaks to test blood glucose, administer insulin, or access food and beverages. Some individuals may need leave or a flexible work schedule or accommodations for diabetesrelated complications.

The ADA position statement on Diabetes and Employment should be consulted for more information on this topic.

\section{THIRD-PARTY REIMBURSEMENT FOR DIABETES CARE, SELF. MANAGEMENT EDUCATION, AND SUPPLIES (361)}

\section{Recommendations}

- Patients and practitioners should have access to all classes of antidiabetic medications, equipment, and supplies without undue controls. (E)

- MNT and DSME should be covered by insurance and other payors. (E)

To achieve optimal glucose control, the person with diabetes must be able to access health care providers who have expertise in the field of diabetes. Treatments and therapies that improve glycemic control and reduce the complications of diabetes will also significantly reduce health care costs. Access to the integral components of diabetes care, such as health care visits, diabetes supplies and medications, and self-management education, is essential. All medications and supplies, such as syringes, strips, and meters, related to the daily care of diabetes must also be reimbursed by third-party payors.

It is recognized that the use of formu- laries, prior authorization, and provisions such as competitive bidding can manage provider practices as well as costs to the potential benefit of payors and patients. However, any controls should ensure that all classes of anti-diabetic agents with unique mechanisms of action and all classes of equipment and supplies designed for use with such equipment are available to facilitate achieving glycemic goals and to reduce the risk of complications. Without appropriate safeguards, undue controls could constitute an obstruction of effective care.

Medicare and many other third-party payors cover DSME (Centers for Medicare and Medicaid Services [CMS] term is diabetes self-management training [DSMT]) that meets the national standards for DSME (107) and MNT. The qualified beneficiary, with referral from the provider managing his or her diabetes, can receive an initial benefit of $10 \mathrm{~h}$ of DSMT and $3 \mathrm{~h}$ of MNT, with a potential total of $13 \mathrm{~h}$ of initial. More information on Medicare policy, including follow-up benefits, is available at www.diabetes.org/forhealth-professionals-and-scientists/ recognition.jsp or on the CMS Web sites: DSME, www.cms.hhs.gov/DiabetesSelfManagement; and diabetes MNT, www. cms.hhs.gov/MedicalNutritionTherapy, reimbursement.

\section{STRATEGIES FOR IMPROVING DIABETES CARE}

The implementation of the standards of care for diabetes has been suboptimal in most clinical settings. A recent report (362) indicated that only $37 \%$ of adults with diagnosed diabetes achieved an AlC of $<7 \%$, only $36 \%$ had a blood pressure $<130 / 80 \mathrm{mmHg}$, and just $48 \%$ had a total cholesterol $<200 \mathrm{mg} / \mathrm{dl}$. Most distressing was that only $7.3 \%$ of people with diabetes achieved all three treatment goals.

While numerous interventions to improve adherence to the recommended standards have been implemented, the challenge of providing uniformly effective diabetes care has thus far defied a simple solution. A major contributor to suboptimal care is a delivery system that too often is fragmented, lacks clinical information capabilities, often duplicates services, and is poorly designed for the delivery of chronic care. The Institute of Medicine has called for changes so that delivery systems provide care that is evidence based, patient centered, and systems oriented and takes advantage of information tech- 
nologies that foster continuous quality improvement. Collaborative, multidisciplinary teams should be best suited to provide such care for people with chronic conditions like diabetes and to empower patients' performance of appropriate selfmanagement. Alterations in reimbursement that reward the provision of quality care, as defined by the attainment of quality measures developed by such programs as the ADA/National Committee for Quality Assurance Diabetes Provider Recognition Program will also be required to achieve desired outcome goals.

The NDEP recently launched a new online resource to help health care professionals better organize their diabetes care. The www.betterdiabetescare.nih.gov Web site should help users design and implement more effective health care delivery systems for those with diabetes.

In recent years, numerous health care organizations, ranging from large health care systems such as the U.S. Veterans Administration to small private practices, have implemented strategies to improve diabetes care. Successful programs have published results showing improvement in process measures such as measurement of AlC, lipids, and blood pressure. Effects on important intermediate outcomes, such as mean AlC for populations, have been more difficult to demonstrate (363365), although examples do exist (366370). Successful interventions have been focused at the level of health care professionals, delivery systems, and patients. Features of successful programs reported in the literature include:

- Improving health care professional education regarding the standards of care through formal and informal education programs.

- Delivery of DSME, which has been shown to increase adherence to standard of care.

- Adoption of practice guidelines, with participation of health care professionals in the process. Guidelines should be readily accessible at the point of service, such as on patient charts, in examining rooms, in "wallet or pocket cards," on PDAs, or on office computer systems. Guidelines should begin with a summary of their major recommendations instructing health care professionals what to do and how to do it.

- Use of checklists that mirror guidelines have been successful at improving adherence to standards of care.

- Systems changes, such as provision of automated reminders to health care professionals and patients, reporting of process and outcome data to providers, and especially identification of patients at risk because of failure to achieve target values or a lack of reported values.

- Quality improvement programs combining continuous quality improvement or other cycles of analysis and intervention with provider performance data.

- Practice changes, such as clustering of dedicated diabetes visits into specific times within a primary care practice schedule and/or visits with multiple health care professionals on a single day and group visits.

- Tracking systems with either an electronic medical record or patient registry have been helpful at increasing adherence to standards of care by prospectively identifying those requiring assessments and/or treatment modifications. They likely could have greater efficacy if they suggested specific therapeutic interventions to be considered for a particular patient at a particular point in time (371).

- A variety of nonautomated systems, such as mailing reminders to patients, chart stickers, and flow sheets, have been useful to prompt both providers and patients.

- Availability of case or (preferably) care management services, usually by a nurse (372). Nurses, pharmacists, and other nonphysician health care professionals using detailed algorithms working under the supervision of physicians and/or nurse education calls have also been helpful. Similarly, dietitians using MNT guidelines have been demonstrated to improve glycemic control.

- Availability and involvement of expert consultants, such as endocrinologists and diabetes educators.

Evidence suggests that these individual initiatives work best when provided as components of a multifactorial intervention. Therefore, it is difficult to assess the contribution of each component; however, it is clear that optimal diabetes management requires an organized, systematic approach and involvement of a coordinated team of health care professionals.

\section{References}

1. American Diabetes Association: Medical Management of Type 1 Diabetes. 5th ed.
Alexandria, VA, American Diabetes Association, 2008

2. American Diabetes Association: Medical Management of Type 2 Diabetes. 6th ed. Alexandria, VA, American Diabetes Association, 2008

3. American Diabetes Association: Intensive Diabetes Management. Alexandria, VA, American Diabetes Association, 2003

4. Expert Committee on the Diagnosis and Classification of Diabetes Mellitus: Report of the Expert Committee on the Diagnosis and Classification of Diabetes Mellitus. Diabetes Care 20:1183-1197, 1997

5. Expert Committee on the Diagnosis and Classification of Diabetes Mellitus: Follow-up report on the diagnosis of diabetes mellitus. Diabetes Care 26:31603167,2003

6. Davidson MB, Schriger DL, Peters AL, Lorber B: Relationship between fasting plasma glucose and glycosylated hemoglobin: potential for false-positive diagnoses of type 2 diabetes using new diagnostic criteria. JAMA 281:12031210, 1999

7. Nathan DM, Davidson MB, DeFronzo RA, Heine RJ, Henry RR, Pratley R, Zinman B: Impaired fasting glucose and impaired glucose tolerance: implications for care. Diabetes Care 30:753-759, 2007

8. Engelgau MM, Narayan KM, Herman WH: Screening for type 2 diabetes. Diabetes Care 23:1563-1580, 2000

9. Gabir MM, Hanson RL, Dabelea D, Imperatore G, Roumain J, Bennett PH, Knowler WC: The 1997 American Diabetes Association and 1999 World Health Organization criteria for hyperglycemia in the diagnosis and prediction of diabetes. Diabetes Care 23:11081112, 2000

10. Knowler WC, Barrett-Connor E, Fowler SE, Hamman RF, Lachin JM, Walker EA, Nathan DM: Reduction in the incidence of type 2 diabetes with lifestyle intervention or metformin. $N$ Engl J Med 346: 393-403, 2002

11. Tuomilehto J, Lindstrom J, Eriksson JG, Valle TT, Hamalainen H, Ilanne-Parikka P, Keinanen-Kiukaanniemi S, Laakso M, Louheranta A, Rastas M, Salminen V, Uusitupa M: Prevention of type 2 diabetes mellitus by changes in lifestyle among subjects with impaired glucose tolerance. N Engl J Med 344:1343-1350, 2001

12. Pan XR, Li GW, Hu YH, Wang JX, Yang WY, An ZX, Hu ZX, Lin J, Xiao JZ, Cao HB, Liu PA, Jiang XG, Jiang YY, Wang JP, Zheng H, Zhang H, Bennett PH, Howard BV: Effects of diet and exercise in preventing NIDDM in people with impaired glucose tolerance: the Da Qing IGT and Diabetes Study. Diabetes Care 20:537544,1997 
13. Buchanan TA, Xiang AH, Peters RK, Kjos SL, Marroquin A, Goico J, Ochoa C, Tan S, Berkowitz K, Hodis HN, Azen SP: Preservation of pancreatic $\beta$-cell function and prevention of type 2 diabetes by pharmacological treatment of insulin resistance in high-risk hispanic women. Diabetes 51:2796-2803, 2002

14. Chiasson JL, Josse RG, Gomis R, Hanefeld M, Karasik A, Laakso M: Acarbose for prevention of type 2 diabetes mellitus: the STOP-NIDDM randomised trial. Lancet 359:2072-2077, 2002

15. Gerstein HC, Yusuf S, Bosch J, Pogue J, Sheridan P, Dinccag N, Hanefeld M, Hoogwerf B, Laakso M, Mohan V, Shaw J, Zinman B, Holman RR: Effect of rosiglitazone on the frequency of diabetes in patients with impaired glucose tolerance or impaired fasting glucose: a randomised controlled trial. Lancet 368:10961105,2006

16. Ramachandran A, Snehalatha C, Mary S, Mukesh B, Bhaskar AD, Vijay V: The Indian Diabetes Prevention Programme shows that lifestyle modification and metformin prevent type 2 diabetes in Asian Indian subjects with impaired glucose tolerance (IDPP-1). Diabetologia 49: 289-297, 2006

17. Johnson SL, Tabaei BP, Herman WH: The efficacy and cost of alternative strategies for systematic screening for type 2 diabetes in the U.S. population 45-74 years of age. Diabetes Care 28:307-311, 2005

18. Harris R, Donahue K, Rathore SS, Frame P, Woolf SH, Lohr KN: Screening adults for type 2 diabetes: a review of the evidence for the U.S. Preventive Services Task Force. Ann Intern Med 138:215229, 2003

19. USPSTF: Screening for type 2 diabetes mellitus in adults: recommendations and rationale. Ann Intern Med 138:212214, 2003

20. Dabelea D, Bell RA, D'Agostino RB Jr, Imperatore G, Johansen JM, Linder B, Liu LL, Loots B, Marcovina S, MayerDavis EJ, Pettitt DJ, Waitzfelder B: Incidence of diabetes in youth in the United States. JAMA 297:2716-2724, 2007

21. Liese AD, D'Agostino RB Jr, Hamman RF, Kilgo PD, Lawrence JM, Liu LL, Loots B, Linder B, Marcovina S, Rodriguez B, Standiford D, Williams DE: The burden of diabetes mellitus among US youth: prevalence estimates from the SEARCH for Diabetes in Youth Study. Pediatrics 118:1510-1518, 2006

22. American Diabetes Association: Type 2 diabetes in children and adolescents (Consensus Statement). Diabetes Care 23:381-389, 2000

23. American Diabetes Association: Gestational diabetes mellitus (Position Statement). Diabetes Care 27 (Suppl. 1):S88S90, 2004
24. Metzger BE, Lowe LP, Dyer AR, Trimble ER, Chaovarindr U, Coustan DR, Hadden DR, McCance DR, Hod M, McIntyre HD, Oats JJ, Persson B, Rogers MS, Sacks DA: Hyperglycemia and adverse pregnancy outcomes. N Engl J Med 358: 1991-2002, 2008

25. Kim C, Newton KM, Knopp RH: Gestational diabetes and the incidence of type 2 diabetes: a systematic review. Diabetes Care 25:1862-1868, 2002

26. Lindstrom J, Ilanne-Parikka P, Peltonen M, Aunola S, Eriksson JG, Hemio K, Hamalainen $\mathrm{H}$, Harkonen $\mathrm{P}$, KeinanenKiukaanniemi S, Laakso M, Louheranta A, Mannelin M, Paturi M, Sundvall J, Valle TT, Uusitupa M, Tuomilehto J: Sustained reduction in the incidence of type 2 diabetes by lifestyle intervention: follow-up of the Finnish Diabetes Prevention Study. Lancet 368:1673-1679, 2006

27. Li G, Zhang P, Wang J, Gregg EW, Yang W, Gong Q, Li H, Li H, Jiang Y, An Y, Shuai Y, Zhang B, Zhang J, Thompson TJ, Gerzoff RB, Roglic G, Hu Y, Bennett PH: The long-term effect of lifestyle interventions to prevent diabetes in the China Da Qing Diabetes Prevention Study: a 20-year follow-up study. Lancet 371:1783-1789, 2008

28. Kosaka K, Noda M, Kuzuya T: Prevention of type 2 diabetes by lifestyle intervention: a Japanese trial in IGT males. Diabetes Res Clin Pract 67:152-162, 2005

29. Torgerson JS, Hauptman J, Boldrin MN, Sjostrom L: XENical in the prevention of diabetes in obese subjects (XENDOS) study: a randomized study of orlistat as an adjunct to lifestyle changes for the prevention of type 2 diabetes in obese patients. Diabetes Care 27:155-161, 2004

30. Gerstein HC: Point: If it is important to prevent type 2 diabetes, it is important to consider all proven therapies within a comprehensive approach. Diabetes Care 30:432-434, 2007

31. American Diabetes Association: Consensus statement on self-monitoring of blood glucose. Diabetes Care 10:95-99, 1987

32. American Diabetes Asociation: Selfmonitoring of blood glucose. Diabetes Care 17:81-86, 1994

33. Welschen LM, Bloemendal E, Nijpels G, Dekker JM, Heine RJ, Stalman WA, Bouter LM: Self-monitoring of blood glucose in patients with type 2 diabetes who are not using insulin: a systematic review. Diabetes Care 28:1510-1517, 2005

34. Farmer A, Wade A, Goyder E, Yudkin P, French D, Craven A, Holman R, Kinmonth AL, Neil A: Impact of self monitoring of blood glucose in the management of patients with non-insu- lin treated diabetes: open parallel group randomised trial. BMJ 335:132, 2007

35. O'Kane MJ, Bunting B, Copeland M, Coates VE: Efficacy of self monitoring of blood glucose in patients with newly diagnosed type 2 diabetes (ESMON study): randomised controlled trial. BMJ 336:1174-1177, 2008

36. Simon J, Gray A, Clarke P, Wade A, Neil A, Farmer A: Cost effectiveness of self monitoring of blood glucose in patients with non-insulin treated type 2 diabetes: economic evaluation of data from the DiGEM trial. BMJ 336:1177-1180, 2008

37. Sacks DB, Bruns DE, Goldstein DE, Maclaren NK, McDonald JM, Parrott M: Guidelines and recommendations for laboratory analysis in the diagnosis and management of diabetes mellitus. Clin Chem 48:436-472, 2002

38. The Juvenile Diabetes Research Foundation Continuous Glucose Monitoring Study Group: Continuous glucose monitoring and intensive treatment of type 1 diabetes. N Engl J Med 359:1464-1476, 2008

39. Stratton IM, Adler AI, Neil HA, Matthews DR, Manley SE, Cull CA, Hadden D, Turner RC, Holman RR: Association of glycaemia with macrovascular and microvascular complications of type 2 diabetes (UKPDS 35): prospective observational study. BMJ 321:405-412, 2000

40. Cagliero E, Levina EV, Nathan DM: Immediate feedback of HbAlc levels improves glycemic control in type 1 and insulin-treated type 2 diabetic patients. Diabetes Care 22:1785-1789, 1999

41. Miller CD, Barnes CS, Phillips LS, Ziemer DC, Gallina DL, Cook CB, Maryman SD, El Kebbi IM: Rapid Alc availability improves clinical decision-making in an urban primary care clinic. Diabetes Care 26:1158-1163, 2003

42. Nathan DM, Kuenen J, Borg R, Zheng H, Schoenfeld D, Heine RJ: Translating the AlC assay into estimated average glucose values. Diabetes Care 31:14731478, 2008

43. Rohlfing CL, Wiedmeyer HM, Little RR, England JD, Tennill A, Goldstein DE: Defining the relationship between plasma glucose and $\mathrm{HbA}(1 \mathrm{c})$ : analysis of glucose profiles and $\mathrm{HbA}(1 \mathrm{c})$ in the Diabetes Control and Complications Trial. Diabetes Care 25:275-278, 2002

44. Wilson DM, Kollman: Relationship of AlC to glucose concentrations in children with type 1 diabetes: assessments by high-frequency glucose determinations by sensors. Diabetes Care 31:381385, 2008

45. The effect of intensive treatment of diabetes on the development and progression of long-term complications in insulin-dependent diabetes mellitus. The Diabetes Control and Complica- 
tions Trial Research Group. N Engl J Med 329:977-986, 1993

46. Retinopathy and nephropathy in patients with type 1 diabetes four years after a trial of intensive therapy: the Diabetes Control and Complications Trial/Epidemiology of Diabetes Interventions and Complications Research Group. N Engl J Med 342:381-389, 2000

47. Martin CL, Albers J, Herman WH, Cleary P, Waberski B, Greene DA, Stevens MJ, Feldman EL: Neuropathy among the diabetes control and complications trial cohort 8 years after trial completion. Diabetes Care 29:340-344, 2006

48. Ohkubo Y, Kishikawa H, Araki E, Miyata T, Isami S, Motoyoshi S, Kojima Y, Furuyoshi $\mathrm{N}$, Shichiri M: Intensive insulin therapy prevents the progression of diabetic microvascular complications in Japanese patients with non-insulindependent diabetes mellitus: a randomized prospective 6-year study. Diabetes Res Clin Pract 28:103-117, 1995

49. Effect of intensive blood-glucose control with metformin on complications in overweight patients with type 2 diabetes (UKPDS 34): UK Prospective Diabetes Study (UKPDS) Group. Lancet 352: 854-865, 1998

50. Intensive blood-glucose control with sulphonylureas or insulin compared with conventional treatment and risk of complications in patients with type 2 diabetes (UKPDS 33): UK Prospective Diabetes Study (UKPDS) Group. Lancet 352:837-853, 1998

51. Holman RR, Paul SK, Bethel MA, Matthews DR, Neil HA: 10-Year follow-up of intensive glucose control in type 2 diabetes. N Engl J Med 359:1577-1589, 2008

52. Lawson ML, Gerstein HC, Tsui E, Zinman B: Effect of intensive therapy on early macrovascular disease in young individuals with type 1 diabetes: a systematic review and meta-analysis. Diabetes Care 22 (Suppl. 2):B35-B39, 1999

53. Selvin E, Marinopoulos S, Berkenblit G, Rami T, Brancati FL, Powe NR, Golden SH: Meta-analysis: glycosylated hemoglobin and cardiovascular disease in diabetes mellitus. Ann Intern Med 141: 421-431, 2004

54. Stettler C, Allemann S, Juni P, Cull CA, Holman RR, Egger M, Krahenbuhl S, Diem P: Glycemic control and macrovascular disease in types 1 and 2 diabetes mellitus: meta-analysis of randomized trials. Am Heart J 152:27-38, 2006

55. Nathan DM, Cleary PA, Backlund JY, Genuth SM, Lachin JM, Orchard TJ, Raskin P, Zinman B: Intensive diabetes treatment and cardiovascular disease in patients with type 1 diabetes. $N$ Engl J Med 353:2643-2653, 2005

56. Gerstein HC, Miller ME, Byington RP,
Goff DC Jr, Bigger JT, Buse JB, Cushman WC, Genuth S, Ismail-Beigi F, Grimm RH Jr, Probstfield JL, Simons-Morton DG, Friedewald WT: Effects of intensive glucose lowering in type 2 diabetes. N Engl J Med 358:2545-2559, 2008

57. Patel A, MacMahon S, Chalmers J, Neal B, Billot L, Woodward M, Marre M, Cooper M, Glasziou P, Grobbee D, Hamet P, Harrap S, Heller S, Liu L, Mancia G, Mogensen CE, Pan C, Poulter N, Rodgers A, Williams B, Bompoint S, De Galan BE, Joshi R, Travert F: Intensive blood glucose control and vascular outcomes in patients with type 2 diabetes. $N$ Engl J Med 358:2560-2572, 2008

58. Duckworth W: VADT results. Presented at the 68th Annual Meeting of the American Diabetes Association, 6-10 June 2008, at the Moscone Convention Center, San Francisco, CA

59. American Diabetes Association: Postprandial blood glucose (Consensus Statement). Diabetes Care 24:775-778, 2001

60. Ceriello A, Taboga C, Tonutti L, Quagliaro L, Piconi L, Bais B, Da Ros R, Motz E: Evidence for an independent and cumulative effect of postprandial hypertriglyceridemia and hyperglycemia on endothelial dysfunction and oxidative stress generation: effects of short- and long-term simvastatin treatment. Circulation 106:1211-1218, 2002

61. Metzger BE, Buchanan TA, Coustan DR, de Leiva A, Dunger DB, Hadden DR, Hod M, Kitzmiller JL, Kjos SL, Oats JN, Pettitt DJ, Sacks DA, Zoupas C: Summary and recommendations of the Fifth International Workshop-Conference on Gestational Diabetes Mellitus. Diabetes Care 30 (Suppl. 2):S251-S260, 2007

62. Kitzmiller JL, Block JM, Brown FM, Catalano PM, Conway DL, Coustan DR, Gunderson EP, Herman WH, Hoffman LD, Inturrisi M, Jovanovic LB, Kjos SI, Knopp RH, Montoro MN, Ogata ES, Paramsothy P, Reader DM, Rosenn BM, Thomas AM, Kirkman MS: Managing preexisting diabetes for pregnancy: summary of evidence and consensus recommendations for care. Diabetes Care 31: 1060-1079, 2008

63. DeWitt DE, Hirsch IB: Outpatient insulin therapy in type 1 and type 2 diabetes mellitus: scientific review. JAMA 289: 2254-2264, 2003

64. Rosenstock J, Dailey G, Massi-Benedetti M, Fritsche A, Lin Z, Salzman A: Reduced hypoglycemia risk with insulin glargine: a meta-analysis comparing insulin glargine with human NPH insulin in type 2 diabetes. Diabetes Care 28:950955, 2005

65. Mooradian AD, Bernbaum M, Albert SG: Narrative review: a rational approach to starting insulin therapy. Ann Intern Med 145:125-134, 2006
66. Nathan DM, Buse JB, Davidson MB, Heine RJ, Holman RR, Sherwin R, Zinman B: Management of hyperglycemia in type 2 diabetes: a consensus algorithm for the initiation and adjustment of therapy: a consensus statement from the American Diabetes Association and the European Association for the Study of Diabetes. Diabetes Care 29:1963-1972, 2006

67. Nathan DM, Buse JB, Davidson MB, Ferrannini E, Holman RR, Sherwin R, and Zinman B: Medical management of hyperglycemia in type 2 diabetes: a consensus algorithm for the initiation and adjustment of therapy: a consensus statement of the American Diabetes Association and the European Association for the Study of Diabetes. Diabetes Care 32:193-203, 2009

68. American Diabetes Association: Nutrition recommendations and interventions for diabetes-2006. Diabetes Care 29:2140-2157, 2006

69. DAFNE Study Group: Training in flexible, intensive insulin management to enable dietary freedom in people with type 1 diabetes: dose adjustment for normal eating (DAFNE) randomised controlled trial. BMJ 325:746, 2002

70. Franz MJ, Monk A, Barry B, McClain K, Weaver T, Cooper N, Upham P, Bergenstal R, Mazze RS: Effectiveness of medical nutrition therapy provided by dietitians in the management of non-insulin-dependent diabetes mellitus: a randomized, controlled clinical trial. J Am Diet Assoc 95:1009-1017, 1995

71. Goldhaber-Fiebert JD, Goldhaber-Fiebert SN, Tristan ML, Nathan DM: Randomized controlled community-based nutrition and exercise intervention improves glycemia and cardiovascular risk factors in type 2 diabetic patients in rural Costa Rica. Diabetes Care 26:24-29, 2003

72. Lemon CC, Lacey K, Lohse B, Hubacher DO, Klawitter B, Palta M: Outcomes monitoring of health, behavior, and quality of life after nutrition intervention in adults with type 2 diabetes. J Am Diet Assoc 104:1805-1815, 2004

73. Miller CK, Edwards L, Kissling G, Sanville L: Nutrition education improves metabolic outcomes among older adults with diabetes mellitus: results from a randomized controlled trial. Prev Med 34:252-259, 2002

74. Wilson C, Brown T, Acton K, Gilliland S: Effects of clinical nutrition education and educator discipline on glycemic control outcomes in the Indian health service. Diabetes Care 26:2500-2504, 2003

75. Graber AL, Elasy TA, Quinn D, Wolff K, Brown A: Improving glycemic control in adults with diabetes mellitus: shared responsibility in primary care practices. South Med J 95:684-690, 2002 
76. Gaetke LM, Stuart MA, Truszczynska H: A single nutrition counseling session with a registered dietitian improves short-term clinical outcomes for rural Kentucky patients with chronic diseases. J Am Diet Assoc 106:109-112, 2006

77. Yu-Poth S, Zhao G, Etherton T, Naglak M, Jonnalagadda S, Kris-Etherton PM: Effects of the National Cholesterol Education Program's Step I and Step II dietary intervention programs on cardiovascular disease risk factors: a meta-analysis. Am J Clin Nutr 69:632646, 1999

78. Van Horn L, McCoin M, Kris-Etherton PM, Burke F, Carson JA, Champagne CM, Karmally W, Sikand G: The evidence for dietary prevention and treatment of cardiovascular disease. J Am Diet Assoc 108:287-331, 2008

79. Appel LJ, Moore TJ, Obarzanek E, Vollmer WM, Svetkey LP, Sacks FM, Bray GA, Vogt TM, Cutler JA, Windhauser MM, Lin PH, Karanja N: A clinical trial of the effects of dietary patterns on blood pressure: DASH Collaborative Research Group. N Engl J Med 336:1117-1124, 1997

80. Norris SL, Zhang X, Avenell A, Gregg E, Bowman B, Schmid CH, Lau J: Longterm effectiveness of weight-loss interventions in adults with pre-diabetes: a review. Am J Prev Med 28:126-139, 2005

81. Klein S, Sheard NF, Pi-Sunyer X, Daly A, Wylie-Rosett J, Kulkarni K, Clark NG: Weight management through lifestyle modification for the prevention and management of type 2 diabetes: rationale and strategies: a statement of the American Diabetes Association, the North American Association for the Study of Obesity, and the American Society for Clinical Nutrition. Diabetes Care 27:2067-2073, 2004

82. Norris SL, Zhang X, Avenell A, Gregg E, Schmid CH, Kim C, Lau J: Efficacy of pharmacotherapy for weight loss in adults with type 2 diabetes mellitus: a meta-analysis. Arch Intern Med 164: 1395-1404, 2004

83. Wolf AM, Conaway MR, Crowther JQ, Hazen KY, Nadler L, Oneida B, Bovbjerg VE: Translating lifestyle intervention to practice in obese patients with type 2 diabetes: Improving Control with Activity and Nutrition (ICAN) study. Diabetes Care 27:1570-1576, 2004

84. Manning RM, Jung RT, Leese GP, Newton RW: The comparison of four weight reduction strategies aimed at overweight patients with diabetes mellitus: fouryear follow-up. Diabet Med 15:497-502, 1998

85. Shai I, Schwarzfuchs D, Henkin Y, Shahar DR, Witkow S, Greenberg I, Golan R, Fraser D, Bolotin A, Vardi H, TangiRozental O, Zuk-Ramot R, Sarusi B,
Brickner D, Schwartz Z, Sheiner E, Marko R, Katorza E, Thiery J, Fiedler GM, Bluher M, Stumvoll M, Stampfer MJ: Weight loss with a low-carbohydrate, Mediterranean, or low-fat diet. N Engl J Med 359:229-241, 2008

86. Franz MJ, VanWormer JJ, Crain AL, Boucher JL, Histon T, Caplan W, Bowman JD, Pronk NP: Weight-loss outcomes: a systematic review and metaanalysis of weight-loss clinical trials with a minimum 1-year follow-up. J Am Diet Assoc 107:1755-1767, 2007

87. Pi-Sunyer X, Blackburn G, Brancati FL, Bray GA, Bright R, Clark JM, Curtis JM, Espeland MA, Foreyt JP, Graves K, Haffner SM, Harrison B, Hill JO, Horton ES, Jakicic J, Jeffery RW, Johnson KC, Kahn S, Kelley DE, Kitabchi AE, Knowler WC, Lewis CE, Maschak-Carey BJ, Montgomery B, Nathan DM, Patricio J, Peters A, Redmon JB, Reeves RS, Ryan DH, Safford M, Van Dorsten B, Wadden TA, Wagenknecht L, Wesche-Thobaben J, Wing RR, Yanovski SZ: Reduction in weight and cardiovascular disease risk factors in individuals with type 2 diabetes: 1-year results of the Look AHEAD trial. Diabetes Care 30:1374-1383, 2007

88. Foster GD, Wyatt HR, Hill JO, McGuckin BG, Brill C, Mohammed BS, Szapary PO, Rader DJ, Edman JS, Klein $S$ : A randomized trial of a low-carbohydrate diet for obesity. N Engl J Med 348: 2082-2090, 2003

89. Stern L, Iqbal N, Seshadri P, Chicano KL, Daily DA, McGrory J, Williams M, Gracely EJ, Samaha FF: The effects of low-carbohydrate versus conventional weight loss diets in severely obese adults: one-year follow-up of a randomized trial. Ann Intern Med 140:778-785, 2004

90. Gardner C, Kiazand A, Alhassan S, Soowon K, Stafford R, Balise R, Kraemer $\mathrm{H}$, King A: Comparison of the Atkins, Zone, Ornish, and LEARN diets for change in weight and related risk factors among overweight premenopausal women JAMA 297:969-977, 2007

91. Nordmann AJ, Nordmann A, Briel M, Keller U, Yancy WS Jr, Brehm BJ, Bucher HC: Effects of low-carbohydrate vs lowfat diets on weight loss and cardiovascular risk factors: a meta-analysis of randomized controlled trials. Arch Intern Med 166:285-293, 2006

92. Institute of Medicine: Dietary Reference Intakes: Energy, Carbohydrate, Fiber, Fat, Fatty Acids, Cholesterol, Protein, and Amino Acids. Washington, D.C., National Academies Press, 2002

93. Barnard ND, Cohen J, Jenkins DJ, Turner-McGrievy G, Gloede L, Jaster B, Seidl K, Green AA, Talpers S: A low-fat vegan diet improves glycemic control and cardiovascular risk factors in a randomized clinical trial in individuals with type 2 diabetes. Diabetes Care 29:1777-1783, 2006

94. Turner-McGrievy GM, Barnard ND, Cohen J, Jenkins DJ, Gloede L, Green AA: Changes in nutrient intake and dietary quality among participants with type 2 diabetes following a low-fat vegan diet or a conventional diabetes diet for 22 weeks. J Am Diet Assoc 108:1636-1645, 2008

95. Franz MJ, Bantle JP, Beebe CA, Brunzell JD, Chiasson JL, Garg A, Holzmeister LA, Hoogwerf B, Mayer-Davis E, Mooradian AD, Purnell JQ, Wheeler M: Evidence-based nutrition principles and recommendations for the treatment and prevention of diabetes and related complications. Diabetes Care 25:148-198, 2002

96. Buchwald H, Estok R, Fahrbach K, Banel $\mathrm{D}$, Jensen MD, Pories WJ, Bantle JP, Sledge I: Weight and type 2 diabetes after bariatric surgery: systematic review and meta-analysis. Am J Med. In Press

97. Dixon JB, O'Brien PE, Playfair J, Chapman L, Schachter LM, Skinner S, Proietto J, Bailey M, Anderson M: Adjustable gastric banding and conventional therapy for type 2 diabetes: a randomized controlled trial. JAMA 299:316-323, 2008

98. Buchwald H, Estok R, Fahrbach K, Banel $\mathrm{D}$, Sledge I: Trends in mortality in bariatric surgery: a systematic review and meta-analysis. Surgery 142:621-632, 2007

99. Sjostrom L, Narbro K, Sjostrom CD, Karason K, Larsson B, Wedel H, Lystig T, Sullivan M, Bouchard C, Carlsson B, Bengtsson C, Dahlgren S, Gummesson A, Jacobson P, Karlsson J, Lindroos AK, Lonroth H, Naslund I, Olbers T, Stenlof K, Torgerson J, Agren G, Carlsson LM: Effects of bariatric surgery on mortality in Swedish obese subjects. N Engl J Med 357:741-752, 2007

100. Piette JD, Glasgow RE: Sttategies for improving behavioral and health outcomes among people with diabetes: self managemnt education. In Evidence-Based Diabetes Care. Gerstein HC, Hayes RB, Eds. Ontario, Canada, BC Decker, 2000

101. Norris SL, Engelgau MM, Narayan KM: Effectiveness of self-management training in type 2 diabetes: a systematic review of randomized controlled trials. Diabetes Care 24:561-587, 2001

102. Norris SL, Lau J, Smith SJ, Schmid CH, Engelgau MM: Self-management education for adults with type 2 diabetes: a meta-analysis of the effect on glycemic control. Diabetes Care 25:1159-1171, 2002

103. Gary TL, Genkinger JM, Guallar E, Peyrot M, Brancati FL: Meta-analysis of randomized educational and behavioral interventions in type 2 diabetes. Diabetes 
Educ 29:488-501, 2003

104. Steed L, Cooke D, Newman S: A systematic review of psychosocial outcomes following education, self-management and psychological interventions in diabetes mellitus. Patient Educ Couns 51:515, 2003

105. Ellis SE, Speroff T, Dittus RS, Brown A, Pichert JW, Elasy TA: Diabetes patient education: a meta-analysis and meta-regression. Patient Educ Couns 52:97-105, 2004

106. Warsi A, Wang PS, LaValley MP, Avorn J, Solomon DH: Self-management education programs in chronic disease: a systematic review and methodological critique of the literature. Arch Intern Med 164:1641-1649, 2004

107. Funnell MM, Brown TL, Childs BP, Haas LB, Hosey GM, Jensen B, Maryniuk M, Peyrot M, Piette JD, Reader D, Siminerio LM, Weinger K, Weiss MA: National standards for diabetes self-management education. Diabetes Care 30:16301637,2007

108. Mulcahy K, Maryniuk M, Peeples M, Peyrot M, Tomky D, Weaver T, Yarborough P: Diabetes self-management education core outcomes measures. Diabetes Educ 29: 768- 84:787, 2003

109. Glasgow RE, Peeples M, Skovlund SE: Where is the patient in diabetes performance measures? The case for including patient-centered and self-management measures. Diabetes Care 31:1046-1050, 2008

110. Barker JM, Goehrig SH, Barriga K, Hoffman M, Slover R, Eisenbarth GS, Norris JM, Klingensmith GJ, Rewers M: Clinical characteristics of children diagnosed with type 1 diabetes through intensive screening and follow-up. Diabetes Care 27:1399-1404, 2004

111. Rickheim PL, Weaver TW, Flader JL, Kendall DM: Assessment of group versus individual diabetes education: a randomized study. Diabetes Care 25:269274, 2002

112. Trento M, Passera P, Borgo E, Tomalino M, Bajardi M, Cavallo F, Porta M: A 5-year randomized controlled study of learning, problem solving ability, and quality of life modifications in people with type 2 diabetes managed by group care. Diabetes Care 27:670-675, 2004

113. Norris SL, Chowdhury FM, Van Le K, Horsley T, Brownstein JN, Zhang X, Jack L Jr, Satterfield DW: Effectiveness of community health workers in the care of persons with diabetes. Diabet Med 23: 544-556, 2006

114. Sigal RJ, Kenny GP, Wasserman DH, Castaneda-Sceppa C: Physical activity/ exercise and type 2 diabetes. Diabetes Care 27:2518-2539, 2004

115. Wasserman DH, Zinman B: Exercise in individuals with IDDM. Diabetes Care $17: 924-937,1994$
116. Boulé NG, Haddad E, Kenny GP, Wells GA, Sigal RJ: Effects of exercise on glycemic control and body mass in type 2 diabetes mellitus: a meta-analysis of controlled clinical trials. JAMA 286: 1218-1227, 2001

117. Boulé NG, Kenny GP, Haddad E, Wells GA, Sigal RJ: Meta-analysis of the effect of structured exercise training on cardiorespiratory fitness in type 2 diabetes mellitus. Diabetologia 46:1071-1081, 2003

118. US Department of Health and Human Services: Physical Activity and Health: A Report of the Surgeon General. Atlanta, GA, Centers for Disease Control and Prevention, 1996

119. Ivy JL: Role of exercise training in the prevention and treatment of insulin resistance and non-insulin-dependent diabetes mellitus. Sports Med 24:321-336, 1997

120. Dunstan DW, Daly RM, Owen N, Jolley D, de Court, Shaw J, Zimmet P: Highintensity resistance training improves glycemic control in older patients with type 2 diabetes. Diabetes Care 25:1729_ 1736, 2002

121. Castaneda C, Layne JE, Munoz-Orians L, Gordon PL, Walsmith J, Foldvari M, Roubenoff R, Tucker KL, Nelson ME: A randomized controlled trial of resistance exercise training to improve glycemic control in older adults with type 2 diabetes. Diabetes Care 25:2335-2341, 2002

122. Sigal RJ, Kenny GP, Boule NG, Wells GA, Prud'homme D, Fortier M, Reid RD, Tulloch H, Coyle D, Phillips P, Jennings A, Jaffey J: Effects of aerobic training, resistance training, or both on glycemic control in type 2 diabetes: a randomized trial. Ann Intern Med 147:357-369, 2007

123. Bax JJ, Young LH, Frye RL, Bonow RO, Steinberg HO, Barrett EJ: Screening for coronary artery disease in patients with diabetes. Diabetes Care 30:2729-2736, 2007

124. Berger M, Berchtold P, Cuppers HJ, Drost H, Kley HK, Muller WA, Wiegelmann W, Zimmerman-Telschow H, Gries FA, Kruskemper HL, Zimmermann H: Metabolic and hormonal effects of muscular exercise in juvenile type diabetics. Diabetologia 13:355-365, 1977

125. American Diabetes Association: Physical activity/exercise and diabetes (Position Statement). Diabetes Care 27 (Suppl. 1): S58-S62, 2004

126. Berger M: Adjustment of insulin and oral agent therapy. In Handbook of Exercise in Diabetes. 2nd ed. Ruderman N, Devlin JTSSH, Krisska A, Eds. Alexandria, VA, American Diabetes Association, 2002, p. 365-376

127. Aiello LP, Wong J, Cavallerano J, Bursell SE, Aiello LM: Retinopathy. In Handbook of Exercise in Diabetes. 2nd ed. Ruderman N, Devlin JT, Kriska A, Eds. Alexandria, VA, American Diabetes Association, 2002, p. 401-413

128. Vinik A, Erbas T: Neuropathy. In Handbook of Exercise in Diabetes. 2nd ed. Ruderman N, Devlin JT, Kriska A, Eds. Alexnadria, VA, Anerican Diabetes Association, 2002, p. 463-496

129. Levin ME: The Diabetic Foot. In Handbook of Exercise in Diabetes. Ruderman N, Devlin JT, Kriska A, Eds. Alexandria, VA, American Diabetes Association, 2002, p. 385-399

130. Wackers FJ, Young LH, Inzucchi SE, Chyun DA, Davey JA, Barrett EJ, Taillefer R, Wittlin SD, Heller GV, Filipchuk N, Engel S, Ratner RE, Iskandrian AE: Detection of silent myocardial ischemia in asymptomatic diabetic subjects: the DIAD study. Diabetes Care 27:19541961, 2004

131. Valensi P, Sachs RN, Harfouche B, Lormeau B, Paries J, Cosson E, Paycha F, Leutenegger M, Attali JR: Predictive value of cardiac autonomic neuropathy in diabetic patients with or without silent myocardial ischemia. Diabetes Care 24:339-343, 2001

132. Mogensen CE: Nephropathy. In Handbook of Exercise in Diabetes. 2nd ed. Ruderman N, Devlin JT, Kriska A, Eds. Alexandria, VA, American Diabetes Association, 2002, p. 433-449

133. Anderson RJ, Grigsby AB, Freedland KE, de Groot M, McGill JB, Clouse RE, Lustman PJ: Anxiety and poor glycemic control: a meta-analytic review of the literature. Int J Psychiatry Med 32:235247, 2002

134. Jacobson AM: Depression and diabetes. Diabetes Care 16:1621-1623, 1993

135. Rubin RR, Peyrot M: Psychosocial problems and interventions in diabetes: a review of the literature. Diabetes Care 15: 1640-1657, 1992

136. Surwit RS, Schneider MS, Feinglos MN: Stress and diabetes mellitus. Diabetes Care 15:1413-1422, 1992

137. Young-Hyman D: Psycosocial factors affecting adherence, quality of life, and well-being: Helping patients cope. In Medical Management of Type 1 Diabetes. 4th ed. Bode B, Ed. Alexandria, VA, American Diabetes Association, 2004, p. 162-182

138. Delahanty LM, Grant RW, Wittenberg E, Bosch JL, Wexler DJ, Cagliero E, Meigs JB: Association of diabetes-related emotional distress with diabetes treatment in primary care patients with type 2 diabetes. Diabet Med 24:48-54, 2007

139. Anderson BJ, Auslander WF, Jung KC, Miller JP, Santiago JV: Assessing family sharing of diabetes responsibilities. J Pediatr Psychol 15:477-492, 1990

140. McCulloch DK, Glasgow RE, Hampson SE, Wagner E: A systematic approach to 
diabetes management in the post-DCCT era. Diabetes Care 17:765-769, 1994

141. Rubin RR, Peyrot M: Psychological issues and treatments for people with diabetes. J ClinPsychol 57:457-478, 2001

142. Peyrot M, Rubin RR: Behavioral and psychosocial interventions in diabetes: a conceptual review. Diabetes Care 30: 2433-2440, 2007

143. Lustman PJ, Griffith LS, Clouse RE, Cryer PE: Psychiatric illness in diabetes mellitus: relationship to symptoms and glucose control. J Nerv Ment Dis 174:736-742, 1986

144. Marcus MD, Wing RR: Eating disorders and diabetes. In Neuropsychological and Behavioral Aspects of Diabetes. Holmes CS, Ed. New York, Springer-Verlag, 1990, p. 102-121

145. American Diabetes Association: Hyperglycemic crises in diabetes. Diabetes Care 27 (Suppl. 1):S94-S102, 2004

146. Cryer PE: Hypoglycaemia: the limiting factor in the glycaemic management of type I and type II diabetes. Diabetologia 45:937-948, 2002

147. Gannon MC, Nuttall FQ: Protein and diabetes. In American Diabetes Association Guide to Medical Nutrition Therapy for Diabetes. Franz MJ, Bantle JP, Eds. Alexandria, VA, American Diabetes Association, 1999, p. 107-125

148. Cryer PE: Diverse causes of hypoglycemia-associated autonomic failure in diabetes. N Engl J Med 350:2272-2279, 2004

149. Cryer PE, Davis SN, Shamoon H: Hypoglycemia in diabetes. Diabetes Care 26: 1902-1912, 2003

150. Smith SA, Poland GA: Use of influenza and pneumococcal vaccines in people with diabetes. Diabetes Care 23:95-108, 2000

151. Colquhoun AJ, Nicholson KG, Botha JL, Raymond NT: Effectiveness of influenza vaccine in reducing hospital admissions in people with diabetes. Epidemiol Infect 119:335-341, 1997

152. Bridges CB, Fukuda K, Uyeki TM, Cox NJ, Singleton JA: Prevention and control of influenza: recommendations of the Advisory Committee on Immunization Practices (ACIP). MMWR Recomm Rep 51:1-31, 2002

153. American Diabetes Association: Influenza and pneumococcal immunization in diabetes (Position Statement). Diabetes Care 27 (Suppl. 1):S111-S113, 2004

154. Gaede P, Lund-Andersen H, Parving $\mathrm{HH}$, Pedersen O: Effect of a multifactorial intervention on mortality in type 2 diabetes. N Engl J Med 358:580-591, 2008

155. Arauz-Pacheco C, Parrott MA, Raskin P: The treatment of hypertension in adult patients with diabetes. Diabetes Care 25: $134-147,2002$

156. Haffner SM: Management of dyslipide- mia in adults with diabetes. Diabetes Care 21:160-178, 1998

157. Colwell JA: Aspirin therapy in diabetes. Diabetes Care 20:1767-1771, 1997

158. Haire-Joshu D, Glasgow RE, Tibbs TL: Smoking and diabetes. Diabetes Care 22: 1887-1898, 1999

159. Buse JB, Ginsberg HN, Barkis GL, Clark NG, Costa F, Eckel R, Fonseca V, Gerstein HC, Grundy S, Nesto RW, Pignone MP, Plutzky J, Porte D, Redberg R, Stitzel KF, Stone NJ: Primary prevention of cardiovascular diseases in people with diabetes mellitus: a scientific statement from the American Heart Association and the American Diabetes Association. Diabetes Care 30:162-172, 2007

160. Chobanian AV, Bakris GL, Black HR, Cushman WC, Green LA, Izzo JL Jr, Jones DW, Materson BJ, Oparil S, Wright JT Jr, Roccella EJ: The Seventh Report of the Joint National Committee on Prevention, Detection, Evaluation, and Treatment of High Blood Pressure: the JNC 7 report. JAMA 289:25602572, 2003

161. Bobrie G, Chatellier G, Genes N, Clerson P, Vaur L, Vaisse B, Menard J, Mallion JM: Cardiovascular prognosis of "masked hypertension" detected by blood pressure self-measurement in elderly treated hypertensive patients. JAMA 291:1342-1349, 2004

162. Sega R, Facchetti R, Bombelli M, Cesana G, Corrao G, Grassi G, Mancia G: Prognostic value of ambulatory and home blood pressures compared with office blood pressure in the general population: follow-up results from the Pressioni Arteriose Monitorate e Loro Associazioni (PAMELA) study. Circulation 111:1777-1783, 2005

163. Tight blood pressure control and risk of macrovascular and microvascular complications in type 2 diabetes: UKPDS 38 : UK Prospective Diabetes Study Group. BMJ 317:703-713, 1998

164. Hansson L, Zanchetti A, Carruthers SG, Dahlof B, Elmfeldt D, Julius S, Menard J, Rahn KH, Wedel H, Westerling S: Effects of intensive blood-pressure lowering and low-dose aspirin in patients with hypertension: principal results of the Hypertension Optimal Treatment (HOT) randomised trial: HOT Study Group. Lancet 351:1755-1762, 1998

165. Adler AI, Stratton IM, Neil HA, Yudkin JS, Matthews DR, Cull CA, Wright AD, Turner RC, Holman RR: Association of systolic blood pressure with macrovascular and microvascular complications of type 2 diabetes (UKPDS 36): prospective observational study. BMJ 321:412419, 2000

166. Lewington S, Clarke R, Qizilbash N, Peto R, Collins R: Age-specific relevance of usual blood pressure to vascular mortality: a meta-analysis of individual data for one million adults in 61 prospective studies. Lancet 360:1903-1913, 2002

167. Stamler J, Vaccaro O, Neaton JD, Wentworth D: Diabetes, other risk factors, and 12-yr cardiovascular mortality for men screened in the Multiple Risk Factor Intervention Trial. Diabetes Care 16: 434-444, 1993

168. Sacks FM, Svetkey LP, Vollmer WM, Appel LJ, Bray GA, Harsha D, Obarzanek E, Conlin PR, Miller ER III, Simons-Morton DG, Karanja N, Lin PH: Effects on blood pressure of reduced dietary sodium and the Dietary Approaches to Stop Hypertension (DASH) diet: DASH-Sodium Collaborative Research Group. N Engl J Med 344:3-10, 2001

169. Tatti P, Pahor M, Byington RP, Di Mauro P, Guarisco R, Strollo G, Strollo F: Outcome results of the Fosinopril Versus Amlodipine Cardiovascular Events Randomized Trial (FACET) in patients with hypertension and NIDDM. Diabetes Care 21:597-603, 1998

170. Estacio RO, Jeffers BW, Hiatt WR, Biggerstaff SL, Gifford N, Schrier RW: The effect of nisoldipine as compared with enalapril on cardiovascular outcomes in patients with non-insulin-dependent diabetes and hypertension. N Engl J Med 338:645-652, 1998

171. Schrier RW, Estacio RO, Mehler PS, Hiatt WR: Appropriate blood pressure control in hypertensive and normotensive type 2 diabetes mellitus: a summary of the ABCD trial. Nat Clin Pract Nephrol 3:428-438, 2007

172. Major outcomes in high-risk hypertensive patients randomized to angiotensinconverting enzyme inhibitor or calcium channel blocker vs diuretic: the Antihypertensive and Lipid-Lowering Treatment to Prevent Heart Attack Trial (ALLHAT). JAMA 288:2981-2997, 2002

173. Psaty BM, Lumley T, Furberg CD, Schellenbaum G, Pahor M, Alderman MH, Weiss NS: Health outcomes associated with various antihypertensive therapies used as first-line agents: a network metaanalysis. JAMA 289:2534-2544, 2003

174. Effects of ramipril on cardiovascular and microvascular outcomes in people with diabetes mellitus: results of the HOPE study and MICRO-HOPE substudy: Heart Outcomes Prevention Evaluation Study Investigators. Lancet 355:253259, 2000

175. Pfeffer MA, Swedberg K, Granger CB, Held P, McMurray JJ, Michelson EL, Olofsson B, Ostergren J, Yusuf S, Pocock $S$ : Effects of candesartan on mortality and morbidity in patients with chronic heart failure: the CHARM-Overall programme. Lancet 362:759-766, 2003

176. Granger CB, McMurray JJ, Yusuf S, Held P, Michelson EL, Olofsson B, Ostergren J, Pfeffer MA, Swedberg K: Effects of can- 
desartan in patients with chronic heart failure and reduced left-ventricular systolic function intolerant to angiotensinconverting-enzyme inhibitors: the CHARM- Alternative trial. Lancet 362: 772-776,2003

177. McMurray JJ, Ostergren J, Swedberg K, Granger CB, Held P, Michelson EL, Olofsson B, Yusuf S, Pfeffer MA: Effects of candesartan in patients with chronic heart failure and reduced left-ventricular systolic function taking angiotensinconverting-enzyme inhibitors: the CHARM-Added trial. Lancet 362:767771, 2003

178. Lindholm LH, Ibsen H, Dahlof B, Devereux RB, Beevers $G$, de Faire U, Fyhrquist F, Julius S, Kjeldsen SE, Kristiansson K, Lederballe-Pedersen $\mathrm{O}$, Nieminen MS, Omvik P, Oparil S, Wedel H, Aurup P, Edelman J, Snapinn $S$ : Cardiovascular morbidity and mortality in patients with diabetes in the Losartan Intervention For Endpoint reduction in hypertension study (LIFE): a randomised trial against atenolol. Lancet 359: 1004-1010, 2002

179. Berl T, Hunsicker LG, Lewis JB, Pfeffer MA, Porush JG, Rouleau JL, Drury PL, Esmatjes E, Hricik D, Parikh CR, Raz I, Vanhille P, Wiegmann TB, Wolfe BM, Locatelli F, Goldhaber SZ, Lewis EJ: Cardiovascular outcomes in the Irbesartan Diabetic Nephropathy Trial of patients with type 2 diabetes and overt nephropathy. Ann Intern Med 138:542-549, 2003

180. Laffel LM, McGill JB, Gans DJ: The beneficial effect of angiotensin-converting enzyme inhibition with captopril on diabetic nephropathy in normotensive IDDM patients with microalbuminuria: North American Microalbuminuria Study Group. Am J Med 99:497-504, 1995

181. Bakris GL, Williams M, Dworkin L, Elliott WJ, Epstein M, Toto R, Tuttle K, Douglas J, Hsueh W, Sowers J: Preserving renal function in adults with hypertension and diabetes: a consensus approach: National Kidney Foundation Hypertension and Diabetes Executive Committees Working Group. AmJ Kidney Dis 36:646-661, 2000

182. Psaty BM, Smith NL, Siscovick DS, Koepsell TD, Weiss NS, Heckbert SR, Lemaitre RN, Wagner EH, Furberg CD: Health outcomes associated with antihypertensive therapies used as first-line agents: a systematic review and meta-analysis. JAMA 277:739-745, 1997

183. Patel A, MacMahon S, Chalmers J, Neal B, Woodward M, Billot L, Harrap S, Poulter N, Marre M, Cooper M, Glasziou P, Grobbee DE, Hamet P, Heller S, Liu LS, Mancia G, Mogensen CE, Pan CY, Rodgers A, Williams B: Effects of a fixed combination of perindopril and indap- amide on macrovascular and microvascular outcomes in patients with type 2 diabetes mellitus (the ADVANCE trial): a randomised controlled trial. Lancet 370:829-840, 2007

184. Sibai BM: Treatment of hypertension in pregnant women. N Engl J Med 335:257265, 1996

185. Baigent C, Keech A, Kearney PM, Blackwell L, Buck G, Pollicino C, Kirby A, Sourjina T, Peto R, Collins R, Simes R: Efficacy and safety of cholesterol-lowering treatment: prospective meta-analysis of data from 90,056 participants in 14 randomised trials of statins. Lancet 366 : 1267-1278, 2005

186. Pyorala K, Pedersen TR, Kjekshus J, Faergeman O, Olsson AG, Thorgeirsson G: Cholesterol lowering with simvastatin improves prognosis of diabetic patients with coronary heart disease: a subgroup analysis of the Scandinavian Simvastatin Survival Study (4S). Diabetes Care 20:614-620, 1997

187. Heart Protection Study Collaborative Group: MRC/BHF Heart Protection Study of cholesterol-lowering with simvastatin in 5963 people with diabetes: a randomised placebo-controlled trial. Lancet 361:2005-2016, 2003

188. Goldberg RB, Mellies MJ, Sacks FM, Moye LA, Howard BV, Howard WJ, Davis BR, Cole TG, Pfeffer MA, Braunwald E: Cardiovascular events and their reduction with pravastatin in diabetic and glucose-intolerant myocardial infarction survivors with average cholesterol levels: subgroup analyses in the cholesterol and recurrent events (CARE) trial: the Care Investigators. Circulation 98:2513-2519, 1998

189. Shepherd J, Barter P, Carmena R, Deedwania P, Fruchart JC, Haffner S, Hsia J, Breazna A, LaRosa J, Grundy S, Waters D: Effect of lowering LDL cholesterol substantially below currently recommended levels in patients with coronary heart disease and diabetes: the Treating to New Targets (TNT) study. Diabetes Care 29:1220-1226, 2006

190. Sever PS, Poulter NR, Dahlof B, Wedel H, Collins R, Beevers G, Caulfield M, Kjeldsen SE, Kristinsson A, McInnes GT, Mehlsen J, Nieminen M, O'Brien E, Ostergren J: Reduction in cardiovascular events with atorvastatin in 2,532 patients with type 2 diabetes: Anglo-Scandinavian Cardiac Outcomes TrialLipid-Lowering Arm (ASCOT-LLA). Diabetes Care 28:1151-1157, 2005

191. Knopp RH, d'Emden M, Smilde JG, Pocock SJ: Efficacy and safety of atorvastatin in the prevention of cardiovascular end points in subjects with type 2 diabetes: the Atorvastatin Study for Prevention of Coronary Heart Disease Endpoints in Non-insulin-dependent diabetes mellitus (ASPEN). Diabetes
Care 29:1478-1485, 2006

192. Singh IM, Shishehbor MH, Ansell BJ: High-density lipoprotein as a therapeutic target: a systematic review. JAMA 298:786-798, 2007

193. Singh IM, Shishehbor MH, Ansell BJ: High-density lipoprotein as a therapeutic target: a systematic review. JAMA 298:786-798, 2007

194. Canner PL, Berge KG, Wenger NK, Stamler J, Friedman L, Prineas RJ, Friedewald W: Fifteen year mortality in Coronary Drug Project patients: longterm benefit with niacin. J Am Coll Cardiol 8:1245-1255, 1986

195. Rubins HB, Robins SJ, Collins D, Fye CL, Anderson JW, Elam MB, Faas FH, Linares E, Schaefer EJ, Schectman G, Wilt TJ, Wittes J: Gemfibrozil for the secondary prevention of coronary heart disease in men with low levels of high-density lipoprotein cholesterol: Veterans Affairs High-Density Lipoprotein Cholesterol Intervention Trial Study Group. N Engl J Med 341:410-418, 1999

196. Frick MH, Elo O, Haapa K, Heinonen OP, Heinsalmi P, Helo P, Huttunen JK, Kaitaniemi P, Koskinen P, Manninen V: Helsinki Heart Study: primary-prevention trial with gemfibrozil in middleaged men with dyslipidemia: safety of treatment, changes in risk factors, and incidence of coronary heart disease. N Engl J Med 317:1237-1245, 1987

197. Keech A, Simes RJ, Barter P, Best J, Scott R, Taskinen MR, Forder P, Pillai A, Davis T, Glasziou P, Drury P, Kesaniemi YA, Sullivan D, Hunt D, Colman P, d'Emden M, Whiting M, Ehnholm C, Laakso M: Effects of long-term fenofibrate therapy on cardiovascular events in 9795 people with type 2 diabetes mellitus (the FIELD study): randomised controlled trial. Lancet 366:1849-1861, 2005

198. Executive Summary of The Third Report of The National Cholesterol Education Program (NCEP) Expert Panel on Detection, Evaluation, And Treatment of High Blood Cholesterol In Adults (Adult Treatment Panel III). JAMA 285:24862497, 2001

199. Hayward RA, Hofer TP, Vijan S: Narrative review: lack of evidence for recommended low-density lipoprotein treatment targets: a solvable problem. Ann Intern Med 145:520-530, 2006

200. Cannon CP, Braunwald E, McCabe CH, Rader DJ, Rouleau JL, Belder R, Joyal SV, Hill KA, Pfeffer MA, Skene AM: Intensive versus moderate lipid lowering with statins after acute coronary syndromes. N Engl J Med 350:1495-1504, 2004

201. de Lemos JA, Blazing MA, Wiviott SD, Lewis EF, Fox KA, White HD, Rouleau JL, Pedersen TR, Gardner LH, Mukherjee R, Ramsey KE, Palmisano J, Bilheimer DW, Pfeffer MA, Califf RM, Braunwald E: Early intensive vs a de- 
layed conservative simvastatin strategy in patients with acute coronary syndromes: phase $\mathrm{Z}$ of the $\mathrm{A}$ to $\mathrm{Z}$ trial. JAMA 292:1307-1316, 2004

202. Nissen SE, Tuzcu EM, Schoenhagen P, Brown BG, Ganz P, Vogel RA, Crowe T, Howard G, Cooper CJ, Brodie B, Grines CL, DeMaria AN: Effect of intensive compared with moderate lipid-lowering therapy on progression of coronary atherosclerosis: a randomized controlled trial. JAMA 291:1071-1080, 2004

203. Grundy SM, Cleeman JI, Merz CN, Brewer HB Jr, Clark LT, Hunninghake DB, Pasternak RC, Smith SC Jr, Stone NJ: Implications of recent clinical trials for the National Cholesterol Education Program Adult Treatment Panel III guidelines. Circulation 110:227-239, 2004

204. Chasman DI, Posada D, Subrahmanyan L, Cook NR, Stanton VP Jr, Ridker PM: Pharmacogenetic study of statin therapy and cholesterol reduction. JAMA 291:2821-2827, 2004

205. Elam MB, Hunninghake DB, Davis KB, Garg R, Johnson C, Egan D, Kostis JB, Sheps DS, Brinton EA: Effect of niacin on lipid and lipoprotein levels and glycemic control in patients with diabetes and peripheral arterial disease: the ADMIT study: a randomized trial: Arterial Disease Multiple Intervention Trial. JAMA 284:1263-1270, 2000

206. Grundy SM, Vega GL, McGovern ME, Tulloch BR, Kendall DM, Fitz-Patrick D, Ganda OP, Rosenson RS, Buse JB, Robertson DD, Sheehan JP: Efficacy, safety, and tolerability of once-daily niacin for the treatment of dyslipidemia associated with type 2 diabetes: results of the assessment of diabetes control and evaluation of the efficacy of niaspan trial. Arch Intern Med 162:1568-1576, 2002

207. Jones PH, and Davidson MH Reporting rate of rhabdomyolysis with fenofibrate + statin versus gemfibrozil + any statin. Am J Cardiol 95:120-122, 2005

208. Brunzell JD, Davidson M, Furberg CD, Goldberg RB, Howard BV, Stein JH, Witztum JL: Lipoprotein management in patients with cardiometabolic risk: consensus statement from the American Diabetes Association and the American College of Cardiology Foundation. Diabetes Care 31:811-822, 2008

209. Colhoun HM, Betteridge DJ, Durrington PN, Hitman GA, Neil HA, Livingstone SJ, Thomason MJ, Mackness MI, Charlton-Menys V, Fuller JH: Primary prevention of cardiovascular disease with atorvastatin in type 2 diabetes in the Collaborative Atorvastatin Diabetes Study (CARDS): multicentre randomised placebo-controlled trial. Lancet 364:685696, 2004

210. American Diabetes Association: Aspirin therapy in diabetes (Position Statement).
Diabetes Care 27 (Suppl. 1):S72-S73, 2004

211. Hayden M, Pignone M, Phillips C, Mulrow C: Aspirin for the primary prevention of cardiovascular events: a summary of the evidence for the U.S. Preventive Services Task Force. Ann Intern Med 136: 161-172, 2002

212. US Preventive Services Task Force: Aspirin for the primary prevention of cardiovascular events: recommendation and rationale. Ann Intern Med 136:157160,2002

213. Antithrombotic Trialists Collaboration: Collaborative meta-analysis of randomised trials of antiplatelet therapy for prevention of death, myocardial infarction, and stroke in high risk patients. BMJ 324:71-86, 2002

214. Smith SC Jr, Allen J, Blair SN, Bonow RO, Brass LM, Fonarow GC, Grundy SM, Hiratzka L, Jones D, Krumholz HM, Mosca L, Pasternak RC, Pearson T, Pfeffer MA, Taubert KA: AHA/ACC guidelines for secondary prevention for patients with coronary and other atherosclerotic vascular disease: 2006 update: endorsed by the National Heart, Lung, and Blood Institute. Circulation 113: 2363-2372, 2006

215. Campbell CL, Smyth S, Montalescot G, Steinhubl SR: Aspirin dose for the prevention of cardiovascular disease: a systematic review. JAMA 297:2018-2024, 2007

216. Sacco M, Pellegrini F, Roncaglioni MC, Avanzini F, Tognoni G, Nicolucci A: Primary prevention of cardiovascular events with low-dose aspirin and vitamin E in type 2 diabetic patients: results of the Primary Prevention Project (PPP) trial. Diabetes Care 26:3264-3272, 2003

217. Bhatt DL, Marso SP, Hirsch AT, Ringleb PA, Hacke W, Topol EJ: Amplified benefit of clopidogrel versus aspirin in patients with diabetes mellitus. Am J Cardiol 90:625-628, 2002

218. American Diabetes Asociation: Smoking and diabetes (Position Statement). Diabetes Care 27 (Suppl. 1):S74-S75, 2004

219. US Preventive Services Task Force: Counseling to prevent tobacco use and tobacco-related diseases: recommendation statement. Rockville, MD, Agency for Healthcare Research and Quality, 2003

220. Ranney L, Melvin C, Lux L, McClain E, Lohr KN: Systematic review: smoking cessation intervention strategies for adults and adults in special populations. Ann Intern Med 145:845-856, 2006

221. Scognamiglio R, Negut C, Ramondo A, Tiengo A, Avogaro A: Detection of coronary artery disease in asymptomatic patients with type 2 diabetes mellitus. J Am Coll Cardiol 47:65-71, 2006

222. Boden WE, O'Rourke RA, Teo KK, Har- tigan PM, Maron DJ, Kostuk WJ, Knudtson M, Dada M, Casperson P, Harris CL, Chaitman BR, Shaw L, Gosselin G, Nawaz S, Title LM, Gau G, Blaustein AS, Booth DC, Bates ER, Spertus JA, Berman DS, Mancini GB, Weintraub WS: Optimal medical therapy with or without PCI for stable coronary disease. N Engl J Med 356:1503-1516, 2007

223. Wackers FJ, Chyun DA, Young LH, Heller GV, Iskandrian AE, Davey JA, Barrett EJ, Taillefer R, Wittlin SD, Filipchuk N, Ratner RE, Inzucchi SE: Resolution of asymptomatic myocardial ischemia in patients with type 2 diabetes in the Detection of Ischemia in Asymptomatic Diabetics (DIAD) study. Diabetes Care 30:2892-2898, 2007

224. Braunwald E, Domanski MJ, Fowler SE, Geller NL, Gersh BJ, Hsia J, Pfeffer MA, Rice MM, Rosenberg YD, Rouleau JL: Angiotensin-converting-enzyme inhibition in stable coronary artery disease. N Engl J Med 351:2058-2068, 2004

225. Effects of the angiotensin-receptor blocker telmisartan on cardiovascular events in high-risk patients intolerant to angiotensin-converting enzyme inhibitors: a randomised controlled trial. Lancet 372:1174-1183, 2008

226. Garg JP, Bakris GL: Microalbuminuria: marker of vascular dysfunction, risk factor for cardiovascular disease. Vasc Med 7:35-43, 2002

227. Klausen K, Borch-Johnsen K, Feldt-Rasmussen $B$, Jensen $G$, Clausen $P$, Scharling H, Appleyard M, Jensen JS: Very low levels of microalbuminuria are associated with increased risk of coronary heart disease and death independently of renal function, hypertension, and diabetes. Circulation 110:32-35, 2004

228. Gall MA, Hougaard P, Borch-Johnsen K, Parving HH: Risk factors for development of incipient and overt diabetic nephropathy in patients with non-insulin dependent diabetes mellitus: prospective, observational study. BMJ 314:783788, 1997

229. Ravid M, Lang R, Rachmani R, Lishner M: Long-term renoprotective effect of angiotensin-converting enzyme inhibition in non-insulin-dependent diabetes mellitus: a 7-year follow-up study. Arch Intern Med 156:286-289, 1996

230. Reichard P, Nilsson BY, Rosenqvist U: The effect of long-term intensified insulin treatment on the development of microvascular complications of diabetes mellitus. N Engl J Med 329:304-309, 1993

231. Effect of intensive therapy on the development and progression of diabetic nephropathy in the Diabetes Control and Complications Trial: the Diabetes Control and Complications (DCCT) Research Group. Kidney Int 47:1703- 
1720,1995

232. Lewis EJ, Hunsicker LG, Bain RP, Rohde RD: The effect of angiotensin-converting-enzyme inhibition on diabetic nephropathy: the Collaborative Study Group. N Engl J Med 329:1456-1462, 1993

233. Remuzzi G, Macia M, Ruggenenti P: Prevention and treatment of diabetic renal disease in type 2 diabetes: the BENEDICT study. J Am Soc Nephrol 17:S90S97, 2006

234. Lewis EJ, Hunsicker LG, Clarke WR, Berl T, Pohl MA, Lewis JB, Ritz E, Atkins $\mathrm{RC}$, Rohde R, Raz I: Renoprotective effect of the angiotensin-receptor antagonist irbesartan in patients with nephropathy due to type 2 diabetes. N Engl J Med 345:851-860, 2001

235. Brenner BM, Cooper ME, de Zeeuw D, Keane WF, Mitch WE, Parving HH, Remuzzi G, Snapinn SM, Zhang Z, Shahinfar S: Effects of losartan on renal and cardiovascular outcomes in patients with type 2 diabetes and nephropathy. N Engl J Med 345:861-869, 2001

236. Parving $\mathrm{HH}$, Lehnert $\mathrm{H}$, BrochnerMortensen J, Gomis R, Andersen S, Arner P: The effect of irbesartan on the development of diabetic nephropathy in patients with type 2 diabetes. $N$ Engl J Med 345:870-878, 2001

237. Pepine CJ, Handberg EM, Cooper-DeHoff RM, Marks RG, Kowey P, Messerli FH, Mancia G, Cangiano JL, Garcia-Barreto D, Keltai M, Erdine S, Bristol HA, Kolb HR, Bakris GL, Cohen JD, Parmley WW: A calcium antagonist vs a non-calcium antagonist hypertension treatment strategy for patients with coronary artery disease: the International VerapamilTrandolapril study (INVEST): a randomized controlled trial. JAMA 290:2805-2816, 2003

238. Bakris GL, Siomos M, Richardson D, Janssen I, Bolton WK, Hebert L, Agarwal R, Catanzaro D: ACE inhibition or angiotensin receptor blockade: impact on potassium in renal failure: VAL-K Study Group. Kidney Int 58:2084-2092, 2000

239. Mogensen CE, Neldam S, Tikkanen I, Oren S, Viskoper R, Watts RW, Cooper ME: Randomised controlled trial of dual blockade of renin-angiotensin system in patients with hypertension, microalbuminuria, and non-insulin dependent diabetes: the candesartan and lisinopril microalbuminuria (CALM) study. BMJ 321:1440-1444, 2000

240. Schjoedt KJ, Jacobsen P, Rossing K, Boomsma F, Parving HH: Dual blockade of the renin-angiotensin-aldosterone system in diabetic nephropathy: the role of aldosterone. Horm Metab Res 37 (Suppl. 1):4-8, 2005

241. Schjoedt KJ, Rossing K, Juhl TR, Boomsma F, Rossing P, Tarnow L, Parving $\mathrm{HH}$ : Beneficial impact of spironolac- tone in diabetic nephropathy. Kidney Int 68:2829-2836, 2005

242. Parving HH, Persson F, Lewis JB, Lewis EJ, Hollenberg NK: Aliskiren combined with losartan in type 2 diabetes and nephropathy. N Engl J Med 358:24332446, 2008

243. Pijls LT, de Vries H, Donker AJ, van Eijk JT: The effect of protein restriction on albuminuria in patients with type 2 diabetes mellitus: a randomized trial. Nephrol Dial Transplant 14:1445-1453, 1999

244. Pedrini MT, Levey AS, Lau J, Chalmers TC, Wang PH: The effect of dietary protein restriction on the progression of diabetic and nondiabetic renal diseases: a meta-analysis. Ann Intern Med 124:627632, 1996

245. Hansen HP, Tauber-Lassen E, Jensen BR, Parving HH: Effect of dietary protein restriction on prognosis in patients with diabetic nephropathy. Kidney Int 62:220-228, 2002

246. Kasiske BL, Lakatua JD, Ma JZ, Louis TA: A meta-analysis of the effects of dietary protein restriction on the rate of decline in renal function. Am J Kidney Dis 31: 954-961, 1998

247. Eknoyan G, Hostetter T, Bakris GL, Hebert L, Levey AS, Parving HH, Steffes MW, Toto R: Proteinuria and other markers of chronic kidney disease: a position statement of the national kidney foundation (NKF) and the national institute of diabetes and digestive and kidney diseases (NIDDK). Am J Kidney Dis 42: 617-622, 2003

248. Levey AS, Coresh J, Balk E, Kausz AT, Levin A, Steffes MW, Hogg RJ, Perrone RD, Lau J, Eknoyan G: National Kidney Foundation practice guidelines for chronic kidney disease: evaluation, classification, and stratification. Ann Intern Med 139:137-147, 2003

249. Kramer H, Molitch ME: Screening for kidney disease in adults with diabetes. Diabetes Care 28:1813-1816, 2005

250. Kramer HJ, Nguyen QD, Curhan G, Hsu CY: Renal insufficiency in the absence of albuminuria and retinopathy among adults with type 2 diabetes mellitus. JAMA 289:3273-3277, 2003

251. Tsalamandris C, Allen TJ, Gilbert RE, Sinha A, Panagiotopoulos S, Cooper ME, Jerums G: Progressive decline in renal function in diabetic patients with and without albuminuria. Diabetes 43:649_ 655, 1994

252. Levey AS, Bosch JP, Lewis JB, Greene T, Rogers N, Roth D: A more accurate method to estimate glomerular filtration rate from serum creatinine: a new prediction equation: Modification of Diet in Renal Disease Study Group. Ann Intern Med 130:461-470, 1999

253. Levinsky NG: Specialist evaluation in chronic kidney disease: too little, too late. Ann Intern Med 137:542-543, 2002
254. American Diabetes Association: Nephropathy in diabetes (Position Statement). Diabetes Care 27 (Suppl. 1):S79S83, 2004

255. Klein R: Hyperglycemia and microvascular and macrovascular disease in diabetes. Diabetes Care 18:258-268, 1995

256. Estacio RO, McFarling E, Biggerstaff S, Jeffers BW, Johnson D, Schrier RW: Overt albuminuria predicts diabetic retinopathy in Hispanics with NIDDM. Am J Kidney Dis 31:947-953, 1998

257. Leske MC, Wu SY, Hennis A, Hyman L, Nemesure B, Yang L, Schachat AP: Hyperglycemia, blood pressure, and the 9-year incidence of diabetic retinopathy: the Barbados Eye Studies. Ophthalmology 112:799-805, 2005

258. Fong DS, Aiello LP, Ferris FL III, Klein R: Diabetic retinopathy. Diabetes Care 27: 2540-2553, 2004

259. DCCT: Effect of pregnancy on microvascular complications in the diabetes control and complications trial: The Diabetes Control and Complications Trial Research Group. Diabetes Care 23: 1084-1091, 2000

260. The Diabetic Retinopathy Study (DRS) Research Group: Preliminary report on the effects of photocoagulation therapy: DRS Report \#1. Am J Ophthalmol 81: 383-396, 1976

261. Photocoagulation for diabetic macular edema: Early Treatment Diabetic Retinopathy Study report number 1: Early Treatment Diabetic Retinopathy Study Research Group. Arch Ophthalmol 103: 1796-1806, 1985

262. Klein R, Klein BE, Moss SE, Davis MD, DeMets DL: The Wisconsin epidemiologic study of diabetic retinopathy. II. Prevalence and risk of diabetic retinopathy when age at diagnosis is less than 30 years. Arch Ophthalmol 102:520-526, 1984

263. Harris MI, Klein R, Welborn TA, Knuiman MW: Onset of NIDDM occurs at least 4-7 yr before clinical diagnosis. Diabetes Care 15:815-819, 1992

264. Vijan S, Hofer TP, Hayward RA: Costutility analysis of screening intervals for diabetic retinopathy in patients with type 2 diabetes mellitus. JAMA 283:889-896, 2000

265. Klein R: Screening interval for retinopathy in type 2 diabetes. Lancet 361:190191, 2003

266. Younis N, Broadbent DM, Vora JP, Harding SP: Incidence of sight-threatening retinopathy in patients with type 2 diabetes in the Liverpool Diabetic Eye Study: a cohort study. Lancet 361:195200, 2003

267. Ahmed J, Ward TP, Bursell SE, Aiello LM, Cavallerano JD, Vigersky RA: The sensitivity and specificity of nonmydriatic digital stereoscopic retinal imaging in detecting diabetic retinopathy. Diabe- 
tes Care 29:2205-2209, 2006

268. American Diabetes Association: Retinopathy in diabetes. Diabetes Care 27 (Suppl. 1):S84-S87, 2004

269. Ciulla TA, Amador AG, Zinman B: Diabetic retinopathy and diabetic macular edema: pathophysiology, screening, and novel therapies. Diabetes Care 26:26532664, 2003

270. Boulton AJ, Vinik AI, Arezzo JC, Bril V, Feldman EL, Freeman R, Malik RA, Maser RE, Sosenko JM, Ziegler D: Diabetic neuropathies: a statement by the American Diabetes Association. Diabetes Care 28:956-962, 2005

271. Vinik AI, Maser RE, Mitchell BD, Freeman R: Diabetic autonomic neuropathy. Diabetes Care 26:1553-1579, 2003

272. Boulton AJ, Armstrong DG, Albert SF, Frykberg RG, Hellman R, Kirkman MS, Lavery LA, Lemaster JW, Mills JL Sr, Mueller MJ, Sheehan P, Wukich DK: Comprehensive foot examination and risk assessment: a report of the task force of the foot care interest group of the American Diabetes Association, with endorsement by the American Association of Clinical Endocrinologists. Diabetes Care 31:1679-1685, 2008

273. American Diabetes Association: Peripheral arterial disease in people with diabetes (Consensus Statement). Diabetes Care 26:3333-3341, 2003

274. American Diabetes Association: Consensus Development Conference on Diabetic Foot Wound Care: 7-8 April 1999, Boston, Massachusetts. Diabetes Care 22:1354-1360, 1999

275. Silverstein J, Klingensmith G, Copeland KC, Plotnick L, Kaufman F, Laffel L, Deeb LC, Grey M, Anderson BJ, Holzmeister LA, Clark NG: Care of children and adolescents with type 1 diabetes mellitus: a statement of the American Diabetes Association. Diabetes Care 28: $186-212,2005$

276. Northam EA, Anderson PJ, Werther GA, Warne GL, Adler RG, Andrewes D: Neuropsychological complications of IDDM in children 2 years after disease onset. Diabetes Care 21:379-384, 1998

277. Rovet J, Alvarez M: Attentional functioning in children and adolescents with IDDM. Diabetes Care 20:803-810, 1997

278. Bjorgaas M, Gimse R, Vik T, Sand T: Cognitive function in type 1 diabetic children with and without episodes of severe hypoglycaemia. Acta Paediatr 86: 148-153, 1997

279. Doyle EA, Weinzimer SA, Steffen AT, Ahern JA, Vincent M, Tamborlane WV: A randomized, prospective trial comparing the efficacy of continuous subcutaneous insulin infusion with multiple daily injections using insulin glargine. Diabetes Care 27:1554-1558, 2004

280. Nimri R, Weintrob $N$, Benzaquen $H$, Ofan R, Fayman G, Phillip M: Insulin pump therapy in youth with type 1 diabetes: a retrospective paired study. Pediatrics 117:2126-2131, 2006

281. Krantz JS, Mack WJ, Hodis HN, Liu CR, Liu CH, Kaufman FR: Early onset of subclinical atherosclerosis in young persons with type 1 diabetes. J Pediatr 145:452457, 2004

282. Jarvisalo MJ, Putto-Laurila A, Jartti L, Lehtimaki T, Solakivi T, Ronnemaa T, Raitakari OT: Carotid artery intima-media thickness in children with type 1 diabetes. Diabetes 51:493-498, 2002

283. Haller MJ, Samyn M, Nichols WW, Brusko T, Wasserfall C, Schwartz RF, Atkinson M, Shuster JJ, Pierce GL, Silverstein JH: Radial artery tonometry demonstrates arterial stiffness in children with type 1 diabetes. Diabetes Care 27:2911-2917, 2004

284. Orchard TJ, Forrest KY, Kuller LH, Becker DJ: Lipid and blood pressure treatment goals for type 1 diabetes: 10year incidence data from the Pittsburgh Epidemiology of Diabetes Complications Study. Diabetes Care 24:10531059,2001

285. Kavey RE, Allada V, Daniels SR, Hayman LL, McCrindle BW, Newburger JW, Parekh RS, Steinberger J: Cardiovascular risk reduction in high-risk pediatric patients: a scientific statement from the American Heart Association Expert Panel on Population and Prevention Science; the Councils on Cardiovascular Disease in the Young, Epidemiology and Prevention, Nutrition, Physical Activity and Metabolism, High Blood Pressure Research, Cardiovascular Nursing, and the Kidney in Heart Disease; and the Interdisciplinary Working Group on Quality of Care and Outcomes Research: endorsed by the American Academy of Pediatrics. Circulation 114:2710-2738, 2006

286. McCrindle BW, Urbina EM, Dennison BA, Jacobson MS, Steinberger J, Rocchini AP, Hayman LL, Daniels SR: Drug therapy of high-risk lipid abnormalities in children and adolescents: a scientific statement from the American Heart Association Atherosclerosis, Hypertension, and Obesity in Youth Committee, Council of Cardiovascular Disease in the Young, with the Council on Cardiovascular Nursing. Circulation 115:19481967, 2007

287. Salo P, Viikari J, Hamalainen M, Lapinleimu H, Routi T, Ronnemaa T, Seppanen R, Jokinen E, Valimaki I, Simell $\mathrm{O}$ : Serum cholesterol ester fatty acids in 7- and 13-month-old children in a prospective randomized trial of a low-saturated fat, low-cholesterol diet: the STRIP baby project: Special Turku coronary Risk factor Intervention Project for children. Acta Paediatr 88:505-512, 1999

288. Efficacy and safety of lowering dietary intake of fat and cholesterol in children with elevated low-density lipoprotein cholesterol: the Dietary Intervention Study in Children (DISC): the Writing Group for the DISC Collaborative Research Group. JAMA 273:1429-1435, 1995

289. McCrindle BW, Ose L, Marais AD: Efficacy and safety of atorvastatin in children and adolescents with familial hypercholesterolemia or severe hyperlipidemia: a multicenter, randomized, placebo-controlled trial. J Pediatr 143:74-80, 2003

290. de Jongh S, Lilien MR, op't RJ, Stroes ES, Bakker HD, Kastelein JJ: Early statin therapy restores endothelial function in children with familial hypercholesterolemia. J Am Coll Cardiol 40:2117-2121, 2002

291. Wiegman A, Hutten BA, de Groot E, Rodenburg J, Bakker HD, Buller HR, Sijbrands EJ, Kastelein JJ: Efficacy and safety of statin therapy in children with familial hypercholesterolemia: a randomized controlled trial. JAMA 292: 331-337, 2004

292. Holmes GK: Screening for coeliac disease in type 1 diabetes. Arch Dis Child 87:495-498, 2002

293. Rewers M, Liu E, Simmons J, Redondo MJ, Hoffenberg EJ: Celiac disease associated with type 1 diabetes mellitus. Endocrinol Metab Clin North Am 33:197214, 2004

294. Roldan MB, Alonso M, Barrio R: Thyroid autoimmunity in children and adolescents with type 1 diabetes mellitus. Diabetes Nutr Metab 12:27-31, 1999

295. Kordonouri O, Deiss D, Danne T, Dorow A, Bassir C, Gruters-Kieslich A: Predictivity of thyroid autoantibodies for the development of thyroid disorders in children and adolescents with type 1 diabetes. Diabet Med 19:518-521, 2002

296. Mohn A, Di Michele S, Di Luzio R, Tumini S, Chiarelli F: The effect of subclinical hypothyroidism on metabolic control in children and adolescents with type 1 diabetes mellitus. Diabet Med 19: 70-73, 2002

297. Chase HP, Garg SK, Cockerham RS, Wilcox WD, Walravens PA: Thyroid hormone replacement and growth of children with subclinical hypothyroidism and diabetes. Diabet Med 7:299-303, 1990

298. Eppens MC, Craig ME, Cusumano J, Hing S, Chan AK, Howard NJ, Silink M, Donaghue KC: Prevalence of diabetes complications in adolescents with type 2 compared with type 1 diabetes. Diabetes Care 29:1300-1306, 2006

299. Kitzmiller JL, Gavin LA, Gin GD, Jovanovic-Peterson L, Main EK, Zigrang WD: Preconception care of diabetes: glycemic control prevents congenital anomalies. JAMA 265:731-736, 1991 
300. Goldman JA, Dicker D, Feldberg D, Yeshaya A, Samuel N, Karp M: Pregnancy outcome in patients with insulin-dependent diabetes mellitus with preconceptional diabetic control: a comparative study. AmJ Obstet Gynecol 155:293-297, 1986

301. Rosenn B, Miodovnik M, Combs CA, Khoury J, Siddiqi TA: Pre-conception management of insulin-dependent diabetes: improvement of pregnancy outcome. Obstet Gynecol 77:846-849, 1991

302. Tchobroutsky C, Vray MM, Altman JJ: Risk/benefit ratio of changing late obstetrical strategies in the management of insulin-dependent diabetic pregnancies: a comparison between 1971-1977 and 1978-1985 periods in 389 pregnancies. Diabete Metab 17:287-294, 1991

303. Willhoite MB, Bennert HW Jr, Palomaki GE, Zaremba MM, Herman WH, Williams JR, Spear NH: The impact of preconception counseling on pregnancy outcomes: the experience of the Maine Diabetes in Pregnancy Program. Diabetes Care 16:450-455, 1993

304. Cooper WO, Hernandez-Diaz S, Arbogast PG, Dudley JA, Dyer S, Gideon PS, Hall K, Ray WA: Major congenital malformations after first-trimester exposure to ACE inhibitors. $N$ Engl $J$ Med 354:2443-2451, 2006

305. Kitzmiller JL, Buchanan TA, Kjos S, Combs CA, Ratner RE: Pre-conception care of diabetes, congenital malformations, and spontaneous abortions. Diabetes Care 19:514-541, 1996

306. American Diabetes Association: Preconception care of women with diabetes (Position Statement). Diabetes Care 27 (Suppl. 1):S76-S78, 2004

307. Brown AF, Mangione CM, Saliba D, Sarkisian CA: Guidelines for improving the care of the older person with diabetes mellitus. J Am Geriatr Soc 51:S265S280, 2003

308. Curb JD, Pressel SL, Cutler JA, Savage PJ, Applegate WB, Black H, Camel G, Davis BR, Frost PH, Gonzalez N, Guthrie G, Oberman A, Rutan GH, Stamler J: Effect of diuretic-based antihypertensive treatment on cardiovascular disease risk in older diabetic patients with isolated systolic hypertension: Systolic Hypertension in the Elderly Program Cooperative Research Group. JAMA 276:18861892, 1996

309. Beckett NS, Peters R, Fletcher AE, Staessen JA, Liu L, Dumitrascu D, Stoyanovsky V, Antikainen RL, Nikitin Y, Anderson C, Belhani A, Forette F, Rajkumar C, Thijs L, Banya W, Bulpitt CJ: Treatment of hypertension in patients 80 years of age or older. $N$ Engl J Med 358:1887-1898, 2008

310. Clement S, Braithwaite SS, Magee MF, Ahmann A, Smith EP, Schafer RG, Hirsh
IB: Management of diabetes and hyperglycemia in hospitals. Diabetes Care 27: 553-591, 2004

311. American Association of Clinical Endocrinologists: Inpatient diabetes and metabolic control: conference proceedings. Endocr Pract 10 (Suppl. 2):1-108, 2004

312. Garber AJ, Moghissi ES, Bransome ED Jr, Clark NG, Clement S, Cobin RH, Furnary AP, Hirsch IB, Levy P, Roberts R, van den BG, Zamudio V: American College of Endocrinology position statement on inpatient diabetes and metabolic control. EndocrPract 10 (Suppl. 2):4-9, 2004

313. ACE/ADA Task Force on Inpatient Diabetes: American College of Endocrinology and American Diabetes Association Consensus statement on inpatient diabetes and glycemic control: a call to action. Diabetes Care 29:1955-1962, 2006

314. Centers for Disease Control and Prevention: Hospitalizations for Diabetes as Any-Listed Diagnosis. National Diabetes Surveillance System. Atlanta, Georgia, Centers for Disease Control and Prevention, 2003

315. Pomposelli JJ, Baxter JK III, Babineau TJ, Pomfret EA, Driscoll DF, Forse RA, Bistrian BR: Early postoperative glucose control predicts nosocomial infection rate in diabetic patients. $J$ Parenter Enteral Nutr 22:77-81, 1998

316. Umpierrez GE, Isaacs SD, Bazargan N, You X, Thaler LM, Kitabchi AE: Hyperglycemia: an independent marker of inhospital mortality in patients with undiagnosed diabetes. J Clin Endocrinol Metab 87:978-982, 2002

317. Capes SE, Hunt D, Malmberg K, Gerstein HC: Stress hyperglycaemia and increased risk of death after myocardial infarction in patients with and without diabetes: a systematic overview. Lancet 355:773-778, 2000

318. Bolk J, van der PT, Cornel JH, Arnold AE, Sepers J, Umans VA: Impaired glucose metabolism predicts mortality after a myocardial infarction. Int J Cardiol 79: 207-214, 2001

319. Malmberg K: Prospective randomised study of intensive insulin treatment on long term survival after acute myocardial infarction in patients with diabetes mellitus: DIGAMI (Diabetes Mellitus, Insulin Glucose Infusion in Acute Myocardial Infarction) Study Group. BMJ 314:1512-1515, 1997

320. Malmberg K, Ryden L, Efendic S, Herlitz J, Nicol P, Waldenstrom A, Wedel H, Welin L: Randomized trial of insulinglucose infusion followed by subcutaneous insulin treatment in diabetic patients with acute myocardial infarction (DIGAMI study): effects on mortality at 1 year. J Am Coll Cardiol 26:57-65, 1995

321. Malmberg K, Ryden L, Wedel H, Birke- land K, Bootsma A, Dickstein K, Efendic S, Fisher M, Hamsten A, Herlitz J, Hildebrandt P, MacLeod K, Laakso M, TorpPedersen C, Waldenstrom A: Intense metabolic control by means of insulin in patients with diabetes mellitus and acute myocardial infarction (DIGAMI 2): effects on mortality and morbidity. Eur Heart J 26:650-661, 2005

322. Mehta SR, Yusuf S, Diaz R, Zhu J, Pais P, Xavier D, Paolasso E, Ahmed R, Xie C, Kazmi K, Tai J, Orlandini A, Pogue J, Liu L: Effect of glucose-insulin-potassium infusion on mortality in patients with acute ST-segment elevation myocardial infarction: the CREATE-ECLA randomized controlled trial. JAMA 293: 437-446, 2005

323. Cheung NW, Wong VW, McLean M: The hyperglycemia: intensive insulin infusion in infarction (HI-5) study: a randomized controlled trial of insulin infusion therapy for myocardial infarction. Diabetes Care 29:765-770, 2006

324. Furnary AP, Gao G, Grunkemeier GL, $\mathrm{Wu}$ Y, Zerr KJ, Bookin SO, Floten HS, Starr A: Continuous insulin infusion reduces mortality in patients with diabetes undergoing coronary artery bypass grafting. J Thorac Cardiovasc Surg 125: 1007-1021, 2003

325. Furnary AP, Zerr KJ, Grunkemeier GL, Starr A: Continuous intravenous insulin infusion reduces the incidence of deep sternal wound infection in diabetic patients after cardiac surgical procedures. Ann Thorac Surg 67:352-360, 1999

326. Golden SH, Peart-Vigilance C, Kao WH, Brancati FL: Perioperative glycemic control and the risk of infectious complications in a cohort of adults with diabetes. Diabetes Care 22:1408-1414, 1999

327. Zerr KJ, Furnary AP, Grunkemeier GL, Bookin S, Kanhere V, Starr A: Glucose control lowers the risk of wound infection in diabetics after open heart operations. Ann Thorac Surg 63:356-361, 1997

328. van den BG, Wouters P, Weekers F, Verwaest C, Bruyninckx F, Schetz M, Vlasselaers D, Ferdinande P, Lauwers P, Bouillon R: Intensive insulin therapy in the critically ill patients. N Engl J Med 345:1359-1367, 2001

329. van den BG, Wouters PJ, Bouillon R, Weekers F, Verwaest C, Schetz M, Vlasselaers D, Ferdinande P, Lauwers P: Outcome benefit of intensive insulin therapy in the critically ill: insulin dose versus glycemic control. Crit Care Med 31:359-366, 2003

330. van den $B G$, Wilmer A, Hermans $G$, Meersseman W, Wouters PJ, Milants I, Van Wijngaerden E, Bobbaers H, Bouillon R: Intensive insulin therapy in the medical ICU. N Engl J Med 354:449_ 461, 2006

331. Brunkhorst FM, Engel C, Bloos F, Meier- 
Hellmann A, Ragaller M, Weiler N, Moerer O, Gruendling M, Oppert M, Grond S, Olthoff D, Jaschinski U, John S, Rossaint R, Welte T, Schaefer M, Kern P, Kuhnt E, Kiehntopf M, Hartog C, Natanson C, Loeffler M, Reinhart K: Intensive insulin therapy and pentastarch resuscitation in severe sepsis. $N$ Engl J Med 358:125-139, 2008

332. Pittas AG, Siegel RD, Lau J: Insulin therapy for critically ill hospitalized patients: a meta-analysis of randomized controlled trials. Arch Intern Med 164:20052011, 2004

333. Wiener RS, Wiener DC, Larson RJ: Benefits and risks of tight glucose control in critically ill adults: a meta-analysis. JAMA 300:933-944, 2008

334. Krinsley J: Glycemic control in critically ill patients: Leuven and beyond. Chest 132:1-2, 2007

335. Miller CD, Phillips LS, Ziemer DC, Gallina DL, Cook CB, El Kebbi IM: Hypoglycemia in patients with type 2 diabetes mellitus. Arch Intern Med 161: 1653-1659, 2001

336. Misbin RI, Green L, Stadel BV, Gueriguian JL, Gubbi A, Fleming GA: Lactic acidosis in patients with diabetes treated with metformin. N Engl J Med 338:265266, 1998

337. Misbin RI: The phantom of lactic acidosis due to metformin in patients with diabetes. Diabetes Care 27:1791-1793, 2004

338. Salpeter SR, Greyber E, Pasternak GA, Salpeter EE: Risk of fatal and nonfatal lactic acidosis with metformin use in type 2 diabetes mellitus: systematic review and meta-analysis. Arch Intern Med 163:2594-2602, 2003

339. Queale WS, Seidler AJ, Brancati FL: Glycemic control and sliding scale insulin use in medical inpatients with diabetes mellitus. Arch Intern Med 157:545-552, 1997

340. Gearhart JG, Duncan JL, III, Replogle WH, Forbes RC, Walley EJ: Efficacy of sliding-scale insulin therapy: a comparison with prospective regimens. Fam Pract Res J 14:313-322, 1994

341. Walts LF, Miller J, Davidson MB, Brown $\mathrm{J}$ : Perioperative management of diabetes mellitus. Anesthesiology 55:104-109, 1981

342. Umpierrez GE, Smiley D, Zisman A, Prieto LM, Palacio A, Ceron M, Puig A, Mejia R: Randomized study of basal-bolus insulin therapy in the inpatient management of patients with type 2 diabetes (RABBIT 2 trial). Diabetes Care 30: 2181-2186, 2007

343. Schmeltz LR, DeSantis AJ, Schmidt K, O'Shea-Mahler E, Rhee C, Brandt S, Peterson S, Molitch ME: Conversion of intravenous insulin infusions to subcutaneously administered insulin glargine in patients with hyperglycemia. Endocr
Pract 12:641-650, 2006

344. Shilo S, Berezovsky S, Friedlander Y, Sonnenblick M: Hypoglycemia in hospitalized nondiabetic older patients. J Am Geriatr Soc 46:978-982, 1998

345. Fischer KF, Lees JA, Newman JH: Hypoglycemia in hospitalized patients: causes and outcomes. N Engl J Med 315:12451250, 1986

346. Markovitz LJ, Wiechmann RJ, Harris N, Hayden V, Cooper J, Johnson G, Harelstad R, Calkins L, Braithwaite SS: Description and evaluation of a glycemic management protocol for patients with diabetes undergoing heart surgery. EndocrPract 8:10-18, 2002

347. Levetan CS, Salas JR, Wilets IF, Zumoff B: Impact of endocrine and diabetes team consultation on hospital length of stay for patients with diabetes. Am J Med 99:22-28, 1995

348. Levetan CS, Passaro MD, Jablonski KA, Ratner RE: Effect of physician specialty on outcomes in diabetic ketoacidosis. Diabetes Care 22:1790-1795, 1999

349. Koproski J, Pretto Z, Poretsky L: Effects of an intervention by a diabetes team in hospitalized patients with diabetes. Diabetes Care 20:1553-1555, 1997

350. Furnary AP, Braithwaite SS: Effects of outcome on in-hospital transition from intravenous insulin infusion to subcutaneous therapy. Am J Cardiol 98:557564, 2006

351. American Diabetes Association: Diabetes nutrition recommendations for health care institutions (Position Statement). Diabetes Care 27 (Suppl. 1):S55S57, 2004

352. Boucher JL, Swift CS, Franz MJ, Kulkarni K, Schafer RG, Pritchett E, Clark NG: Inpatient management of diabetes and hyperglycemia: implications for nutrition practice and the food and nutrition professional. J Am Diet Assoc 107:105-111, 2007

353. American Diabetes Association: Diabetes care in the school and day care setting. Diabetes Care 29 (Suppl. 1):S49S55, 2006

354. National Diabetes Education Program: Helping the student with diabetes succeed: a guide for school personnel (article online), 2006. Available from http:// www.ndep.nih.gov/resources/school.htm

355. American Diabetes Association: Diabetes care tasks at school: what key personnel need to know (article online), 2005. Available from http://diabetes.org

356. American Diabetes Association: Diabetes care at diabetes camps. Diabetes Care 29 (Suppl. 1):S56-S58, 2006

357. American Diabetes Association: Diabetes management in correctional institutions. Diabetes Care 29 (Suppl. 1):S59S66, 2006
358. American Diabetes Association Statement on Emergency and Disaster Preparedness: a report of the Disaster Response Task Force. Diabetes Care 30: 2395-2398, 2007

359. Cefalu WT, Smith SR, Blonde L, Fonseca $\mathrm{V}$ : The Hurricane Katrina aftermath and its impact on diabetes care: observations from "ground zero": lessons in disaster preparedness of people with diabetes. Diabetes Care 29:158-160, 2006

360. American Diabetes Association: Hypoglycemia and employment/licensure. Diabetes Care 29 (Suppl. 1):S67, 2006

361. American Diabetes Association: Thirdparty reimbursement for diabetes care, self-management education, and supplies. Diabetes Care 29 (Suppl. 1):S68S69, 2006

362. Saydah SH, Fradkin J, Cowie CC: Poor control of risk factors for vascular disease among adults with previously diagnosed diabetes. JAMA 291:335-342, 2004

363. Clark CM Jr, Snyder JW, Meek RL, Stutz LM, Parkin CG: A systematic approach to risk stratification and intervention within a managed care environment improves diabetes outcomes and patient satisfaction. Diabetes Care 24:10791086, 2001

364. Meigs JB, Cagliero E, Dubey A, MurphySheehy P, Gildesgame C, Chueh H, Barry MJ, Singer DE, Nathan DM: A controlled trial of web-based diabetes disease management: the MGH diabetes primary care improvement project. Diabetes Care 26:750-757, 2003

365. O'Connor PJ, Desai J, Solberg LI, Reger LA, Crain AL, Asche SE, Pearson TL, Clark CK, Rush WA, Cherney LM, Sperl-Hillen JM, Bishop DB: Randomized trial of quality improvement intervention to improve diabetes care in primary care settings. Diabetes Care 28: 1890-1897, 2005

366. Sperl-Hillen JM, O'Connor PJ: Factors driving diabetes care improvement in a large medical group: ten years of progress. Am J Manag Care 11:S177S185, 2005

367. Siminerio LM: Implementing diabetes self-management training programs: breaking through the barriers in primary care. Endocr Pract 12 (Suppl. 1):124130, 2006

368. Mahoney JJ: Reducing patient drug acquisition costs can lower diabetes health claims. Am J Manag Care 11:S170_S176, 2005

369. Maney M, Tseng CL, Safford MM, Miller DR, Pogach LM: Impact of self-reported patient characteristics upon assessment of glycemic control in the Veterans Health Administration. Diabetes Care 30:245-251, 2007 
370. Bergenstal RM: Treatment models from the International Diabetes Center: advancing from oral agents to insulin therapy in type 2 diabetes. Endocr Pract 12 (Suppl. 1):98-104, 2006
371. O'Connor PJ: Electronic medical records and diabetes care improvement: are we waiting for Godot? Diabetes Care 26:942-943, 2003

372. Shojania KG, Ranji SR, McDonald KM,
Grimshaw JM, Sundaram V, Rushakoff RJ, Owens DK: Effects of quality improvement strategies for type 2 diabetes on glycemic control: a meta-regression analysis. JAMA 296:427-440, 2006 